Newton Mayer Solórzano Chávez

Métricas de Finsler Esfericamente Simétricas

Brasília

2015 
Newton Mayer Solórzano Chávez

\title{
Métricas de Finsler Esfericamente Simétricas
}

Tese apresentada ao Departamento de Matemática da Universidade de Brasília, como parte dos requisitos para obtenção do grau de DOUTOR EM MATEMÁTICA

\author{
Universidade de Brasília - UNB \\ Instituto de Ciências Exatas \\ Departamento de Matemática \\ Programa de Pós-Graduação
}

Orientadora: Keti Tenenblat

Brasília

2015 
Ficha catalográfica elaborada pela Biblioteca Central da Universidade de Brasília. Acervo 1020784.

\footnotetext{
Solórzano Chávez, Newton Mayer.

Métricas de Finsler esfericamente simétricas / Newton Mayer Solórzano Chávez. - 2015.

i i, $77 ; 30 \mathrm{~cm}$.

Tese (doutorado) - Universidade de Brasília, Instituto de Ciências Exatas, Departamento de Matemática, Programa de Pós-Graduação em Matemática, 2015.

Inclui bibliografia.

Orientação: Keti Tenenblat.

1. Finsler, Paul, 1894-1970. 2. Geometria diferencial. I. Tenenblat, Keti. II. Título.
}

CDU 514.763 .62 
Este trabalho é dedicado aos meus pais

CAYETANO SOLÓRZANO e OLIMPIA CHÁVEZ. 


\section{AGRADECIMENTOS}

É conhecido que o homem é de natureza sociável, é por isso, que o homem estendeuse notavelmente no âmbito do científico. Quero agradecer então, as pessoas que tem influenciado na elaboração de este trabalho.

Em primeiro lugar quero agradecer a minha orientadora, a professora Dra. Keti Tenenblat, não encontro palavras suficientes para expressar minha gratidão e admiração. Quero agradecer por tudo o que representa na minha vida, sempre foi uma fonte de conhecimento e conselhos, pois de acordo a seus conhecimentos e experiências fez do processo de execução de este trabalho, uma experiência favorável na minha formação profissional, e no âmbito pessoal sempre se disponibilizou para escutar e aconselhar.

Agradeço em segundo lugar aqueles que são o suporte de meus ideais e paixões: meus pais, que sempre apoiaram minhas decisões no material e por suposto no espiritual. Faltam palavras para agradecer-os por tudo o que representam na minha vida. Imagino minha vida sem pais assim, e no vejo um futuro bom.

À todos eles devo minhas bases na Matemática.

Em terceiro lugar, agradeço a meus amigos, companheiros de aulas (de guerra), que de uma ou outra forma influenciaram na execução deste trabalho.

Em quarto lugar, quero agradecer à meus professores mais influentes no aspecto do meu processo de evolução mental e espiritual, no ensino médio, na universidade do Perú, na UFG (em especial ao meu orientador de mestrado: Prof. Marcelo) e na UNB. A vocês devo meu avanço na Matemática e o carinho pela Geometria.

E por último, agraço a CAPES e CNPQ pelo apoio financeiro, que é de muita importância.

Gracias a todos ellos, no los olvidaré.

(Obrigado, não esquecerei de vocês.) 
"É provável que viver na terra seja caro, mas inclui uma viagem gratuita em torno do Sol a cada ano. -Adágio Popular 


\section{RESUMO}

Consideramos métricas de Finsler esfericamente simétricas do tipo Douglas. Caracterizamos tais métricas por uma equação diferencial e obtemos a solução geral desta equação em termos de quatro funções arbitrárias. Quando as métricas de Finsler são esfericamente simétricas mostramos que as métricas do tipo Berwald coincidem com as do tipo Landsberg. Provamos que o problema de classificar as métricas esfericamente simétricas do tipo Douglas com $S$-curvatura nula reduz-se a classificar as métricas esfericmanete simétricas do tipo Berwald ou Landsberg. Obtemos a classificação de tais métricas. Incluímos vários exemplos e classes de novas métricas de Douglas.

Palavras-chaves: Finsler. Esfericamente simétrica. Curvatura de Douglas. Curvatura de Landsberg. Curvatura de Berwald. 


\section{ABSTRACT}

We consider spherically symmetric Finsler metrics of Douglas type. We characterize such metrics by a differential equation and we obtain the general solution of this equation in terms of four arbitrary functions. For spherically symmetric Finsler metrics we show that the metrics of Berwald type coincide whit those of Landsberg type. We prove that the problem of classifying the spherically symmetric Douglas metrics whose $S$-curvature vanishes reduce to classifying the spherical symmetric metrics of Berwald or Landsberg type. We obtain the classification of such metrics. We include several examples and new classes of Douglas metrics.

Key-words: Finsler. Spherically symmetric. Douglas Curvature. Landsberg Curvature. Berwald curvature. 


\section{SUMÁRIO}

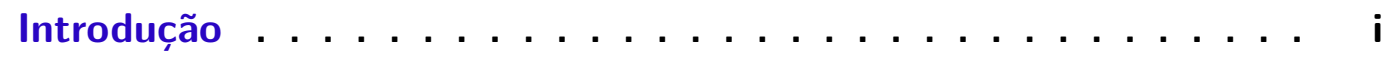

$1 \quad$ Preliminares $\ldots \ldots \ldots \ldots \ldots \ldots$

$1.1 \quad$ Métricas de Finsler . . . . . . . . . . . . . . . . 1

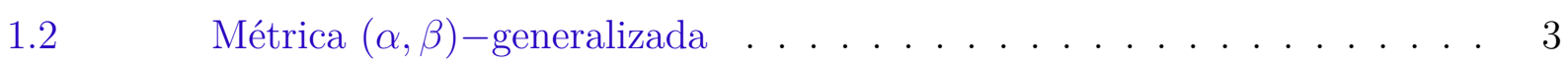

1.3 Geodésicas e Campos de Vetores Paralelos . . . . . . . . . . . . 5

1.4 Métricas de Berwald e Landsberg . . . . . . . . . . . . . . . 9

$1.5 \quad$ Métricas de Douglas . . . . . . . . . . . . . . 13

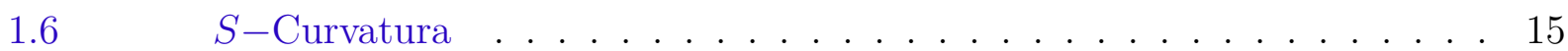

$1.7 \quad$ Métricas esfericamente Simétricas . . . . . . . . . . . . . . . . 21

Equação de Transporte . . . . . . . . . . . . . . . 26

$2 \quad$ Métricas de Douglas . . . . . . . . . . . . . . . . . 31

$2.1 \quad$ Métricas de Douglas Esfericamente Simétricas . . . . . . . . . . . . 31

2.2 Caracterização das Métricas de Douglas Esfericamente Simétricas . . . . 40

2.3 Exemplos de Famílias de Métricas de Douglas . . . . . . . . . . . . . . 44

3 Métricas de Berwald e métricas de Landsberg . . . . . . . . . . . 49

$3.1 \quad$ Métricas de Berwald Esfericamente Simétricas . . . . . . . . . . . . . 49

3.2 Métricas de Landsberg Esfericamente Simétricas . . . . . . . . . . . . 51

4 Métricas de Douglas com S-curvatura nula . . . . . . . . . . . 61

$4.1 \quad$ Métricas de Douglas esfericamente simétrica com S-curvatura nula . . . 61

4.2 Caracterização em termos de Métricas de Berwald e de Landsberg . . . 63

Referências . . . . . . . . . . . . . . . . . 74 


\section{INTRODUÇÃO}

Podemos dizer que o que se conhece hoje por Geometria de Finsler tem suas origens nos trabalhos de Bernhard Riemann a partir de 1854, porém seu nome se deve ao matemático alemão Paul Finsler (1894-1970), que estudou diversos fundamentos dessa geometria em cálculo das variações, publicando sua tese em 1918. Variedades de Finsler generalizam a noção de variedades Riemannianas, onde a norma de uma forma quadrática positiva definida é substituída por uma norma com propriedades mais fracas, a chamada norma de Minkowski. Modelos dinâmicos descritos pela Geometria de Finsler aparecem naturalmente em diversas áreas como mecânica clássica, ótica geométrica, mecânica quântica, etc. A partir de Finsler, diversos matemáticos tiveram importância central para o desenvolvimento desta teoria no século XX, como L. Berwald, E. Cartan, S-S. Chern e outros.

No Capítulo 1, incluímos uma breve introdução à Geometria de Finsler e estudamos certas classes destas métricas como as $(\alpha, \beta)$-métricas generalizadas que foram introduzidas por C. Yu e H. Zhu em [40]. Introduzimos também certas curvaturas como a curvatura de Berwald, curvatura de Landsberg, curvatura de Douglas e a $S$-curvatura. As métricas com curvatura de Berwald nula são chamadas métricas de Berwald e são uma generalização das métricas projetivamente planas (métricas cujas geodésicas são linhas de reta) e das métricas Riemannianas. As métricas com curvatura de Landsberg e Douglas nula são chamadas métricas de Landsberg e Douglas, respectivamente e são generalizações das métricas de Berwald. Neste capítulo também introduzimos as métricas esfericamente simétricas que foram estudadas pela primeira vez por Rutz em [27]. Para finalizar o Capítulo 1, estudamos o método das curvas características para solucionar equações diferenciais parciais da forma

$$
\psi_{r}(r, s)+\nu(r, s) \psi_{s}(r, s)=P(r, s, \psi(r, s)),
$$

onde $\nu(r, s)$ e $P(r, s, \psi(r, s))$ são diferenciáveis.

No Capítulo 2, estudamos as métricas esfericamente simétricas do tipo Douglas. No Teorema 2.2.1, caracterizamos tais métricas por uma equação diferencial parcial. No Teorema 2.2.2, usando o método das curvas características, obtemos a solução geral para a equação diferencial parcial. Como consequência, observamos que as métricas esfericamente simétricas do tipo Douglas são muito ricas já que dependem de 4 funções arbitrárias. Na parte final deste Capítulo, apresentamos exemplos e classes de novas métricas do tipo Douglas.

No Capítulo 3, no espírito de procurar um exemplo de métrica to tipo Landsberg que não seja do tipo Berwald, conhecido como o problema do "unicórnio", caracterizamos as métricas esfericamente simétricas do tipo Berwald e do tipo Landsberg mediante equações 
diferenciais (Teorema 3.1.3 e Proposição 3.2.2). Estudando estas equações concluímos que estes tipos de métricas são equivalentes quando a métrica de Finsler é esfericamente simétrica (Teorema 3.2.3).

No Capítulo 4, consideramos métricas esfericamente simétricas e o elemento de volume $d V_{B H}$ Busemann-Hausdorff para caracterizar tais métricas do tipo Douglas com $S$-curvatura nula mediante um sistema de equações diferenciais parciais (ver Teorema 4.1.2). Notamos que este sistema está estreitamente relacionado com o sistema dado no Teorema 3.1.3 que caracteriza métricas do tipo Berwald, provamos então o Teorema 4.1.3 que afirma: toda métrica esfericamente simétrica é do tipo Douglas com $S$-curvatura nula se, e somente se, é do tipo Berwald. Assim o problema de classificar métricas esfericamente simétricas com $S$-curvatura nula se reduz a classificar métricas esfericamente simétricas do tipo Berwald ou Landsberg (ver Teorema 3.2.3), cujo sistema de equações diferenciais parciais foi dado no capítulo anterior (Teorema 3.1.3). Mediante a técnica de curvas características dada no Capítulo 1, obtemos a classificação de tais métricas no Teorema 4.2.1.

São enunciadas consequências interessantes do Teorema 4.2.1, tais como o Corolário 4.2.1 que nos fornece o elemento de volume $d V_{B H}$, e o Corolário 4.2.2 que é consequência conjunta com o Teorema 3.2.3 que nos dá uma classificação das métricas de Landsberg (ou Berwald ou Douglas com $S$-curvatura nula) sobre $\mathbb{R}^{n}$. Incluímos algumas observações no caso em que se considera o elemento de volume $d V_{H T}$ Holmes-Thompson. E finalmente damos alguns exemplos interessantes. 


\section{PRELIMINARES}

Neste capítulo, apresentaremos algumas definições e resultados que servirão para o desenvolvimento dos capítulos subsequentes.

Faremos uso da convenção de Einstein, ou seja, não escreveremos o símbolo do somatório para representar a soma quando aparecerem índices repetidos.

\subsection{Métricas de Finsler}

Seja $V$ um espaço vetorial de dimensão $n$. Diremos que $F: V \rightarrow[0, \infty)$ é métrica de Minkowski se satisfaz as seguintes propriedades:

(M1) $F$ é $C^{\infty}$ sobre $V \backslash\{0\}$;

(M2) $F(\lambda y)=\lambda F(y)$, para todo $y \in V \operatorname{com} \lambda>0$;

(M3) para cada $y \in V \backslash\{0\}$, a forma bilinear simétrica $g_{y}$ sobre $V$ é positiva definida, onde

$$
g_{y}:=\left.\frac{1}{2} \frac{\partial^{2}}{\partial s \partial t}\left[F^{2}(y+s u+t v)\right]\right|_{s=t=0}, \quad u, v \in V .
$$

O par $(V, F)$ é chamado espaço de Minkowski.

Todo espaço vetorial de dimensão $n$ é linearmente isomorfo a $\mathbb{R}^{n}$, cujos elementos $y$ são da forma $\left(y_{1}, \ldots y_{n}\right)=y$. Então podemos considerar, sem perda de generalidade, as métricas de Minkowski sobre $\mathbb{R}^{n}$.

Seja $(V, F)$ um espaço de Minkowski. Fixemos uma base $\left\{b_{i}\right\}$ para $V$, e consideremos $F(y)=F\left(y^{i} b_{i}\right)$ como função de $\left(y^{i}\right) \in \mathbb{R}^{n}$. Então para $y \neq 0$,

$$
g_{i j}(y):=\mathbf{g}_{y}\left(b_{i}, b_{j}\right)=\frac{1}{2}\left[F^{2}\right]_{y^{i} y^{j}}(y),
$$

onde $\left[F^{2}\right]_{y^{i} y^{j}}(y)$ denota a derivada parcial de $F^{2}$ em relação a $y^{i}$ e $y^{j}$. Assim, temos

$$
g_{y}(u, v)=g_{i j}(y) u^{i} u^{j}, \quad u=u^{i} b_{i}, \quad v=v^{j} b_{j}
$$

e usando o Teorema de Euler (Teorema 1.1.1),

$$
F(y)=\sqrt{g_{i j}(y) y^{i} y^{j}}, \quad y=y^{i} b_{i}
$$

De agora em diante $M$ denotará uma variedade diferenciável de dimensão $n$. Sendo $T_{x} M$ um espaço vetorial, uma métrica de Finsler pode ser vista como uma família de normas de Minkowski sobre cada $T_{x} M$ (variando diferenciavelmente com $x \in M$ ), isto é: 
Definição 1.1.1. Sejam $M$ uma variedade diferenciável de dimensão $n$ e $T M=\cup_{x \in M} T_{x} M$ o fibrado tangente de $M$, onde $T_{x} M$ é o espaço tangente em $x \in M$. Consideremos $T M_{o}:=T M \backslash\{0\}$, onde $\{0\}$ entende-se por $\left\{(x, 0) \mid x \in M, 0 \in T_{x} M\right\}$. Diremos que uma função $F: T M \rightarrow[0, \infty)$ é uma métrica de Finsler sobre $M$ se $F$ satisfaz as seguintes propriedades:

(a) $F$ é $C^{\infty}$ sobre $T M_{o}$;

(b) em cada ponto $x \in M$, a restrição $F_{x}:=\left.F\right|_{T_{x} M}$ é uma norma de Minkowski sobre $T_{x} M$.

\section{Exemplos:}

1. (Métrica Riemanniana) Seja $g=\left\{g_{x}\right\}_{x \in M}$, onde $g_{x}$ é uma forma bilinear simétrica positiva definida sobre $T_{x} M$ tal que em coordenadas locais $\left(x^{i}\right)$,

$$
g_{i j}(x)=g_{x}\left(\left.\frac{\partial}{\partial x^{i}}\right|_{x},\left.\frac{\partial}{\partial x^{j}}\right|_{x}\right)
$$

são funções $C^{\infty}, g$ é chamada métrica Riemanniana. Defina

$$
F_{x}(y)=\sqrt{g_{x}(y, y)}, \quad y \in T_{x} M
$$

Da definição, vemos que $F_{x}$ é uma norma Euclidiana. Assim a família de normas Euclidianas $F=F_{x \in M}$ é uma métrica de Finsler sobre $M$.

Uma métrica $F$ de Finsler é chamada Riemanniana se pode ser expressa por (1.2) para alguma métrica Riemanniana $g$.

2. (Métrica de Randers) Sejam $\alpha(x, y)=\sqrt{a_{i j}(x) y^{i} y^{j}}$ uma métrica Riemanniana e $\beta(x, y)=b_{i}(x) y^{i}$ uma 1-forma definida sobre uma variedade $M$ com coordenadas locais $\left(x^{i}\right)$ e $y=y^{i} \frac{\partial}{\partial x^{i}}$. Para cada $x \in M$, assuma que

$$
\|\beta\|_{x}=\sup _{y \in T_{x} M: \alpha(y)=1} \beta(y)<1 .
$$

Então $F:=\alpha+\beta$ definida sobre $M$ é uma métrica de Finsler, chamada métrica de Randers. As métricas de Randers foram introduzidas pelo físico G. Randers, em 1941 [26].

3. (Métrica de Funk)[32] Seja $\Omega$ um domínio convexo em $\mathbb{R}^{n}$. A métrica de Funk $F$ é definida por

$$
x+\frac{y}{F(x, y)} \in \partial \Omega, \quad y \in T_{x} \Omega \approx \mathbb{R}^{n} .
$$

Em particular, se $\Omega=\mathbb{B}^{n}(1)$, temos

$$
F(x, y)=\frac{\sqrt{|y|^{2}-\left(|x|^{2}|y|^{2}-<x, y>^{2}\right)}+<x, y>}{1-|x|^{2}} .
$$


Antes de dar a conhecer alguns exemplos mais complexos, enunciaremos o seguinte Teorema que é de uso frequente nos argumentos de muitos resultados.

Teorema 1.1.1. (Teorema de Euler) Suponha que a função $H$ sobre $\mathbb{R}^{n}$ é diferenciável fora do origem. Então as seguintes afirmações são equivalentes:

- H é positiva homogênea de grau r, isto é,

$$
H(\lambda y)=\lambda^{r} H(y), \quad \text { para todo } \lambda>0
$$

- A derivada direcional radial de $H$ é $r$ vezes $H(r)$, isto é,

$$
y^{i} H_{y^{i}}(y)=r H(y)
$$

\subsection{Métrica $(\alpha, \beta)$-generalizada}

De agora em diante sempre que seja possível, não faremos distinção entre $(x, y) \in$ $T M$ e seu sistema de representação de coordenadas locais $\left(x^{i}, y^{i}\right)$, isto é

$$
x=\left(x^{i}\right) \in M, \quad y=y^{i} \frac{\partial}{\partial x^{i}} \in T_{x} M .
$$

Métricas $(\alpha, \beta)$ formam uma classe especial de métricas de Finsler, particularmente porque elas são mais tratáveis [1]. As métricas de Randers são exemplos mais simples das $(\alpha, \beta)$-métricas, pois basta considerar $\phi(s)=1-s$. Outro exemplo importante deste tipo de métricas foi dado por L. Berwald [7],

$$
F=\frac{\left(\sqrt{\left(1-|x|^{2}\right)|y|^{2}+<x, y>^{2}}+<x, y>\right)^{2}}{\left(1-|x|^{2}\right)^{2} \sqrt{\left(1-|x|^{2}\right)^{2}|y|^{2}+<x, y>^{2}}} .
$$

que é um tipo especial das métricas $(\alpha, \beta)$ na forma $F=\frac{(\alpha+\beta)^{2}}{\alpha}$, com $\|\beta\|_{\alpha}<1$. Esta métrica é projetivamente plana sobre $\mathbb{B}^{n}(1)$ com curvatura flag $K=0$. O conceito de métricas $(\alpha, \beta)$ foi proposta pela primeira vez por M. Matsumoto em 1972 como uma direta generalização de métricas de Randers [19].

Em 2011, C.Yu e H. Zhu, [40] generalizaram o conceito da $(\alpha, \beta)$-métrica como segue.

Definição 1.2.1. [40] Seja $F$ uma métrica de Finsler sobre uma variedade $M . F$ é chamada $(\alpha, \beta)$-métrica generalizada, se $F$ pode ser expressa como $F=\alpha \phi\left(x, \frac{\beta}{\alpha}\right)$, onde $\phi(x, s)$ é uma função regular, $\alpha$ é uma métrica Riemanniana e $\beta$ é uma 1-forma. $F$ é chamada uma $(\alpha, \beta)$-métrica, se $F$ pode ser expressa como $F=\alpha \phi\left(\frac{\beta}{\alpha}\right)$, onde $\phi(s)$ é uma função regular, $\alpha$ uma métrica Riemanniana e $\beta$ uma 1-forma. 
As métricas de Finsler do tipo

$$
F=\alpha \phi\left(b, \frac{\beta}{\alpha}\right)
$$

são as classes mais tratáveis das $(\alpha, \beta)$ - métricas generalizadas a menos das $(\alpha, \beta)$-métricas. $\phi(b, s)$ é uma função positiva regular com $b, s$ como suas variáveis e $|s| \leq b<b_{0}$ como definição do seu domínio para $0<b_{0} \leq+\infty$.

Vamos citar resultados básicos para métricas do tipo (1.4) que foram obtidos em [40].

Proposição 1.2.1. [40] Seja $F=\alpha \phi\left(b, \frac{\beta}{\alpha}\right)$ uma $(\alpha, \beta)$-métrica generalizada, então o tensor fundamental é dado por

$$
g_{i j}=\rho a_{i j}+\rho_{0} b_{i} b_{j}+\rho_{1}\left(b_{i} \alpha_{y^{j}}+b_{j} \alpha_{y^{i}}\right)-s \rho_{1} \alpha_{y^{i}} \alpha_{y^{j}},
$$

onde $s=\frac{\beta}{\alpha} e$

$$
\rho=\phi\left(\phi-s \phi_{s}\right), \quad \rho_{0}=\phi \phi_{s s}+\phi_{s} \phi_{s}, \quad \rho_{1}=\left(\phi-s \phi_{s}\right) \phi_{s}-s \phi \phi_{s s} .
$$

Além disso,

$$
\begin{aligned}
\operatorname{det}\left(g_{i j}\right) & =\phi^{n+1}\left(\phi-s \phi_{s}\right)^{n-2}\left(\phi-s \phi_{s}+\left(b^{2}-s^{2}\right) \phi_{s s}\right) \operatorname{det}\left(a_{i j}\right), \\
g^{i j} & =\rho^{-1}\left\{a^{i j}+\eta b^{i} b^{j} \eta_{0} \alpha^{-1}\left(b^{i} y^{j}+b^{j} y^{i}\right)+\eta_{1} \alpha^{-2} y^{i} y^{j}\right\},
\end{aligned}
$$

onde $\left(g^{i j}\right)=\left(g_{i j}\right)^{-1},\left(a^{i j}\right)=\left(a_{i j}\right)^{-1}, b^{i}=a^{i j} b_{j}$,

$$
\begin{aligned}
& \eta=-\frac{\phi_{s s}}{\left(\phi-s \phi_{s}+\left(b^{2}-s^{2}\right) \phi_{s s}\right)}, \quad \quad \eta_{0}=-\frac{\left(\phi-s \phi_{s}\right) \phi_{s}-s \phi \phi_{s s}}{\phi\left(\phi-s \phi_{s}+\left(b^{2}-s^{2}\right) \phi_{s s}\right)}, \\
& \eta_{1}=\frac{\left(s \phi+\left(b^{2}-s^{2}\right) \phi_{s}\right)\left(\left(\phi-s \phi_{s}\right) \phi_{s}-s \phi \phi_{s s}\right)}{\phi^{2}\left(\phi-s \phi_{s}+\left(b^{2}-s^{2}\right) \phi_{s s}\right)} .
\end{aligned}
$$

Proposição 1.2.2. [40] Sejam $M$ uma variedade de dimensão n, a uma métrica Riemanniana e $\beta$ uma 1-forma com $\|\beta\|_{\alpha}<b_{0}$. Então $F=\alpha \phi\left(b, \frac{\beta}{\alpha}\right)$ é uma métrica de Finsler sobre $M$ se, e somente se, $\phi=\phi(b, s)$ é uma função diferenciável positiva satisfazendo

$$
\phi(s)-s \phi_{s}(s)+\left(r^{2}-s^{2}\right) \phi_{s s}(s)>0,
$$

quando $n \geq 2$, com a condição adicional

$$
\phi(s)-s \phi_{s}(s)>0
$$

quando $n \geq 3$. 


\subsection{Geodésicas e Campos de Vetores Paralelos}

\section{Geodésicas}

Nesta seção, daremos a equação tipo Euler-Lagrange para curvas minimizantes num espaço de Finsler $(M, F)$. Seja $c:[a, b] \rightarrow M$ uma curva $C^{\infty}$ por partes, com velocidade $F(\dot{c})=\lambda$, onde $\lambda$ é uma constante real. Por definição, existe uma partição de $[a, b]$,

$$
a=t_{0}<\ldots<t_{m}=b
$$

tal que $c(r)$ é $C^{\infty}$ sobre cada $\left[t_{i-1}, t_{i}\right]$. Fixemos a partição acima e consideremos uma variação $C^{\infty}$ por partes de $c(t)$ dada pela aplicação $H:(-\epsilon, \epsilon) \times[a, b] \rightarrow M$ tal que

a. $H$ é $C^{0}$ sobre $(-\epsilon, \epsilon) \times[a, b]$;

b. $H$ é $C^{\infty}$ sobre cada região $(-\epsilon, \epsilon) \times\left[t_{i-1}, t_{i}\right], i=1, \ldots, m$;

c. $c(t)=H(0, t), a \leq t \leq b$,

O campo vetorial

$$
V(t)=\left.V^{i}(t) \frac{\partial}{\partial x^{i}}\right|_{c(t)}:=\frac{\partial H}{\partial u}(0, t)
$$

é chamado campo variacional de $H$.

O comprimento de $c_{u}(t):=H(u, t)$ é dado por

$$
\mathcal{L}(u):=\int_{a}^{b} F\left(\dot{c}_{u}(t)\right) d t=\sum_{i=1}^{m} \int_{t_{i-1}}^{t_{i}} F\left(\frac{\partial H}{\partial t}(u, t)\right) d t
$$

Observe que

$$
\begin{aligned}
\mathcal{L}^{\prime}(0)= & \int_{a}^{b} \frac{1}{2 F}\left\{\left[F^{2}\right]_{x^{k}} V^{k}+\left[F^{2}\right]_{y^{k}} \frac{d V^{k}}{d t}\right\} d t \\
= & \int_{a}^{b}\left\{\frac{1}{2 F}\left[F^{2}\right]_{x^{k}}-\frac{d}{d t}\left(\frac{1}{2 F}\left[F^{2}\right]_{y^{k}}\right)\right\} V^{k} d t \\
& +\left.\sum_{i=1}^{m} \frac{1}{2 F}\left[F^{2}\right]_{y^{k}} V^{k}\right|_{t_{i-1}} ^{t_{i}} \\
= & \int_{a}^{b} \frac{1}{2 F}\left\{\left[F^{2}\right]_{x^{k}}-\left[F^{2}\right]_{x^{l} y^{k}} \dot{c}^{l}-\left[F^{2}\right]_{y^{k} y^{\prime}} \ddot{c}^{l}\right\} V^{k} d t \\
& +\left.\sum_{i=1}^{m} \frac{1}{2 F}\left[F^{2}\right]_{y^{k}} V^{k}\right|_{t_{i-1}} ^{t_{i}} \\
= & -\int_{a}^{b} \frac{1}{F} g_{j k}\left\{\ddot{c}^{j}+2 G^{j}(\dot{c})\right\} V^{k} d t \\
& +\left.\sum_{i=1}^{m} \frac{1}{2 F}\left[F^{2}\right]_{y^{k}} V^{k}\right|_{t_{i-1}} ^{t_{i}},
\end{aligned}
$$


onde $g_{i j}(y):=\frac{1}{2}\left[F^{2}\right]_{y^{i} y^{j}}(y) \mathrm{e}$

$$
G^{i}(y):=\frac{1}{4} g^{i l}\left\{\left[F^{2}\right]_{x^{k} y^{l}}(y) y^{k}-\left[F^{2}\right]_{x^{l}}(y)\right\} .
$$

Seja

$$
\kappa(t):=\left.\frac{1}{F(\dot{c}(t))^{2}}\left\{\ddot{c}^{i}+2 G^{i}(\dot{c})\right\} \frac{\partial}{\partial x^{i}}\right|_{c(t)} .
$$

$\kappa(t)$ é chamado curvatura geodésica de $c$ em $t$. Podemos expressar (1.5) sem os índices da seguinte forma

$$
\begin{aligned}
\mathcal{L}^{\prime}(0)= & -\lambda \int_{a}^{b} \mathbf{g}_{\dot{c}}(\kappa, V) d t+\lambda^{-1} \mathbf{g}_{\dot{c}(b)}(\dot{c}(b), V(b))-\mathbf{g}_{\dot{c}(a)}(\dot{c}(a), V(a)) \\
& +\lambda^{-1} \sum_{i=1}^{k-1}\left\{\mathbf{g}_{\dot{c}\left(t_{i}^{-}\right)}\left(\dot{c}\left(t_{i}^{-}\right), V\left(t_{i}\right)\right)-\mathbf{g}_{\dot{c}\left(t_{i}^{+}\right)}\left(\dot{c}\left(t_{i}^{+}\right), V\left(t_{i}\right)\right)\right\},
\end{aligned}
$$

onde $\lambda=F(\dot{c}(t))$ é constante por hipótese.

Assumamos que $c$ tem comprimento mínimo. Então $L^{\prime}(0)=0$, para quaisquer variação $C^{\infty}$ por partes $H$ de $c$ fixados os pontos inicial e final de $c$.

Primeiramente, consideremos qualquer variação $C^{\infty}$ por partes $H$ de $c$ com $H\left(u, t_{i}\right)=c\left(t_{i}\right)$ (portanto, $\left.V\left(t_{i}\right)=0\right), i=0, \ldots, k$. Por $(1.8)$, temos

$$
\mathcal{L}^{\prime}(0)=\lambda \int_{a}^{b} \mathbf{g}_{\dot{c}}(\kappa, V) d t=0
$$

Isto implica que

$$
\kappa(t)=0
$$

Para quaisquer $1 \leq i_{0} \leq k-1$ e $v \in T_{\dot{c}\left(t_{i_{0}}\right)}$, consideremos uma variação $C^{\infty}$ por partes $H$ de $c$, tal que deixa fixo os pontos inicial e final de $c$ com

$$
V\left(t_{i_{0}}\right)=v, \quad H\left(u, t_{i}\right)=c\left(t_{i}\right), \quad i \neq i_{0}
$$

Por (1.8), temos

$$
\mathcal{L}^{\prime}(0)=\lambda^{-1}\left\{\mathbf{g}_{\dot{c}\left(t_{i_{0}}^{+}\right)}\left(\dot{c}\left(t_{i_{0}}^{+}\right), v\right)-\mathbf{g}_{\dot{c}\left(t_{i_{0}}^{-}\right)}\left(\dot{c}\left(t_{i_{0}}^{-}\right), v\right)\right\}=0 .
$$

Concluímos que

$$
\dot{c}\left(t_{i_{0}}^{-}\right)=\dot{c}\left(t_{i_{0}}^{+}\right) \text {, }
$$

isto é, $c$ é $C^{1}$ em cada $t_{i}$. Em coordenadas locais, $\kappa=0$ é equivalente ao seguinte sistema de equações diferenciais ordinárias,

$$
\ddot{c}^{i}+2 G^{i}(\dot{c})=0, \quad i=1,2, \ldots n
$$

Por isso, $c$ deve ser $C^{\infty}$ em cada $t_{i}$. Com isso, temos o seguinte resultado. 
Proposição 1.3.1. Seja c uma curva $C^{\infty}$ por partes com velocidade constante sobre uma variedade de Finsler $(M, F)$. Se c é de comprimento mínimo, então c é uma curva $C^{\infty}$ com curvatura geodésica nula $(\kappa=0)$.

A Proposição 1.3.1 motiva a seguinte definição,

Definição 1.3.1. Uma curva $C^{\infty}$ numa variedade de Finsler $(M, F)$ é chamada geodésica se ela tem velocidade constante e sua curvatura geodésica é nula.

As funções locais $G^{i}$, chamadas coeficientes geodésicos, definidas em (1.6) podem ser expressas como (ver [13])

$$
G^{i}(y)=\frac{1}{4} g^{i l}(y)\left\{2 \frac{\partial g_{j l}}{\partial x^{k}}(y)-\frac{\partial g_{j k}}{\partial x^{l}}(y)\right\} y^{j} y^{k} .
$$

Usando

$$
\left[F^{2}\right]_{x^{l}}=2 F F_{x^{l}}, \quad\left[F^{2}\right]_{x^{k} y^{l}} y^{k}=\frac{2 F_{x^{k}} y^{k}}{F} g_{m l} y^{m}+2 F F_{x^{k} y^{l}} y^{k},
$$

obtemos

$$
G^{i}=P y^{i}+Q^{i}
$$

onde

$$
P:=\frac{F_{x^{k}} y^{k}}{2 F}, \quad Q^{i}:=\frac{F}{2} g^{i l}\left\{F_{x^{k} y^{l}} y^{k}-F_{x^{l}}\right\} .
$$

Da definição, os coeficientes geodésicos $G^{i}$ satisfazem a seguinte condição de homogeneidade

$$
G^{i}(\lambda y)=\lambda^{2} G^{i}(y), \quad \lambda>0 .
$$

\section{Campo de Vetores Paralelo}

Sejam $(M, F)$ uma variedade de Finsler, $c=c(t)$ uma curva $C^{\infty}$ em $M$ e $U=$ $\left.U^{i}(t) \frac{\partial}{\partial x^{i}}\right|_{c(t)}$ um campo vetorial ao longo de $c$. Defina

$$
D_{\dot{c}} U(t):=\left.\left\{\dot{U}^{i}(t)+U^{j}(t) N_{j}^{i}(c(t), \dot{c}(t))\right\} \frac{\partial}{\partial x^{i}}\right|_{c(t)},
$$

onde $N_{j}^{i}:=\frac{\partial G^{i}}{\partial y^{j}}$. Podemos verificar que

$$
\begin{aligned}
D_{\dot{c}}(U+V)(t) & =D_{\dot{c}} U(t)+D_{\dot{c}} V(t), \\
D_{\dot{c}}(f U)(t) & =f^{\prime}(t) U(t)+f(t) D_{\dot{c}} U(t) .
\end{aligned}
$$

Como $D_{\dot{c}} U(t)$ depende linearmente de $U=U(t), D_{\dot{c}} U(t)$ é chamado derivada covariante de $U(t)$ ao longo de $c(t)$. 
Um campo vetorial $U=U(t)$ ao longo de $c(t)$ é chamado de campo vetorial linearmente paralelo se satisfaz a equação $D_{\dot{c}} U(t)=0$, isto é,

$$
\dot{U}^{i}(t)+U^{j}(t) N_{j}^{i}(c(t), \dot{c}(t))=0
$$

É claro que, para qualquer $t_{0}$ no domínio, $U$ depende linearmente do valor inicial $U\left(t_{0}\right)$.

Seja $\sigma=\sigma(t)$ uma curva em $M$. Então o campo vetorial tangente $U:=\dot{\sigma}(t)$ é um campo vetorial especial ao longo de $\sigma$. Sendo $G^{i}$ homogêneo positivo de grau 2 , a equação (1.13) torna-se

$$
\ddot{\sigma}^{i}(t)+2 G^{i}(\sigma(t), \dot{\sigma}(t))=0
$$

Por isso, a curva $\sigma$ é uma geodésica se, e somente se, o campo vetorial tangente $U=\dot{\sigma}(t)$ é linearmente paralelo ao longo da curva $\sigma$.

Observação 1.3.1. Para campos vetoriais linearmente paralelos $X=X(t)$ e $Y=Y(t)$ ao longo de uma geodésica $c(t)$, a expressão $g_{\dot{c}(t)}(X(t), Y(t))$ é constante, e para um campo vetorial paralelo $X=X(t)$ ao longo de uma curva $c, F(c(t), X(t))$ é constante. A demonstração deste resultado pode ser encontrada em [13] pg. 73-74.

Duas métricas $F$ e $\bar{F}$ sobre uma variedade $M$ são chamadas equivalentes afim se elas têm as mesmas geodésicas como curva parametrizada, isto é, se $\sigma=\sigma(t)$ é uma geodésica de $F$, então é também uma geodésica de $\bar{F}$ e vice-versa. Sejam $G^{i}=G^{i}(x, y)$ e $\bar{G}^{i}=\bar{G}^{i}(x, y)$, os coeficientes geodésicos de $F$ e $\bar{F}$, respectivamente, no mesmo sistema canônico de coordenadas locais $\left(x^{i}, y^{i}\right)$ em $T M$. Claramente, $F$ e $\bar{F}$ são equivalentes afim se, e somente se,

$$
G^{i}(x, y)=\bar{G}^{i}(x, y)
$$

Seja $F$ uma métrica de Minkowski sobre um espaço vetorial. Então $F$ é equivalente afim a uma métrica Euclidiana.

Um resultado interessante e útil é o seguinte,

Lema 1.3.1. Sejam $(M, \bar{F})$ uma variedade de Finsler e $F$ uma outra métrica de Finsler sobre $M$ tal que para qualquer campo vetorial paralelo $U=U(t)$ com respeito a $\bar{F}$ ao longo de qualquer curva $c=c(t)$ se tenha,

$$
F(c(t), U(t))=\text { constante }
$$

então $F$ é equivalente afim a $\bar{F}$.

Usando o conceito de campo de vetores paralelos ao longo de uma curva, podemos definir o transporte paralelo. 
Definição 1.3.2. Seja $c=c(t), a \leq t \leq b$, uma curva $C^{\infty}$ por partes de $c(0)=p$ a $c(b)=q$. Defina $P_{c}: T_{p} M \rightarrow T_{q} M$ por

$$
P_{c}(u):=U(b), \quad u \in T_{p} M,
$$

onde $U=U(t)$ é o campo vetorial paralelo ao longo de $c$ com $U(a)=u$. $P_{c}$ é chamado transporte paralelo ao longo de $c$.

O transporte paralelo $P_{c}$ é um difeomorfismo $C^{\infty}$ de $T_{p} M \backslash\{0\}$ sobre $T_{q} M \backslash\{0\}$ e é positivo homogêneo de grau 1 ,

$$
P_{c}(\lambda u)=\lambda P_{c}(u), \quad \lambda>0, u \in T_{p} M
$$

No entanto, em geral $P_{c}$ não é linear.

\subsection{Métricas de Berwald e Landsberg}

\section{Métricas de Berwald}

De forma geral, os coeficientes geodésicos $G^{i}$ não são quadráticos em $y \in T_{x} M$.

Definição 1.4.1. Uma métrica de Finsler é chamada métrica de Berwald se para qualquer sistema canônico de coordenadas locais $\left(x^{i}, y^{i}\right)$, os coeficientes geodésicos $G^{i}$ são quadráticos em $y \in T_{x} M$, para todo $x \in M$, ou seja, existem funções locais $\Gamma_{j k}^{i}$ sobre $M$ tal que

$$
G^{i}(y)=\frac{1}{2} \Gamma_{j k}^{i}(x) y^{j} y^{k}
$$

No exemplo seguinte, veremos que toda métrica Riemanniana é de Berwald:

Exemplo 1.4.1. Seja $F(x, y)=\sqrt{g_{i j} y^{i} y^{j}}$ uma métrica Riemanniana sobre uma variedade M. De (1.11) temos

$$
G^{i}(x, y)=\frac{1}{2} g^{i l}(x)\left\{2 \frac{\partial g_{j l}}{\partial x^{k}}(x)-\frac{\partial g_{j k}}{\partial x^{l}}(x)\right\} y^{j} y^{k},
$$

onde $\left(g^{i j}(x)\right):=\left(g_{i j}(x)\right)^{-1}$. Claramente, $G^{i}$ é quadrático em $y \in T_{x} M$. Logo $F$ é uma métrica de Berwald.

Existem muitas métricas de Berwald que não são Riemannianas.

Exemplo 1.4.2. Considere a métrica de Randers $F=\alpha+\beta$ sobre uma variedade $M$, onde $\alpha(x, y)=\sqrt{a_{i j}(x) y^{i} y^{j}}$ é Riemanniana e $\beta(x, y)=b_{i}(x) y^{i}$ é uma 1-forma com $\|\beta\|_{x}<1$. Como foi obtido no exemplo anterior, temos que os coeficientes geodésicos de $\alpha$ podem ser expressos como

$$
\bar{G}^{i}(x, y)=\frac{1}{2} \bar{\Gamma}_{i k}^{j} y^{j} y^{k}
$$


onde $\bar{\Gamma}_{j k}^{i}(x)=\bar{\Gamma}_{k j}^{i}(x)$ são funções locais de $x \in M$. Defina $b_{i \mid j}$ por

$$
b_{i \mid j} d x^{j}:=d b_{i}-b_{j} \bar{\Gamma}_{i k}^{j} d x^{k} .
$$

Sejam

$$
r_{i j}:=\frac{1}{2}\left(b_{i \mid j}+b_{j \mid i}\right), \quad s_{i j}:=\frac{1}{2}\left(b_{i \mid j}-b_{j \mid i}\right) .
$$

Os coeficientes geodésicos $G^{i}$ de $F$ são dados por

$$
G^{i}=\bar{G}^{i}+P y^{i}+Q^{i}
$$

onde

$$
\begin{gathered}
P:=\frac{1}{2 F}\left\{r_{i j} y^{i} y^{j}-2 \alpha b_{r} a^{r p} s_{p l} y^{l}\right\} \\
Q^{i}:=\alpha a^{i r} s_{r l} y^{l} .
\end{gathered}
$$

Assumamos que $\beta$ seja paralelo com respeito a $\alpha$, isto é, $b_{i \mid j}=0$, então

$$
r_{i j}=0=s_{i j}
$$

Portanto, $P=Q^{i}=0$, o que implica

$$
G^{i}=\bar{G}^{i}
$$

Como os $G^{i}$ são quadráticos em $y \in T_{x} M$ para todo $x \in M$, segue que $F=\alpha+\beta$ é uma métrica de Berwald. Neste caso as geodésicas de $F$ coincidem com as de $\alpha$ a menos de uma reparametrização. Por outro lado, se $F$ é do tipo Berwald, então $b_{i \mid j}=0$. Ver [20] para maiores detalhes.

É por isso que se uma métrica de Finsler é equivalente afim a uma métrica Riemanniana, então deve ser uma métrica de Berwald.

Proposição 1.4.1. [16] Seja $(M, F)$ uma variedade de Berwald. Para qualquer curva regular $c(t)$ de p a q em $M$, o transporte paralelo $P_{c}$ é uma isometria linear entre $\left(T_{p} M, F_{p}\right)$ $e\left(T_{q} M, F_{q}\right)$.

As métricas Riemannianas são uma família especial das métricas de Berwald. Na verdade, métricas de Berwald são quase Riemannianas, no sentido de que toda métrica de Berwald é equivalente afim a uma métrica Riemanniana, isto é, que as geodésicas de qualquer métrica de Berwald são geodésicas de alguma métrica Riemanniana [37].

Uma caracterização equivalente das métricas de Berwald e que foi assim como L. Berwald definiu pela primeira vez (ver [5] [6]), é a seguinte:

Seja

$$
B_{j k l}^{i}(y):=\frac{\partial G^{i}}{\partial y^{j} \partial y^{k} \partial y^{l}}(y)
$$


onde $G^{i}$ são os coeficientes geodésicos de $F$. Para um vetor tangente $y \in T_{x} M \backslash\{0\}$, defina

$$
B_{y}: T_{x} M \otimes T_{x} M \otimes T_{x} M \rightarrow T_{x} M
$$

por

$$
B_{y}(u, v, w)=\left.B_{j k l}^{i}(y) u^{j} v^{k} w^{l} \frac{\partial}{\partial x^{i}}\right|_{x}
$$

onde $u=\left.u^{i} \frac{\partial}{\partial x^{i}}\right|_{x}, v=\left.v^{j} \frac{\partial}{\partial x^{j}}\right|_{x}$ e $w=\left.w^{k} \frac{\partial}{\partial x^{k}}\right|_{x} . B_{y}(u, v, w)$ é simétrico em $u, v$ e $w$. A homogeneidade de $G^{i}$ implica

$$
B_{y}(y, v, w)=0
$$

Definição 1.4.2. [31] A curvatura de Berwald de uma métrica de Finsler é definida como um tensor, que em coordenadas locais é dado por:

$$
B:=B_{j k l}^{i} d x^{j} \otimes d x^{k} \otimes d x^{l} \otimes \frac{\partial}{\partial x^{i}},
$$

onde,

$$
B_{j k l}^{i}=\frac{\partial^{3} G^{i}}{\partial y^{j} \partial y^{k} \partial y^{l}}
$$

e $G^{i}$ são os coeficientes geodésicos de $F$.

Pode-se mostrar (ver [32]) que uma métrica de Finsler é de Berwald se, e somente se, sua curvatura de Berwald é nula, pois

$$
\Gamma_{j k}^{i}=\frac{\partial^{2} G^{i}}{\partial y^{j} \partial y^{k}} .
$$

De agora em diante diremos que uma métrica de Finsler é de Berwald se, e somente se, para todo índice $i, j, k, l$ e $(x, y) \in T M$

$$
\frac{\partial^{3} G^{i}}{\partial y^{j} \partial y^{k} \partial y^{l}}=0
$$

onde $G^{i}$ são os coeficientes geodésicos de $F$ dados por (1.6) ou (1.12)

\section{Métricas de Landsberg}

Seja $(M, F)$ uma variedade de Finsler. Para $y \in T_{x} M$, defina

$$
L_{y}(u, v, w):=\frac{1}{2} g_{y}\left(B_{y}(u, v, w), y\right) .
$$


Em coordenadas locais,

$$
L_{y}(u, v, w)=L_{i j k}(y) u^{i} v^{j} w^{k}
$$

onde $u=\left.u^{i} \frac{\partial}{\partial x^{i}}\right|_{x}, v=\left.v^{j} \frac{\partial}{\partial x^{j}}\right|_{x}, w=\left.w^{k} \frac{\partial}{\partial x^{k}}\right|_{x} \mathrm{e}$

$$
L_{i j k}(y)=\frac{1}{2} y^{m} g_{m l}(y) B_{i j k}^{l}(y)=\frac{1}{2} y^{m} g_{m l}(y) \frac{\partial^{3} G^{l}}{\partial y^{i} \partial y^{j} \partial y^{k}}(y) .
$$

$L_{y}$ é uma forma multilinear simétrica. Mostra-se facilmente de (1.20) e (1.22) que

$$
L_{y}(y, v, w)=0
$$

Definição 1.1. L é dita Curvatura de Landsberg. Uma métrica de Finsler é dita métrica de Landsberg se $L=0$.

L. Berwald foi o primeiro a chamar as métricas com $L=0$ de métricas de Landsberg [6]. De (1.22) temos que se $B=0$, então $L=0$.

Portanto, as métricas de Berwald são métricas de Landsberg.

Sendo $F$ homogênea positiva de grau 1 , temos que a expressão de $L_{j k l}$ pode ser melhorada como

$$
L_{j k l}(y)=\frac{1}{2} F F_{y^{i}} B_{j k l}^{i}
$$

Exemplo 1.4.3. Consideremos a métrica de Randers $F:=\alpha+\beta$, onde $\alpha(x, y)=$ $\sqrt{a_{i j}(x) y^{i} y^{j}}$ é uma métrica Riemanniana e $\beta(x, y)=b_{i}(x) y^{i}$ é uma 1 -forma sobre $M$. M. Matsumoto, em [20], provou que $F$ é uma métrica de Landsberg se, e somente se, $\beta$ é paralelo com respeito a $\alpha$. Isto é,

$$
\frac{\partial b_{i}}{\partial x^{j}}-\Gamma_{i j}^{k} b_{k}=0
$$

onde $\Gamma_{i j}^{k}$ são os símbolos de Christoffel da métrica $\alpha$.

Na geometria de Finsler existe um problema que ainda está em aberto sobre as métricas de Berwald e Landsberg: verificar se existe uma métrica de Landsberg que não é uma métrica de Berwald. Este problema foi chamado "problema do unicórnio" por D. Bao e M. Matsumoto declarou que procurar tal uma métrica representa o próximo objetivo da Geometria de Finsler [3]. Em 2008, Z. I. Szabó afirmou que toda métrica de Landsberg é do tipo Berwald [35]. Porém, existe um erro na demonstração, pode-se consultar [36] e [21] para mais detalhes.

É conhecido que as seguintes três condições são equivalentes para uma métrica de Randers $F=\alpha+\beta,(\operatorname{ver}[20])$

(a) F é métrica de Landsberg; 
(b) F é métrica de Berwald;

(c) $\beta$ é paralelo com respeito a $\alpha$.

Z. Shen mostrou que as métricas de Landsberg do tipo $(\alpha, \beta)$-métricas são também métricas de Berwald [33].

\subsection{Métricas de Douglas}

A noção de espaços de Douglas foi introduzida por S. Bacso e M. Matsumoto [2] como uma generalização dos espaços de Berwald do ponto de vista das equações geodésicas. Uma métrica de Finsler é chamada de métrica de Douglas se os coeficientes geodésicos $G^{i}=G^{i}(x, y)$ são da seguinte forma:

$$
G^{i}=\frac{1}{2} \Gamma_{j k}^{i}(x) y^{j} y^{k}+O(x, y) y^{i},
$$

onde $\Gamma_{j k}^{i}$ são funções de $x \in M$ e $P$ uma função de $(x, y) \in T M$ que satisfaz a seguinte propriedade de homogeneidade

$$
O(\lambda y)=\lambda O(y)
$$

As métricas de Douglas formam uma família muito rica, incluindo as métricas Riemannianas e as métricas localmente projetivamente planas (métricas que têm linhas retas como geodésicas). O estudo das métricas de Douglas enriquecem nosso entendimento das métricas de Finsler não Riemannianas.

Seja

$$
D_{j k l}^{i}:=\frac{\partial^{3}}{\partial y^{j} \partial y^{k} \partial y^{l}}\left(G^{i}-\frac{1}{n+1} \frac{\partial G^{m}}{\partial y^{m}} y^{i}\right) .
$$

Observe que a condição (1.27) é satisfeita se, e somente se,

$$
G^{i}-\frac{1}{n+1} \frac{\partial G^{m}}{\partial y^{m}} y^{i}=\gamma_{j k}^{i} y^{j} y^{k}
$$

para algum conjunto de funções $\gamma_{j k}^{i}(x)$. Além disso, (1.29) é satisfeita se, e somente se,

$$
D_{j k l}^{i}=0 .
$$

Por isso, as métricas de Douglas são também caracterizadas pela equação $D_{j}^{i}{ }_{k l}=0$. Por exemplo, uma métrica de Randers $F=\alpha+\beta$ é do tipo Douglas se, e somente se, $\beta$ é uma 1-forma fechada [2]. Neste caso $F=\alpha+\beta$ tem as mesmas geodésicas que $\alpha$.

De forma geral, considere-se a métrica de Finsler da forma

$$
F=\sqrt{\alpha^{2}+\kappa \beta^{2}}+\epsilon \beta,
$$


onde $\kappa$ e $\epsilon \neq 0$ são constantes. Claramente, $F=\sqrt{\alpha^{2}+\kappa \beta^{2}}+\epsilon \beta$ é do tipo Douglas se, e somente se, $\beta$ é fechado. Estes tipos de métricas motivam o seguinte Teorema, para métricas $(\alpha, \beta)$ sobre um aberto de dimensão $n \geq 3$.

Teorema 1.5.1. [17] Seja $F=\alpha \phi(s), s=\beta / \alpha$, uma $(\alpha, \beta)$ métrica sobre um aberto $\mathcal{U} \subset \mathbb{R}^{n}(n \geq 3)$, onde $\alpha=\sqrt{a_{i j}(x) y^{i} y^{j}}$ e $\beta=b_{i}(x) y^{i} \neq 0$. Defina $b:=\left\|\beta_{x}\right\|_{\alpha}$ e suponha as seguintes condições:

(a) $\beta$ não é paralelo com respeito a métrica $\alpha$,

(b) F não é do tipo Randers e

(c) $d b \neq 0$ em toda parte ou $b=$ constante sobre $\mathcal{U}$.

Então $F$ é do tipo Douglas sobre $\mathcal{U}$ se, e somente se, a função $\phi=\phi(s)$ satisfaz a seguinte equação diferencial ordinária:

$$
\left\{1+\left(\kappa_{1}+\kappa_{2} s^{2}\right) s^{2}+\kappa_{3} s^{2}\right\} \phi^{\prime \prime}(s)=\left(\kappa_{1}+\kappa_{2}\right)\left\{\phi(s)-s \phi^{\prime}(s)\right\}
$$

e a derivada covariante $\nabla \beta=b_{i \mid j} y^{i} d x^{j}$ de $\beta$ com respeito a $\alpha$ satisfaz a seguinte equação:

$$
b_{i \mid j}=2 \tau\left\{\left(1+\kappa_{1} b^{2}\right) a_{i j}+\left(\kappa_{2} b^{2}+\kappa_{3}\right) b_{i} b_{j}\right\}
$$

onde $\tau=\tau(x)$ é uma função escalar sobre $\mathcal{U}$ e $\kappa_{1}, \kappa_{2}$ e $\kappa_{3}$ são constantes com $\left(\kappa_{1}, \kappa_{2}\right) \neq$ $(0,0)$.

A equação (1.30) implica que $\beta$ é uma 1-forma fechada. Existem muitas soluções elementares de (1.30). Por exemplo, as seguintes funções $\phi$ satisfazem (1.30) para algumas constantes $\kappa_{i}$.

$$
\begin{gathered}
b \phi=1+s, \quad \phi=1+\epsilon s+s^{2}, \quad \phi=(1+s)^{2}, \\
\phi=1+\epsilon s+s \arctan (s), \quad \phi=1+\epsilon s+2 s^{2}-\frac{1}{3} s^{4},
\end{gathered}
$$

onde $\epsilon$ é constante.

As funções $\phi=e^{s}+\epsilon s$ e $\phi=1 /(1-s)+\epsilon s$ não satisfazem (1.30) para quaisquer $\kappa_{i}$. Consequentemente, as $(\alpha, \beta)$-métricas definidas por estas funções são métricas de Douglas se, e somente se, $\beta$ é paralela com respeito a $\alpha$. [4] [41]

Observação 1.5.1. O tensor de Douglas é projetivamente invariante, isto é, se duas métricas de Finsler $F$ e $\bar{F}$ são projetivamente equivalentes

$$
G^{i}=\bar{G}^{i}+P y^{i}
$$


onde $P=P(x, y)$ é positivo $y$-homogêneo de grau 1 , então o tensor de Douglas de $F$ é igual ao da $\bar{F}$. Por isso, se uma métrica de Finsler é projetivamente equivalente a uma métrica de Berwald, então ela é uma métrica de Douglas. Entretanto, existe o problema de saber se toda métrica de Douglas é (localmente) projetivamente equivalente a uma métrica de Berwald.

Exemplo 1.5.1. [18] A seguinte métrica de Finsler definida sobre a bola unitária $\mathbb{B}^{n} \subset \mathbb{R}^{n}$ é do tipo Douglas

$$
F(x, y)=\frac{\sqrt{\kappa^{2}<x, y>^{2}+\varepsilon|y|^{2}\left(1+\zeta|x|^{2}\right)}}{1+\zeta|x|^{2}}+\frac{\kappa<x, y>}{1+\zeta|x|^{2}},
$$

onde $r=1 / \sqrt{-\zeta}$ se $\zeta<0$ e $r=+\infty$ se $\zeta \geq 0$. $F$ não é Riemanniana (resp. localmente projetiva flat), a não ser que $\kappa=0$ (resp. $\left.\varepsilon \zeta+\kappa^{2}=0\right)$.

A seguinte métrica não é do tipo Randers em geral.

Exemplo 1.5.2. [28] A seguinte métrica de Finsler é do tipo Douglas e tem curvatura flag constante negativa

$$
\begin{aligned}
F_{\varepsilon}(x, y)= & \frac{1}{2}\left\{\frac{\sqrt{\left(1-|x|^{2}\right)|y|^{2}+<x, y>^{2}}+<x, y>}{1-|x|^{2}}\right\}- \\
& -\frac{1}{2}\left\{\frac{\varepsilon \sqrt{\left(1-\varepsilon|x|^{2}\right)|y|^{2}+\varepsilon^{2}<x, y>^{2}}+\varepsilon^{2}<x, y>}{1-\varepsilon^{2}|x|^{2}}\right\} .
\end{aligned}
$$

Note que $F_{\varepsilon}$ é do tipo Randers se $\varepsilon=0,-1$.

\section{$1.6 S-$ Curvatura}

Existem duas importantes formas de volume na geometria de Finsler. Uma delas é a chamada forma de volume Busemann-Hausdorff e a outra é chamada forma de volume Holmes-Thompson. A forma de volume Busemann-Hausdorff $d V_{B H}$ é dada por $d V_{B H}(x)=\sigma_{B H}(x) d x$, onde

$$
\sigma_{B H}(x)=\frac{\omega_{n}}{\operatorname{Vol}\left\{\left(y^{i}\right) \in R^{n} \mid F\left(x, y^{i} \frac{\partial}{\partial x^{i}}\right)<1\right\}}
$$

e a forma de volume Holmes-Thompson $d V_{H T}$ é dada por $d V_{H T}(x)=\sigma_{H T}(x) d x$, onde

$$
\sigma_{H T}(x)=\frac{1}{\omega_{n}} \int_{\left\{\left(y^{i}\right) \in R^{n} \mid F\left(x, y^{i} \frac{\partial}{\partial x^{i}}\right)<1\right\}} \operatorname{det}\left(g_{i j}\right) d y .
$$

O operador Vol denota o volume Euclidiano e

$$
\omega_{n}:=\operatorname{Vol}\left(B^{n}(1)\right)=\frac{1}{n} \operatorname{Vol}\left(S^{n-1}\right)=\frac{1}{n} \operatorname{Vol}\left(S^{n-2}\right) \int_{0}^{\pi} \sin ^{n-2}(t) d t
$$

denota o volume Euclidiano da bola unitária em $\mathbb{R}^{n}$. 
Proposição 1.6.1. Seja $F=\alpha \phi(\beta, \beta / \alpha)$ uma $(\alpha, \beta)$-métrica generalizada sobre uma variedade $M, n$-dimensional. Sejam $d V=d V_{B H}$ ou $d V_{H T} e$

$$
A(b):= \begin{cases}\frac{\int_{0}^{\pi} \sin ^{n-2}(t) d t}{\int_{0}^{\pi} \frac{\operatorname{sen}^{n-2}(t)}{\phi(b, b \cos (t))^{n}} d t}, & \text { se } d V=d V_{B H} \\ \frac{\int_{0}^{\pi}\left(\operatorname{sen}^{n-2}(t)\right) T(b \cos (t)) d t}{\int_{0}^{\pi} \operatorname{sen}^{n-2}(t) d t}, & \text { se } d V=d V_{H T},\end{cases}
$$

onde $T(s):=\phi\left(\phi-s \phi_{2}\right)^{n-2}\left[\left(\phi-s \phi_{2}\right)+\left(b^{2}-s^{2}\right) \phi_{22}\right]$. Então a forma de volume $d V$ é dada por

$$
d V=A(b) d V_{\alpha}
$$

onde $d V_{\alpha}=\sqrt{\operatorname{det}\left(a_{i j}\right)} d x$ denota a forma de volume Riemanniana de $\alpha$.

Considere o espaço euclidiano $\mathbb{R}^{n}$, e a família de formas de volume $d \mu_{\epsilon}=\sigma_{\epsilon}(x) d x^{1} \ldots d x^{n}$ sobre $\mathbb{R}^{n}$, onde

$$
\sigma_{\epsilon}=\epsilon^{n} e^{\epsilon|x|}, \quad \epsilon>0 .
$$

$d \mu_{\epsilon}$ determina as conhecidas medidas de Gauss $\mu_{\epsilon}$. Note que $\sigma_{\epsilon} \rightarrow 0$ quando $\epsilon \rightarrow 0^{+}$. Para um valor de $\epsilon$ fixado, a taxa de decaimento da medida Gaussiana $\mu_{\epsilon}$ em $x$ é definida por

$$
S_{x}(y):=-\frac{y^{i}}{\sigma_{\epsilon}(x)} \frac{\partial \sigma_{\epsilon}}{\partial x^{i}}(x)=2 \epsilon<x, y>, \quad y \in T_{x} \mathbb{R}^{n}=\mathbb{R}^{n} .
$$

A taxa de decaimento $S_{x}$ de $\mu_{\epsilon}$ vai para $\infty$ (na direção radial) quando $x \rightarrow \infty$. Podemos estender a noção de taxa de decaimento para variedades de Finsler.

Seja $(F, M)$ uma variedade de Minkowski. Considere um sistema de coordenadas locais $\left(x^{i}, y^{i}\right)$ em $T M$, e sejam $G^{i}$ os coeficientes geodésicos de $F$.

Se $F$ é uma norma de Minkowski, então $g_{i j}:=\frac{1}{2}\left[F^{2}\right]_{y^{i} y^{j}}(y)$ depende de $y$ e $\sigma_{F} \neq \sqrt{\operatorname{det}\left(g_{i j}(y)\right)}$, em geral. Defina

$$
\tau:=\ln \frac{\sqrt{\operatorname{det}\left(g_{i j}(y)\right)}}{\sigma_{F}} .
$$

$\tau=\tau(y)$ está bem definido, e é chamado de distorção de $F$ [30], [32]. Observe que

$$
\tau_{y^{i}}=\frac{\partial}{\partial y^{i}}\left[\frac{\ln \sqrt{\operatorname{det}\left(g_{j k}(y)\right)}}{\sigma_{F}}\right]=\frac{1}{2 \sigma_{F}} g^{j k} \frac{\partial g_{j k}}{\partial y^{i}} .
$$

Uma métrica Euclidiana é caracterizada pelo seguinte lema.

Lema 1.6.1. [13] Para uma norma de Minkowski $F$ sobre um espaço vetorial $V$, as seguintes condições são equivalentes: 
(a) Fé Euclidiana,

(b) $\tau=$ constante,

(c) $\tau=0$.

Seja $(M, F)$ uma variedade de Finsler. A distorção é definida para a norma de Minkowski sobre cada espaço tangencial. Então obtemos uma função escalar $\tau=\tau(x, y)$ sobre $T M \backslash\{0\}$, que também é chamada distorção de $F$. Então $F$ é Riemanniana se, e somente se, $\tau=0$.

Mostraremos que sobre uma variedade de Berwald, a distorção é constante ao longo de qualquer geodésica, porém não é constante sobre a variedade em geral. Por isso é natural estudar a variação da distorção ao longo de geodésicas para variedades de Finsler em geral.

Para um vetor $y \in T_{x} M \backslash\{0\}$, seja $c=c(t)$ uma geodésica com $c(0)=x$ e $\dot{c}(0)=y$. Defina

$$
S(x, y):=\left.\frac{d}{d t}[\tau(c(t), \dot{c}(t))]\right|_{t=0} .
$$

$S=S(x, y)$ é positiva $y$-homogênea de grau 1 ,

$$
S(x, \lambda y)=\lambda S(x, y), \quad \lambda>0 .
$$

$S$ é chamado de $S$-curvatura. Num sistema de coordenadas locais $\left(x^{i}, y^{i}\right)$, seja $d V_{F}=$ $\sigma_{F}(x) d x^{1} \ldots d x^{n}$ a forma de volume e $G^{i}=G^{i}(x, y)$ os coeficientes geodésicos de $F$. Temos de (1.15) que

$$
\frac{\partial G^{m}}{\partial y^{m}}=\frac{1}{2} g^{m l} \frac{\partial g_{m l}}{\partial x^{i}} y^{i}-g^{j k} \frac{\partial g_{j k}}{\partial y^{i}} G^{i}
$$

Portanto, de (1.35) e (1.36), temos

$$
\begin{aligned}
S & =y^{i} \frac{\partial \tau}{\partial x^{i}}-2 \frac{\partial \tau}{\partial y^{i}} G^{i} \\
& =\frac{1}{2} g^{m l} \frac{\partial g_{m l}}{\partial x^{i}} y^{i}-g^{j k} \frac{\partial g_{j k}}{\partial y^{i}} G^{i}-y^{m} \frac{\partial}{\partial x^{m}}\left(\ln \sigma_{F}(x)\right) \\
& =\frac{\partial G^{m}}{\partial y^{m}}(x, y)-y^{m} \frac{\partial}{\partial x^{m}}\left(\ln \sigma_{F}(x)\right) .
\end{aligned}
$$

Proposição 1.4.1 nos diz que toda variedade de Berwald é modelada sobre um único espaço de Minkowski. Além disso, a geometria dos espaços tangentes não muda ao longo das geodésicas. Esta observação leva a seguinte proposição importante para uma métrica de Berwald com respeito a forma de volume Busemann-Hausdorff $d V_{B H}$.

Proposição 1.6.2. [30] [31] Para qualquer métrica de Berwald, a S-curvatura é nula. 
Demonstração. Fixemos um ponto arbitrário $(x, y) \in T M_{0}$ e seja $c=c(t)$ uma geodésica com $c(0)=x$ e $\dot{c}(0)=y$. Seja $\left\{\mathbf{b}_{i}(t)\right\}$ um referencial linearmente paralelo ao longo de $c$, isto é, cada $\mathbf{b}_{i}(t)$ é linearmente paralelo ao longo de $c$. Seja

$$
g_{i j}(t):=\mathbf{g}_{\dot{c}(t)}\left(\mathbf{b}_{i}(t), \mathbf{b}_{j}(t)\right) \text {. }
$$

Pela Observação 1.3.1, $g_{i j}(t)=$ constante. Por isso $\operatorname{det}\left(g_{i j}(t)\right)=$ constante. Por outro lado, para quaisquer $\left(y^{i}\right) \in \mathbb{R}^{n}$, o campo vetorial $U=y^{i} \mathbf{b}_{i}(t)$ é linearmente paralelo ao longo de $\sigma$ e do Lema 1.3.1,

$$
F\left(c(t), y^{i} \mathbf{b}_{i}(t)\right)=\text { constante }
$$

Assim o subconjunto convexo $\mathcal{U}_{t} \subset \mathbb{R}^{n}$ é independente de $t$,

$$
\mathcal{U}_{t}:=\left\{\left(y^{i}\right) \in \mathbb{R}^{n} \mid F\left(c(t), y^{i} \mathbf{b}_{i}(t)\right)<1\right\} .
$$

Isto implica que o coeficiente da forma de volume $d V_{F}$ é constante,

$$
\sigma_{F}(t)=\frac{\operatorname{Vol}\left(\mathbb{B}^{n}(1)\right)}{\operatorname{Vol}\left(\mathcal{U}_{t}\right)}=\text { constante }
$$

Assim, a distorção também é constante, isto é,

$$
\tau(c(t), \dot{c}(t))=\ln \frac{\sqrt{\operatorname{det}\left(g_{i j}(t)\right)}}{\sigma_{F}(\sigma(t))}=\text { constante. }
$$

Logo, por (1.33) e (1.35), $S=0$.

A função $S$ foi originalmente definida por Z. Shen ([30] [29]) para variedades de Finsler com a medida de Busemann-Hausdorff.

Uma métrica de Finsler $F$ é chamada de $S$-curvatura isotrópica se

$$
S=(n+1) c F
$$

De forma mais geral, $F$ tem $S$-curvatura quase isotrópica se

$$
S=(n+1)(c F+\eta)
$$

onde $c=c(x)$ é uma função de $x \in M$ e $\eta=\eta_{i}(x) y^{i}$ é uma 1-forma fechada.

Observamos que, se os coeficientes geodésicos $G^{i}(y)$ são quadráticos em $y \in T_{x} M$, $x \in M$, então $S$ torna-se uma 1-forma sobre $M$.

Pela definição de $S$-curvatura, $S(y)$ mede a taxa de variação de $\left(T_{x} M, F_{x}\right)$ na direção $y \in T_{x} M$. 
Exemplo 1.6.1. [32] Sejam $\Omega$ um domínio convexo em $\mathbb{R}^{n}$ e a métrica de Funk definida por

$$
x+\frac{y}{F(x, y)} \in \partial \Omega, \quad y \in T_{x} \Omega .
$$

Considerando a base ortonormal canônico $\left\{e_{i}\right\}_{i=1}^{n}$ para $\mathbb{R}^{n}$, segue da definição que

$$
B_{x}^{n}:=\left\{\left(y^{i}\right) \in \mathbb{R}^{n}, F\left(x, y^{i} e_{i}\right) \leq 1\right\}=\bar{\Omega}-\{x\} .
$$

Segue que o elemento de volume $\sigma_{B H}(x)$ é dado por

$$
\sigma(x)=\frac{\operatorname{Vol}\left(\mathbb{B}^{n}\right)}{\operatorname{Vol}\left(B_{x}^{n}\right)}=\frac{\operatorname{Vol}\left(\mathbb{B}^{n}\right)}{\operatorname{Vol}(\Omega)}=\text { constante }
$$

Por outro lado o Teorema de Okada [25] para métricas de Funk $\left(F_{x^{k}}=F F_{y^{k}}\right)$ nos dá os coeficientes geodésicos $G^{i}$ de $F$ que são dados por

$$
G^{i}=\frac{1}{2} F y^{i} .
$$

Derivando (1.39), temos

$$
\frac{\partial G^{m}}{\partial y^{m}}=\frac{n+1}{2} F
$$

Assim, segue de (1.37) que

$$
S=\frac{n+1}{2} F
$$

Neste caso a $S$-curvatura é isotrópica constante, já que a função $c(x)=\frac{1}{2}$ em (1.38).

Exemplo 1.6.2. Considere a métrica de Randers $F=\alpha+\beta$ sobre uma variedade $M$, onde $\alpha(y)=\sqrt{a_{i j} y^{i} y^{j}}$ é uma métrica Riemanniana e $\beta(y)=b_{i} y^{i}$ é uma 1-forma com

$$
\|\beta\|_{\alpha}(x):=\sup _{y \in T_{x} M} \frac{\beta(y)}{\alpha(y)}=\sqrt{a^{i j} b_{i} b_{j}}<1 .
$$

Em [10] X. Chen e Z. Shen mostraram que $F$ tem $S$-curvatura isotrópica, isto é, $S=$ $(n+1) c F$, se, e somente se, $\beta$ satisfaz a seguinte equação:

$$
r_{i j}+b_{i} v_{j}+b_{j} v_{i}=2 c\left(a_{i j}-b_{i} b_{j}\right)
$$

onde

$$
r_{i j}:=\frac{1}{2}\left(b_{i \mid j}+b_{j \mid i}\right), \quad v_{i j}:=\frac{1}{2}\left(b_{i \mid j}-b_{j \mid i}\right), \quad v_{i}:=b^{j} v_{j i} .
$$

Aqui $b_{i \mid j}$ denotam os coeficientes da derivada covariante de $\beta$ com respeito a $\alpha$. Porém, obter soluções desta equação é difícil. Esta dificuldade será superada se expressarmos a métrica de Randers $F$ da seguinte forma

$$
F=\frac{\sqrt{h(x, y)^{2}-\left[h\left(x, W_{x}\right)^{2} h(x, y)^{2}-<y, W_{x}>_{x}^{2}\right]}}{1-h\left(x, W_{x}\right)^{2}}-\frac{<y, W_{x}>_{h}}{1-h\left(x, W_{x}\right)^{2}},
$$


onde $h(x, y)=\sqrt{h_{i j}(x) y^{i} y^{j}}$ é uma métrica Riemanniana, $W$ é um campo vetorial sobre $M$ com $h\left(x, W_{x}\right)<1$ para todo $x \in M \mathrm{e}<,>_{h}$ denota o produto interno definido por $h$. Em [39], H. Xin, mostrou que $F$ tem $S$-curvatura isotrópica $S=(n+1) c F$ se, e somente se, $W$ satisfaz

$$
W_{i ; j}+W_{j ; i}=-4 c h_{i j}
$$

onde $W_{i}:=h_{i j} W^{j}$ e $W_{i ; j}$ denotam os coeficientes da derivada covariante de $W$ com respeito a $h$. Neste mesmo espírito (isto é, com os dados de navegação de Zermelo $h$ e $W_{x}$ ), em [12] X. Cheng e Z. Shen determinaram completamente a estrutura local de uma métrica de Randers com $S$-curvatura isotrópica e com curvatura flag escalar $K=K(x, y)$.

Consideremos as seguintes notações

$$
v_{0}=v_{j} y^{j}, \quad v_{00}=v_{k l} y^{k} y^{l}, \quad v_{j}^{i}=a^{i h} v_{h j}, \quad v_{0}^{i}=v_{j}^{i} y^{j}, \quad r_{00}=r_{i j} y^{i} y^{j} .
$$

onde $r_{i j}, v_{i j}$ e $v_{i}$ são dados em (1.40).

Exemplo 1.6.3. Consideremos $s=\frac{\beta}{\alpha}$ e a métrica $F=\alpha \phi(s)$ chamada $(\alpha, \beta)$-métrica, os coeficientes geodésicos $G^{i}$ de $F$ são dados por

$$
G^{i}=\bar{G}^{i}+\alpha Q v_{0}^{i}+\Theta\left\{-2 Q \alpha v_{0}+r_{00}\right\} \frac{y^{i}}{\alpha}+\Psi\left\{-2 Q \alpha v_{0}+r_{00}\right\} b^{i},
$$

onde $\bar{G}^{i}$ denota os coeficientes geodésicos de $\alpha$ e

$$
Q:=\frac{\phi^{\prime}}{\phi-s \phi^{\prime}}, \quad \Theta=\frac{Q-s Q^{\prime}}{2 \Delta}, \quad \Psi=\frac{Q^{\prime}}{2 \Delta}
$$

onde $\Delta:=1+s Q+\left(b^{2}-s^{2}\right) Q^{\prime}$. Seja

$$
\Phi:=-\left(Q-s Q^{\prime}\right)\{n \Delta+1+s Q\}-\left(b^{2}-s^{2}\right)(1+s Q) Q^{\prime \prime}
$$

Em [11] X. Cheng e Z. Shen obtiveram equações que caracterizam as $(\alpha, \beta)$-métricas com $S$-curvatura isotrópica: Supondo $\phi \neq k_{1} \sqrt{1+k_{2} s^{2}}+k_{3} s$ para quaisquer constantes $k_{1}>0, k_{2}$ e $k_{3}$. Então $F$ tem $S$-curvatura isotrópica se, e somente se, ocorre um dos seguintes itens,

(i) $\beta$ satisfaz

$$
r_{j}+v_{j}=0
$$

e $\phi=\phi(s)$ satisfaz

$$
\Psi=0 \text {. }
$$

Neste caso, $S=0$. 
(ii) $\beta$ satisfaz

$$
r_{i j}=\epsilon\left\{b^{2} a_{i j}-b_{i} b_{j}\right\}, \quad v_{j}=0,
$$

onde $\epsilon=\epsilon(x)$ é uma função escalar, e $\phi=\phi(s)$ satisfaz

$$
\Psi=-2(n+1) k \frac{\psi \Delta^{2}}{b^{2}-s^{2}},
$$

onde $k$ é uma constante. Neste caso, $S=(n+1) c F \operatorname{com} c=k \epsilon$.

(iii) $\beta$ satisfaz

$$
r_{i j}=0, \quad v_{j}=0 .
$$

Neste caso, $S=0$, independente da escolha de um $\phi$ particular.

Por outro lado, para o caso $F=k_{1} \sqrt{\alpha^{2}+k_{2} \beta^{2}}+k_{3} \beta$, temos que $F$ tem $S$-curvatura isotrópica, $S=(n+1) c F$ se, e somente se, $\beta$ satisfaz

$$
r_{i j}+\tau\left(v_{i} b_{j}+v_{j} b_{i}\right)=\frac{2 c\left(1+k_{2} b^{2}\right) k_{1}^{2}}{k_{3}}\left(a_{i j}-\tau b_{i} b_{j}\right)
$$

onde

$$
\tau:=\frac{k_{3}^{2}}{k_{1}^{2}}-k_{2}
$$

Neste último exemplo foram consideradas as formas de volume Busemann- Hausdorff $d V_{B H}$ e de Holmes-Thompson $d V_{H T}$ dadas pela Proposição 1.6.1.

Outra propriedade interessante da S-curvatura é que ela está estreitamente relacionada com a curvatura flag:

Teorema 1.6.3. [9] Seja F uma métrica de Finsler com curvatura flag escalar sobre uma variedade $M$. Suponha que a $S$-curvatura é quase isotrópica, $S=(n+1) c F+\eta$, onde $c=c(x)$ é uma função escalar e $\eta=\eta_{i} y^{i}$ é uma 1-forma fechada, então a curvatura flag é quase isotrópica, e é dada por:

$$
K=\frac{3 c_{x^{m}} y^{m}}{F}+\sigma
$$

onde $\left(x^{m}, y^{m}\right)$ são coordenadas locais de $T M$ e $\sigma=\sigma(x)$ é uma função escalar de $M$.

\subsection{Métricas esfericamente Simétricas}

Vamos denotar por $M_{s}^{n}$ um subconjunto de $\mathbb{R}^{n}$ aberto, conexo e simétrico com respeito à origem, isto é: $M_{s}^{n}$ é uma bola aberta $\mathbb{B}(\nu)$ de raio $\nu$ centrado na origem, ou a região anular $\mathbb{B}\left(\nu_{1}\right) \backslash \mathbb{B}\left(\nu_{2}\right), \nu_{1}>\nu_{2} \geq 0$, ou $\mathbb{R}^{n}$.

Uma métrica de Finsler $F$ sobre $M_{s}^{n} \subset \mathbb{R}^{n}$ é chamada esfericamente simétrica se satisfaz,

$$
F(A x, A y)=F(x, y)
$$


para todo $x \in M, y \in T_{x} M_{s}^{n}$ e $A \in O(n)$. Zhou; Huang e Mo, obtiveram o seguinte resultado, cuja demonstração será incluída por ser simples e o resultado será fundamental nos seguintes capítulos.

Lema 1.7.1. [42] [15] Uma métrica de Finsler $F$ sobre um espaço $M_{s}^{n} \in \mathbb{R}^{n}$ é esfericamente simétrica se, e somente se, existe uma função $\phi: I \times J \subset \mathbb{R} \times \mathbb{R} \rightarrow \mathbb{R}$ tal que

$$
F(x, y)=|y| \phi\left(|x|, \frac{\langle x, y\rangle}{|y|}\right)
$$

onde $(x, y) \in \mathcal{T} M_{s}^{n}:=T M_{s}^{n} \backslash\{0\}$.

Demonstração. Assuma que

$$
F(x, y)=|y| \phi\left(|x|, \frac{<x, y>}{|y|}\right)
$$

para alguma função $\phi:[0, r) \times \mathbb{R} \rightarrow \mathbb{R}$. É fácil ver que

$$
<A x, A y>=<x, A^{\top} A y>=<x, y>
$$

para $x, y \in \mathbb{R}^{n}$ e $A \in O(n)$. Em particular, temos $|A x|=|x|$ para $x \in \mathbb{R}^{n}$. Assim,

$$
F(A x, A y)=|A x| \phi\left(|A x|, \frac{<A x, A y>}{|A y|}\right)=|y| \phi\left(|x|, \frac{<x, y>}{y}\right)=F(x, y) .
$$

Por outro lado, suponha que $F$ é esfericamente simétrica. Denote a base ortonormal de $\mathbb{R}^{n}$ por $e_{1}, \ldots, e_{n}$, onde

$$
e_{j}=(0, \ldots, 0,1,0, \ldots, 0), \quad j=1, \ldots, n .
$$

Para $x \neq 0$, denotemos

$$
\epsilon_{1}=\frac{x}{|x|}, \quad \epsilon_{2}=\frac{y-\frac{<x, y>}{|x|^{2}} x}{\left|y-\frac{\langle x, y>}{|x|^{2}} x\right|}
$$

Então $\epsilon_{1}$ e $\epsilon_{2}$ são vetores ortonormais em $\mathbb{R}^{n}$. Isto mostra que existe $A \in O(n)$ tal que

$$
A \epsilon_{1}=e_{1}, \quad A \epsilon_{2}=e_{2} .
$$

Observe que

$$
A x=A\left(|x| \epsilon_{1}\right)=|x| A \epsilon_{1}=|x| e_{1}
$$


e

$$
\begin{aligned}
A y & =A\left(\left|y-\frac{<x, y>}{|x|^{2}} x\right| \epsilon_{2}+\frac{<x, y>}{|x|^{2}} x\right) \\
& =A\left(\frac{<x, y>}{|x|^{2}} x+\frac{\sqrt{|x|^{2}|y|^{2}-<x, y>^{2}}}{|x|} \epsilon_{2}\right) \\
& =\frac{<x, y>}{|x|^{2}} A x+\frac{\sqrt{|x|^{2}|y|^{2}-<x, y>^{2}}}{|x|} A \epsilon_{2} \\
& =\frac{<x, y>}{|x|} e_{1}+\frac{\sqrt{|x|^{2}|y|^{2}-<x, y>^{2}}}{|x|} e_{2} .
\end{aligned}
$$

Observe que se $x=0$, então $F(x, y)=\psi(|y|)$.

Aplicando a simetria de $F$, obtemos

$$
\begin{aligned}
F(x, y) & =F(A x, A y) \\
& =F\left(|x| e_{1}, \frac{<x, y>}{|x|} e_{1}+\frac{\sqrt{|x|^{2}|y|^{2}-<x, y>^{2}}}{|x|} e_{2}\right) \\
& =F\left(|x|, 0, \ldots, 0 ; \frac{<x, y>}{|x|}, \frac{\sqrt{|x|^{2}|y|^{2}-<x, y>^{2}}}{|x|}, 0, \ldots, 0\right) \\
& =\psi(|x|,<x, y>,|y|),
\end{aligned}
$$

onde $\psi:[0, r) \times \mathbb{R}^{2} \rightarrow \mathbb{R}$. Sendo $F$ positiva homogênea de grau 1 com respeito a $y$, temos

$$
\begin{aligned}
\lambda \psi(|x|,<x, y>,|y|) & =\lambda F(x, y) \\
& =F(x, \lambda y) \\
& =\psi(|x|,<x, \lambda y>,|\lambda y|) \\
& =\psi(|x|, \lambda<x, y>, \lambda|y|),
\end{aligned}
$$

para $\lambda \in[0,+\infty)$. Em particular, temos

$$
\frac{1}{|y|} \psi(|x|,<x, y>,|y|)=\psi\left(|x|, \frac{<x, y>}{|y|}, 1\right):=\phi\left(|x|, \frac{<x, y>}{|y|}\right),
$$

onde $y \in T_{x} M_{s}^{n} \backslash\{0\}$ e $\phi: I \times J \rightarrow \mathbb{R}$. 
O seguinte resultado é um caso particular do que foi provado para $(\alpha, \beta)$-métricas independentemente em [14], [24] e [40]. Incluiremos a demonstração apenas no caso particular das métricas de Finsler esfericamente simétricas.

Denotaremos por $\phi_{r}$ e $\phi_{s}$ as derivadas parciais de $\phi$ com respeito a primeira e segunda coordenadas de $\phi(r, s)$ respectivamente.

Proposição 1.7.1. Seja $F=|y| \phi\left(|x|, \frac{\langle x, y\rangle}{|y|}\right)$ uma métrica de Finsler esfericamente simétrica sobre $M_{s}^{n} \subset \mathbb{R}^{n}$. Sejam $x^{1}, \ldots, x^{n}$ as coordenadas sobre $\mathbb{R}^{n}$ e $y=\sum y^{i} \partial / \partial x_{i}$. Então os coeficientes geodésicos são dados por

$$
G^{i}=|y| P y^{i}+|y|^{2} Q x^{i}
$$

onde

$$
Q:=\frac{1}{2 r} \frac{r \phi_{s s}-\phi_{r}+s \phi_{r s}}{\phi-s \phi_{s}+\left(r^{2}-s^{2}\right) \phi_{s s}}, \quad r:=|x|, \quad s:=\frac{\langle x, y\rangle}{|y|}
$$

$e$

$$
P:=\frac{r \phi_{s}+s \phi_{r}}{2 r \phi}-\frac{Q}{\phi}\left[s \phi+\left(r^{2}-s^{2}\right) \phi_{s}\right]
$$

Demonstração. De fato, vamos mostrar que (1.48) é equivalente a (1.12). Para isso precisamos das seguintes derivadas parciais com respeito as coordenadas de $y=\left(y^{i}\right)$ :

$$
\begin{aligned}
F_{y^{l}} & =\phi \frac{y^{l}}{|y|}+|y| \phi_{s} s_{y^{l}} \\
& =\phi \frac{y^{l}}{|y|}+|y| \phi_{s}\left\{-\frac{s y^{l}}{|y|^{2}}+\frac{x^{l}}{|y|}\right\} \\
& =\phi \frac{y^{l}}{|y|}-\frac{s}{|y|} \phi_{s} y^{l}+\phi_{s} x^{l}
\end{aligned}
$$

onde usamos que $s_{y^{j}}=-\frac{s y^{j}}{|y|^{2}}+\frac{x^{j}}{|y|}$. De (1.51) temos que,

$$
F F_{y^{l}}=\left(\phi^{2}-s \phi \phi_{s}\right) y^{l}+|y| \phi \phi_{s} x^{l}
$$

Agora vamos calcular as derivadas de $F^{2}$ com respeito a $y^{l}$

$$
\begin{aligned}
{\left[\frac{1}{2} F^{2}\right]_{y^{l}} } & =F F_{y^{l}} \\
& =y^{l} \phi^{2}-\phi \phi_{s}\left\{s y^{l}-|y| x^{l}\right\} .
\end{aligned}
$$

Derivando novamente com respeito a $y^{i}$

$$
\begin{aligned}
{\left[\frac{1}{2} F^{2}\right]_{y^{l} y^{i}}=} & \phi^{2} \delta^{l i}+2 \phi \phi_{s}\left\{-\frac{s y^{i}}{|y|^{2}}+\frac{x^{i}}{|y|}\right\} y^{l}+\left\{\phi_{s}^{2} s_{y^{i}}+\phi \phi_{s s} x^{i}\right\}\left\{|y| x^{l}-s y^{l}\right\} \\
& +\phi \phi_{s}\left\{\frac{y^{i} x^{l}}{|y|}-s_{y^{i}} y^{l}-s \delta^{l i}\right\}
\end{aligned}
$$


tendo em conta que $|y| . s=<x, y>$ e multiplicando (1.54) por $|y|$ e $x^{i}$ e fazendo a somatória em $i$, temos

$$
\begin{aligned}
x^{i}|y|\left[\frac{1}{2} F^{2}\right]_{y^{l} y^{i}}= & |y| \phi^{2} x^{l}+2|y| \phi \phi_{s}\left\{-\frac{s^{2}}{|y|}+\frac{|x|^{2}}{|y|}\right\} y^{l}+|y|\left\{\phi_{s}^{2}+\phi \phi_{s s}\right\}\left\{|y| x^{l}-s y^{l}\right\}\left\{-\frac{s^{2}}{|y|}+\frac{|x|^{2}}{|y|}\right\} \\
& +|y| \phi \phi_{s}\left\{s x^{l}-\left(-\frac{s^{2}}{|y|}+\frac{|x|^{2}}{|y|}\right) y^{l}-s x^{l}\right\} \\
= & |y|\left\{\phi^{2}+\left(\phi_{s}^{2}+\phi \phi_{s s}\right)\left(-s^{2}+r^{2}\right)\right\} x^{l} \\
& +|y|\left\{\frac{2}{|y|} \phi \phi_{s}\left(r^{2}-s^{2}\right)-\frac{s}{|y|}\left(\phi_{s}^{2}+\phi \phi_{s s}\right)\left(r^{2}-s^{2}\right)-\frac{1}{|y|} \phi \phi_{s}\left(r^{2}-s^{2}\right)\right\} y^{l} \\
= & |y|\left\{\phi^{2}+\left(\phi_{s}^{2}+\phi \phi_{s s}\right)\left(r^{2}-s^{2}\right)\right\} x^{l}+\left\{\phi \phi_{s}-s \phi_{s}^{2}-s \phi \phi_{s s}\right\}\left(r^{2}-s^{2}\right) y^{l} .
\end{aligned}
$$

Também precisamos das derivadas de $F_{x^{k}}, F_{x^{k}} y^{l}$

$$
\begin{aligned}
F_{x^{k}} & =|y|\left\{\frac{x^{k}}{r} \phi_{r}+\frac{y^{k}}{|y|} \phi_{s}\right\}, \\
F_{x^{k} y^{l}} & =\left\{\phi_{r} \frac{x^{k}}{|x|}+\phi_{s} \frac{y^{k}}{|y|}\right\} \frac{y^{l}}{|y|}+|y|\left\{\phi_{r s} s_{y^{l}} \frac{x^{k}}{|x|}+\phi_{s s} s_{y^{l}} \frac{y^{k}}{|y|}+\phi_{s}\left(\frac{\delta_{l}^{k}}{|y|}-\frac{y^{k} y^{l}}{|y|^{3}}\right)\right\},
\end{aligned}
$$

para obter $y^{k} F_{x^{k}}$ e $F_{x^{k} y^{l}} y^{k}$

$$
\begin{aligned}
y^{k} F_{x^{k}} & =\frac{|y|^{2}}{r}\left\{s \phi_{r}+r \phi_{s}\right\}, \\
F_{x^{k} y^{l}} y^{k} & =\left\{s \phi_{r} \frac{|y|}{|x|}+\phi_{s}|y|\right\} \frac{y^{l}}{|y|}+|y|\left\{s \phi_{r s} s_{y^{l}} \frac{|y|}{|x|}+s|y| \phi_{s s} s_{y^{l}}+\phi_{s}\left(\frac{y^{l}}{|y|}-\frac{y^{l}}{|y|}\right)\right\} \\
& =\left\{\phi_{r} \frac{s}{|x|}+\phi_{s}\right\} y^{l}+|y|\left\{\phi_{r s} s \frac{|y|}{|x|}+|y| \phi_{s s}\right\} s_{y^{l}} .
\end{aligned}
$$

Temos então, de (1.59) e (1.56) que

$$
\frac{\phi}{2}\left[F_{x^{k} y^{l}} y^{k}-F_{x^{l}}\right]=\frac{\phi}{2 r}\left[\phi_{r}-s \phi_{r s}-r \phi_{s s}\right]\left[s y^{l}-|y| x^{l}\right],
$$

e de (1.52) temos ainda

$$
\begin{aligned}
-\frac{1}{\phi}\left[s \phi+\left(r^{2}-s^{2}\right) \phi_{s}\right] F F_{y^{l}} & =-\left[s \phi+\left(r^{2}-s^{2}\right) \phi_{s}\right]\left[\phi y^{l}-s \phi_{s} y^{l}+|y| \phi_{s} x^{l}\right] \\
& =-\left[\phi-s \phi_{s}\right]\left[s \phi+\left(r^{2}-s^{2}\right) \phi_{s}\right] y^{l}+|y| \phi_{s}\left[s \phi+\left(r^{2}-s^{2}\right) \phi_{s}\right] .
\end{aligned}
$$

Observe que só precisamos mostrar o seguinte,

$$
-\frac{Q}{\phi}\left[s \phi+\left(r^{2}-s^{2}\right) \phi_{s}\right] g_{i l} y^{i}+|y| Q g_{i l} x^{i}=\frac{\phi}{2}\left[F_{x^{k} y^{l}} y^{k}-F_{x^{l}}\right] \text {. }
$$


Segue da identidade $F F_{y^{l}}=g_{i l} y^{i}$, e de somar (1.55) e (1.61), que

$$
\begin{aligned}
u g_{l i} x^{i}-\frac{1}{\phi}\left[s \phi+\left(r^{2}-s^{2}\right) \phi_{s}\right] F F_{y^{l}}= & |y|\left[\phi^{2}+\left(\phi_{s}^{2}+\phi \phi_{s s}\right)\left(r^{2}-s^{2}\right)-\phi_{s}\left(s \phi+\left(r^{2}-s^{2}\right) \phi_{s}\right)\right] x^{l} \\
& +\left[\left(\phi \phi_{s}-s \phi_{s}^{2}-s \phi \phi_{s s}\right)\left(r^{2}-s^{2}\right)-\left(s \phi+\left(r^{2}-s^{2}\right) \phi_{s}\right)\left(\phi-s \phi_{s}\right)\right] y^{l} \\
= & |y|\left[\phi^{2}+\left(r^{2}-s^{2}\right) \phi \phi_{s s}-s \phi \phi_{s}\right] x^{l} \\
& -\left[s\left(s^{2}-s^{2}\right) \phi \phi_{s s}+s \phi\left(\phi-s \phi_{s}\right)\right] y^{l} \\
= & \phi\left[\phi-s \phi_{s}+\left(r^{2}-s^{2}\right) \phi_{s s}\right]\left(u x^{l}-s y^{l}\right) .
\end{aligned}
$$

Por outro lado, dividindo (1.60) por $Q$, temos

$$
\frac{1}{Q} \frac{\phi}{2}\left[F_{x^{k} y^{l}} y^{k}-F_{x^{l}}\right]=-\phi\left[\phi-s \phi_{s}+\left(r^{2}-s^{2}\right) \phi_{s s}\right]\left(s y^{l}-|y| x^{l}\right) .
$$

Igualando (1.63) com (1.64) obtemos (1.62) o que finaliza a demonstração.

C.Yu e H. Zhu, [40] deram as condições necessárias e suficientes para que uma $(\alpha, \beta)$-métrica generalizada seja uma métrica de Finsler (ver Proposição 1.2.2). Em particular considerando $F(x, y)=|y| \phi\left(|x|, \frac{\langle x, y>}{|y|}\right)$, temos que $F$ é uma métrica de Finsler se, e somente se, a função $\phi>0$ satisfaz:

$$
\phi(s)-s \phi_{s}(s)+\left(r^{2}-s^{2}\right) \phi_{s s}(s)>0, \quad \text { quando } n \geq 2,
$$

com a condição adicional

$$
\phi(s)-s \phi_{s}(s)>0, \quad \text { quando } n \geq 3 \text {. }
$$

\subsection{Equação de Transporte}

Nesta seção, vamos dar noções da equação de transporte e o método de solução que será utilizado neste trabalho.

Seja $\psi=\psi(r, s)$ uma função diferenciável, a seguinte equação é chamada equação de transporte

$$
\psi_{r}(r, s)+v(r, s) \psi_{s}(r, s)=T(r, s, \psi(r, s)),
$$

onde $v(r, s)$ e $T(r, s, \psi(r, s))$ são diferenciáveis. Observe que esta equação pode ser escrita como $\vec{v} \cdot \operatorname{grad} \psi=P$, onde $\vec{v}=(1, v)$ e $\operatorname{grad} \psi=\left(\psi_{r}, \psi_{s}\right)$.

Isto motiva a seguinte interpretação geométrica: estamos procurando uma superfície $z=\psi(r, s)$ cuja derivada direcional com respeito ao vetor $\vec{v}$ é $T(r, s, \psi)$. Esta interpretação geométrica é a base para o seguinte método de resolução de (1.67). 
As curvas $(r, X(r))$ no plano $(r, s)$ que são tangentes ao campo vetorial $(1, v)$ são chamadas curvas características.

Dessa definição podemos dizer que a curva característica que passa por $(r, s)=\left(r_{0}, c\right)$ é o gráfico da função $X$ que satisfaz a equação diferencial ordinária:

$$
\frac{d X}{d r}=v(r, X(r))
$$

com a condição inicial $X\left(r_{0}\right)=c$.

Denotemos o valor de $\psi$, ao longo da curva característica $(r, X(r))$, por $\Psi(r)=$ $\psi(r, X(r))$, então temos

$$
\frac{d}{d r} \Psi=\frac{\partial \psi}{\partial r}+\frac{\partial \psi}{\partial s} \frac{d X}{d r}=\psi_{r}+v \psi_{s}=T
$$

Por isso, o valor de $\psi$ ao longo da curva característica está determinado pela seguinte equação diferencial ordinária:

$$
\Psi^{\prime}=T(r, X(r), \Psi(r))
$$

A equação (1.70), com valor inicial $\Psi\left(r_{0}\right)=\psi_{r_{0}}(c)$ determina o valor de $\psi$ ao longo da curva característica que intercepta o eixo $\left(r_{0}, s\right)$ em $\left(r_{0}, c\right)$, pois

$$
\Psi\left(r_{0}\right)=\psi\left(r_{0}, X\left(r_{0}\right)\right)=\psi\left(r_{0}, c\right)=\psi_{r_{0}}(c) .
$$

A superfície $z=\psi(r, s)$ é a coleção (o recobrimento) das curvas obtidas fazendo $c$ assumir todos os valores reais.

Exemplo 1.8.1. Considere a equação diferencial parcial:

$$
u_{r}+s u_{s}=-u \text {. }
$$

A equação diferencial ordinária característica é $S^{\prime}=S$. A solução desta equação satisfazendo a condição inicial $S(0)=k$ é $S=k e^{r}$. Então a curva característica que intercepta o eixo $x$ no ponto $(k, 0)$ é dada por $k=s e^{-r}$.

A equação diferencial ordinária que descreve o valor de $u$ ao longo da curva característica é

$$
U^{\prime}=-U
$$

A solução desta equação satisfazendo a condição inicial $U(0)=u_{0}(k)$ é

$$
U(r)=u_{0}(k) e^{-r}
$$

Finalmente obtemos a solução geral de (1.71):

$$
u(r, s)=u_{0}\left(s e^{-r}\right) e^{-r} .
$$


Os seguintes lemas são aplicações das curvas características, os quais serão de muita importância nos resultados dos capítulos subsequentes.

Lema 1.8.1. Sejam $f=f(r)$ e $g=g(r)$ funções, tais que as seguintes expressões

$$
\int 2 r\left(2 g+r^{2} f\right) d r \quad e \quad \int 2 r f e^{\int 2 r\left(2 g+r^{2} f\right) d r} d r
$$

estejam bem definidas. Então

$$
\left[1-\left(r^{2}-s^{2}\right)\left(2 g+s^{2} f\right)\right] r \psi_{s}(r, s)+s \psi_{r}(r, s)=0
$$

é equivalente a

$$
\psi(r, s)=\eta(\varphi(r, s)))
$$

onde $\eta$ é qualquer função diferenciável, e

$$
\varphi(r, s)=\frac{r^{2}-s^{2}}{\left(r^{2}-s^{2}\right) \int 2 r f e^{\int 2 r\left(2 g+r^{2} f\right) d r} d r-e^{\int 2 r\left(2 g+r^{2} f\right) d r}} .
$$

Demonstração. Para $s \neq 0$, observe que (1.73) é equivalente à seguinte equação de transporte

$$
\begin{aligned}
& \psi_{r}+v(r, s) \psi_{s}=0, \\
& \psi\left(r_{0}, s\right)=\psi_{r_{0}}(s),
\end{aligned}
$$

onde

$$
v(r, s)=\frac{r}{s}\left[1-\left(r^{2}-s^{2}\right)\left(2 g(r)+f(r) s^{2}\right)\right] .
$$

Consideremos a curva característica $(r, X(r))$ desta equação. Primeiro resolveremos a equação dada por (1.68) que torna-se,

$$
X^{\prime}(r)=\frac{r}{X(r)}\left[1-\left(r^{2}-X^{2}(r)\right)\left(2 g(r)+f(r) X^{2}(r)\right)\right]
$$

podendo ser reescrito como

$$
2 X(r) X^{\prime}(r)-2 r=2 r\left(X^{2}(r)-r^{2}\right)\left(2 g(r)+f(r) X^{2}(r)\right) .
$$

Defina-se $\varsigma(r)=X^{2}(r)-r^{2}$, assim a última expressão (1.80) pode ser vista como

$$
\varsigma^{\prime}=2 r\left(2 g+r^{2} f\right) \varsigma+2 r f \varsigma^{2}
$$

que é uma equação do tipo Ricatti, que tem como solução geral

$$
\varsigma=\frac{e^{\int 2 r\left(2 g+r^{2} f\right) d r}}{c_{0}-\int 2 r f e^{\int 2 r\left(2 g+r^{2} f\right) d r} d r}, \quad c_{0} \in \mathbb{R} .
$$


Então, a solução da equação (1.79) com a condição inicial $X\left(r_{0}\right)=c$ é dada por:

$$
X(r)=\sqrt{r^{2}+\frac{e^{\int_{r_{0}} 2 r\left(2 g+r^{2} f\right) d r}}{\frac{1}{c^{2}-r_{0}^{2}}-\int_{r_{0}} 2 r f e^{\int_{r_{0}} 2 r\left(2 g+r^{2} f\right) d r} d r}},
$$

que é equivalente a

$$
\left(X^{2}(r)-r^{2}\right)^{-1}=\frac{\frac{1}{c^{2}-r_{0}^{2}}-\int_{r_{0}} 2 r f e^{\int_{r_{0}} 2 r\left(2 g+r^{2} f\right) d r}}{e^{\int_{r_{0}} 2 r\left(2 g+r^{2} f\right) d r}},
$$

e que pode ser melhorada por

$$
\frac{1}{c^{2}-r_{0}^{2}}=\int_{r_{0}} 2 r f e^{\int_{r_{0}} 2 r\left(2 g+r^{2} f\right) d r}+\left(X^{2}(r)-r^{2}\right)^{-1} e^{\int_{r_{0}} 2 r\left(2 g+r^{2} f\right) d r} .
$$

Assim, para $r^{2}-s^{2}>0$, a curva característica que intersecta o eixo $\left(r_{0}, s\right)$ no ponto $\left(r_{0}, c\right)$ está determinada por

$$
\begin{aligned}
c & =\sqrt{r_{0}^{2}+\left[\int_{r_{0}} 2 r f e^{\int_{r_{0}} 2 r\left(2 g+r^{2} f\right) d r} d r-\frac{e^{\int_{r_{0}} 2 r\left(2 g+r^{2} f\right) d r}}{r^{2}-s^{2}}\right]^{-1}} \\
& =\sqrt{r_{0}^{2}+\frac{r^{2}-s^{2}}{\left(r^{2}-s^{2}\right) \int_{r_{0}} 2 r f e^{\int_{r_{0}} 2 r\left(2 g+r^{2} f\right) d r} d r-e^{\int 2 r\left(2 g+r^{2} f\right) d r}}}
\end{aligned}
$$

A equação (1.70) torna-se

$$
\Psi^{\prime}(r)=0
$$

com condição inicial $\Psi\left(r_{0}\right)=\psi_{r_{0}}(c)$, que tem como solução geral

$$
\Psi=\psi_{r_{0}}(c) .
$$

Assim, a solução geral do problema de valor inicial (1.76)-(1.77) é dada por

$$
\psi(r, s)=\psi_{r_{0}}\left(\sqrt{r_{0}^{2}+\frac{r^{2}-s^{2}}{\left(r^{2}-s^{2}\right) \int_{r_{0}} 2 r f e^{\int_{r_{0}} 2 r\left(2 g+r^{2} f\right) d r} d r-e^{\int 2 r\left(2 g+r^{2} f\right) d r}}}\right) .
$$

Note que, $\varphi$ dada por (1.75) é uma solução da equação (1.73). Portanto, qualquer função diferenciável $\eta$ de (1.75) é a solução geral da equação (1.73).

Lema 1.8.2. Dados $f=f(r), g=g(r)$ e $L(r)$ funções diferenciáveis, então

$$
\left[1-\left(r^{2}-s^{2}\right)\left(2 g+s^{2} f\right)\right] r \psi_{s}(r, s)+s \psi_{r}(r, s)+s L(r) \psi=0
$$

é equivalente a

$$
\left.\psi(r, s)=e^{-\int L(r) d r} \eta(\varphi(r, s))\right) .
$$

onde $\eta$ é qualquer função diferenciável $\varphi$ definida em (1.75). 
Demonstração. Pelo Lema 1.8.1

$$
e^{\int L(r) d r} \psi=\eta(\varphi(r, s))
$$

é equivalente a

$$
\left[1-\left(r^{2}-s^{2}\right)\left(2 g+s^{2} f\right)\right] r\left(e^{\int L(r) d r} \psi(r, s)\right)_{s}+s\left(e^{\int L(r) d r} \psi(r, s)\right)_{r}=0
$$

isto é,

$$
e^{\int L(r) d r}\left\{s L(r) \psi(r, s)+\left[1-\left(r^{2}-s^{2}\right)\left(2 g+s^{2} f\right)\right] r \psi_{s}(r, s)+s \psi_{r}(r, s)\right\}=0 .
$$

Multiplicando por $e^{-\int L(r) d r}$ obtemos o resultado. 


\section{MÉTRICAS DE DOUGLAS}

Exemplos e resultados interessantes de métricas de Douglas podem ser vistos em [8], [17], [18], [28], [34] e [38]. No presente capítulo obteremos todas as métricas de Finsler esfericamente simétricas do tipo Douglas e apresentaremos novos exemplos de famílias de tais métricas. No entanto, nos capítulos seguintes serão apresentados mais exemplos com a condição adicional da $S$-curvatura ser nula.

\subsection{Métricas de Douglas Esfericamente Simétricas}

Antes de mostrar os teoremas principais desta seção (Teoremas 2.2.1 e 2.2.2) precisamos mostrar os lemas a seguir. Para os quais requeremos as seguintes notações e identidades.

Sejam duas funções $U=U(y)$ e $V=V(y)$, definidas sobre $\mathbb{R}^{n}$. Para simplificar os cálculos, os índices inferiores representam as derivadas parciais em $y$, isto é, denotaremos por $U_{i}, U_{i j}, U_{i j k}, \ldots$ as derivadas parciais em relação a $y^{i}, y^{i} y^{j}, y^{i} y^{j} y^{k}, \ldots$, respectivamente. Também faremos uso das seguintes notações:

$\overrightarrow{j k}$ indica permutação dos índices $j$ e $k$,

$\overrightarrow{j k l}$ indica permutação dos índices $j, k, l$ na ordem $j k l, k l j, l j k$.

Assim, por exemplo, obtemos a terceira derivada com respeito a $y^{j} y^{k} y^{l}$ do produto de duas funções $U(y)$ e $V(y)$ :

$$
\begin{aligned}
(U V)_{j k l} & =\left(U_{j} V+U V_{j}\right)_{k l} \\
& =\left(U_{j k} V+U_{j} V_{k}+U_{k} V_{j}+U V_{j k}\right)_{l} \\
& =U_{j k l} V+U_{j k} V_{l}+U_{j l} V_{k}+U_{j} V_{k l}+U_{k l} V_{j}+U_{k} V_{j l}+U_{l} V_{j k}+U V_{j k l} \\
& =U V_{j k l}+\left(U_{j} V_{k l}\right)_{\overrightarrow{j k l}}+\left(U_{j k} V_{l}\right)_{\overrightarrow{j k l}}+U_{j k l} V .
\end{aligned}
$$

Sejam $u=|y|, s=\frac{<x, y>}{|y|}$ e $T:=T(s)$ uma função de $s$, então:

$$
\begin{aligned}
(T)_{j} & =T_{s} s_{j} \\
(T)_{j k} & =T_{s s} s_{j} s_{k}+T_{s} s_{j k}, \\
(T)_{j k l} & =T_{s s s} s_{j} s_{k} s_{l}+T_{s s}\left(s_{j k} s_{l}+s_{k} s_{j l}\right)+T_{s s} s_{j} s_{l k}+T_{s} s_{j k l} \\
& =T_{s s s} s_{j} s_{k} s_{l}+T_{s s}\left(s_{j k} s_{l}\right)_{\overrightarrow{j k l}}+T_{s} s_{j k l},
\end{aligned}
$$

onde os índices inferiores de $s_{j}$ e $s_{l k}$ indicam derivação com respeito a $y^{j}$ e $y^{k} y^{l}$ respectiva- 
mente. Agora calculemos as derivadas de $u=|y|$

$$
\begin{aligned}
u_{j} & =\frac{y^{j}}{u}, \\
u_{j k} & =-\frac{y^{j} y^{k}}{u^{3}}+\frac{\delta_{k}^{j}}{u}, \\
u_{j k l} & =\frac{3 y^{j} y^{k} y^{l}}{u^{5}}-\frac{\left(y^{j} \delta_{l}^{k}+y^{k} \delta_{l}^{j}+y^{l} \delta_{k}^{j}\right)}{u^{3}} \\
& =\frac{3 y^{j} y^{k} y^{l}}{u^{5}}-\frac{\left(y^{j} \delta_{l}^{k}\right) \overrightarrow{j k l}}{u^{3}} .
\end{aligned}
$$

Da mesma forma para $1 / u$

$$
\begin{aligned}
\left(\frac{1}{u}\right)_{j} & =-\frac{y^{j}}{u^{3}} \\
\left(\frac{1}{u}\right)_{j k} & =\frac{1}{u^{3}}\left(\frac{3 y^{j} y^{k}}{u^{3}}-\delta_{j k}\right), \\
\left(\frac{1}{u}\right)_{j k l} & =-\frac{15 y^{j} y^{k} y^{l}}{u^{7}}+\frac{3\left(y^{k} \delta_{l j}\right)+3 \delta_{j k} y^{l}+3 \delta_{k l} y^{j}}{u^{5}} \\
& =\frac{3}{u^{5}}\left[\left(y^{j} \delta_{k l}\right)_{\overrightarrow{j k l}}-\frac{5}{u^{2}} y^{j} y^{k} y^{l}\right] .
\end{aligned}
$$

Temos ainda as derivadas de $s=\frac{\langle x, y>}{|y|}$

$$
\begin{aligned}
s_{j} & =-\frac{s y^{j}}{u^{2}}+\frac{x^{j}}{u} \\
& =\frac{1}{u}\left[x^{j}-\frac{s y^{j}}{u}\right], \\
s_{j k} & =\frac{1}{u^{2}}\left[\frac{3 s}{u^{2}} y^{j} y^{k}-s \delta_{j k}-\frac{x^{k} y^{j}+x^{j} y^{k}}{u}\right], \\
s_{j k l} & =\frac{1}{u}\left[\left(x^{j}\left(\frac{3 y^{k} y^{l}}{u^{2}}-\delta_{k l}\right)\right)_{\overrightarrow{j k l}}+\frac{2 s}{u}\left(\left(y^{j} \delta_{k l}\right)_{\overrightarrow{j k l}}-\frac{5 y^{j} y^{k} y^{l}}{u^{2}}\right)\right] .
\end{aligned}
$$


Logo, de (2.11)-(2.13) temos

$$
\begin{gathered}
s_{j} s_{k}=\frac{1}{u^{2}}\left[x^{j} x^{k}-\frac{s}{u}\left(x^{j} y^{k}+x^{k} y^{j}\right)+\frac{s^{2}}{u^{2}} y^{j} y^{k}\right], \\
s_{j} s_{k} s_{l}=\frac{1}{u^{3}}\left[x^{j} x^{k} x^{l}-\frac{s}{u}\left(x^{j} x^{k} y^{l}\right)_{\overrightarrow{j k l}}+\frac{s^{2}}{u^{2}}\left(x^{j} y^{k} y^{l}\right)_{\overrightarrow{j k l}}-\frac{s^{3}}{u^{3}} y^{j} y^{k} y^{l}\right], \\
s_{j k} s_{l}=\frac{1}{u^{3}}\left[\frac{3 s}{u^{2}} x^{l} y^{j} y^{k}-\frac{3 s^{2}}{u^{3}} y^{j} y^{k} y^{l}-s \delta_{j k} x^{l}+\frac{s^{2}}{u} \delta_{j k} y^{l}\right. \\
\left.\quad-\frac{x^{k} y^{j}+x^{j} y^{k}}{u} x^{l}+\frac{s}{u^{2}}\left(x^{k} y^{j}+x^{j} y^{k}\right) y^{l}\right] .
\end{gathered}
$$

Agora podemos mostrar a seguinte Proposição.

Proposição 2.1.1. Seja $F=|y| \phi\left(|x|, \frac{\langle x, y\rangle}{|y|}\right)$ uma métrica esfericamente simétrica sobre $M_{s}^{n} \subset \mathbb{R}^{n}$. Então a curvatura de Douglas de $F$ é dada por

$$
\begin{aligned}
D_{j k l}^{i}= & \frac{1}{u} R_{s s}\left(\delta_{j}^{i} x^{k} x^{l}\right)_{\overrightarrow{j k l}}+\frac{1}{u}\left(R-s R_{s}\right)\left(\delta_{j}^{i} \delta_{k l}\right)_{\overrightarrow{j k l}} \\
& -\frac{s}{u^{2}} R_{s s}\left(\delta_{j}^{i}\left(x^{k} y^{l}+x^{l} y^{k}\right)\right)_{\overrightarrow{j k l}} \\
& -\frac{s}{u^{2}} R_{s s} y^{i}\left(\delta_{j k} x^{l}\right)_{\overrightarrow{j k l}}+\frac{1}{u}\left(Q_{s}-s Q_{s s}\right) x^{i}\left(\delta_{j k} x^{l}\right)_{\overrightarrow{j k l}} \\
& +\frac{1}{u^{3}}\left(s^{2} R_{s s}+s R_{s}-R\right)\left(\delta_{j}^{i} y^{k} y^{l}\right)_{\overrightarrow{j k l}} \\
& +\frac{1}{u^{3}}\left(s^{2} R_{s s}+s R_{s}-R\right) y^{i}\left(\delta_{j k} y^{l}\right)_{\overrightarrow{j k l}} \\
& +\frac{1}{u^{5}}\left(3 R-3 s R_{s}-6 s^{2} R_{s s}-s^{3} R_{s s s}\right) y^{i} y^{j} y^{k} y^{l} \\
& +\frac{1}{u^{4}}\left(s^{2} R_{s s s}+3 s R_{s s}\right) y^{i}\left(y^{j} y^{k} x^{l}\right)_{\overrightarrow{j k l}}+\frac{1}{u^{2}} R_{s s s} y^{i} x^{j} x^{k} x^{l} \\
& -\frac{1}{u^{3}}\left(R_{s s}+s R_{s s s}\right) y^{i}\left(y^{j} x^{k} x^{l}\right)_{\overrightarrow{j k l}} \\
& +\frac{1}{u^{3}}\left(s^{2} Q_{s s s}+s Q_{s s}-Q_{s}\right)^{i}\left(x^{j} y^{k} y^{l}\right)_{\overrightarrow{j k l}} \\
& -\frac{s}{u^{2}} Q_{s s s} x^{i}\left(x^{j} x^{l} y^{k}\right)_{\overrightarrow{j k l}}+\frac{1}{u} Q_{s s s} x^{i} x^{j} x^{k} x^{l} \\
& +\frac{1}{u^{2}}\left(s^{2} Q_{s s}-s Q_{s}\right) x^{i}\left(\delta_{j k} y^{l}\right)_{\overrightarrow{j k l}} \\
& +\frac{1}{u^{4}}\left(3 s Q_{s}-3 s^{2} Q_{s s}-s^{3} Q_{s s s}\right) x^{i} y^{j} y^{k} y^{l}
\end{aligned}
$$

onde $Q$ é dado por (1.49) e

$$
R:=-\frac{1}{n+1}\left[2 s Q+\left(r^{2}-s^{2}\right) Q_{s}\right] .
$$


Demonstração. Seja $P(r, s)$ a função dada por (1.50) da Proposição 1.7.1. Tendo em conta que $|y| P(r, s)$ é homogênea de grau 1 com respeito a $y$ segue do teorema de Euler(Teorema1.1.1) que

$$
\sum_{i=1}^{n} \frac{\partial\left(u P y^{i}\right)}{\partial y^{i}}=\sum_{i=1}^{n} y^{i} \frac{\partial(u P)}{\partial y^{i}}+\sum_{i=1}^{n} u P=(n+1) u P
$$

onde $u=|y|$. Agora procedemos ao cálculo de $\sum_{i=1}^{n} \frac{\partial G^{i}}{\partial y^{i}}$, onde os $G^{i}$ são dadas por (1.48). Tendo em conta que

$$
s_{i} x^{i}=\left(-\frac{<x, y>}{|y|^{3}} y^{i}+\frac{x^{i}}{|y|}\right) x^{i}=-\frac{s^{2}}{u}+\frac{r^{2}}{u},
$$

e

$$
u u_{y^{i}} x^{i}=u s,
$$

temos

$$
\begin{aligned}
\sum_{i=1}^{n} \frac{\partial G^{i}}{\partial y^{i}} & =(n+1) u P+\sum_{i=1}^{n}\left[u^{2} Q_{y^{i}} x^{i}+2 u u_{y^{i}} Q x^{i}\right] \\
& =u\left[(n+1) P+2 s Q+\left(r^{2}-s^{2}\right) Q_{s}\right] .
\end{aligned}
$$

Logo,

$$
\begin{aligned}
\left(G^{i}-\frac{1}{n+1} \sum_{m} \frac{\partial G^{m}}{\partial y^{m}} y^{i}\right) & =u P y^{i}+u^{2} Q x^{i}-u P y^{i}-\frac{u}{n+1} Q_{s}\left(r^{2}-s^{2}\right) y^{i}-\frac{2 s u}{n+1} Q y^{i} \\
& =u R y^{i}+u^{2} Q x^{i}
\end{aligned}
$$

onde $R$ é dado por (2.18).

Portanto a curvatura de Douglas, definida por (1.28), é dada por

$$
\begin{aligned}
D_{j k l}^{i} & =\frac{\partial^{3}}{\partial y^{j} \partial y^{k} \partial y^{l}}\left(u R y^{i}+u^{2} Q x^{i}\right) \\
& =\frac{\partial^{3}}{\partial y^{j} \partial y^{k} \partial y^{l}}\left(u R y^{i}\right)+\frac{\partial^{3}}{\partial y^{j} \partial y^{k} \partial y^{l}}\left(u^{2} Q x^{i}\right) .
\end{aligned}
$$

Primeiramente calcularemos as derivadas envolvendo $R$ em (2.21) usando (2.1) e também as expressões (2.5)-(2.7).

$$
\begin{aligned}
(u R)_{j k l}= & u_{j k l} R+\left(u_{j k} R_{l}\right)_{\overrightarrow{j k l}}+u R_{j k l} \\
= & {\left[\frac{3 y^{j} y^{k} y^{l}}{u^{5}}-\frac{1}{u^{3}}\left(y^{k} \delta_{l}^{k}\right)_{\overrightarrow{j k l}}\right] R+\left(\left[-\frac{y^{j} y^{k}}{u^{3}}+\frac{\delta_{k}^{j}}{u}\right] R_{s} s_{l}\right)_{\overrightarrow{j k l}}+} \\
& +\left(\frac{y^{j}}{u}\left[R_{s s} s_{k} s_{l}+R_{s} s_{l k}\right]\right)_{\overrightarrow{j k l}}+u\left(R_{s s} s_{j} s_{k} s_{l}+R_{s s}\left(s_{j k} s_{l}\right)_{\overrightarrow{j k l}}+R_{s} s_{j k l}\right)
\end{aligned}
$$


usando (2.11)-(2.16),

$$
\begin{aligned}
(u R)_{j k l}= & {\left[\frac{3 y^{j} y^{k} y^{l}}{u^{5}}-\frac{\left(y^{j} \delta_{l}^{k}\right) \overrightarrow{j k l}}{u^{3}}\right] R+\left(\left[\frac{\delta_{j}^{k}}{u}-\frac{y^{j} y^{k}}{u^{3}}\right] \frac{R_{s}}{u}\left[x^{l}-s \frac{y^{l}}{u}\right]\right)_{\overrightarrow{j k l}} } \\
& +\left(\frac{y^{j}}{u^{3}}\left[R_{s s}\left(x^{k} x^{l}-\frac{s\left(x^{l} y^{k}+x^{k} y^{l}\right)}{u}+\frac{s^{2}}{u^{2}} y^{k} y^{l}\right)+R_{s}\left(\frac{3 s}{u^{2}} y^{l} y^{k}-s \delta_{l k}-\frac{\left(x^{k} y^{l}+x^{l} y^{k}\right)}{u}\right)\right]\right)_{\overrightarrow{j k l}} \\
& +\frac{R_{s s s}}{u^{2}}\left[x^{j} x^{k} x^{l}-\frac{s}{u}\left(x^{j} x^{k} y^{l}\right)_{\overrightarrow{j k l}}+\frac{s^{2}}{u^{2}}\left(x^{j} y^{k} y^{l}\right)_{\overrightarrow{j k l}}-\frac{s^{3}}{u^{3}} y^{j} y^{k} y^{l}\right] \\
& +\frac{R_{s s}}{u^{2}}\left(\frac{3 s}{u^{2}} x^{l} y^{j} y^{k}-\frac{3 s^{2}}{u^{3}} y^{j} y^{l} y^{k}-s \delta_{j k} x^{l}+\frac{s^{2}}{u} \delta_{j k} y^{l}-\frac{x^{k} y^{j}+x^{j} y^{k}}{u} x^{l}+s y^{l} \frac{x^{k} y^{j}+x^{j} y^{k}}{u^{2}}\right)_{\overrightarrow{j k l}} \\
& +\frac{R_{s}}{u^{2}}\left[\left(\left(\frac{3 y^{k} y^{l}}{u^{2}}-\delta_{k l}\right) x^{j}\right)_{\overrightarrow{j k l}}+\frac{3 s}{u}\left(\left(y^{j} \delta_{k l}\right)_{\overrightarrow{j k l}}-\frac{5 y^{j} y^{k} y^{l}}{u^{2}}\right)\right] .
\end{aligned}
$$

Por outro lado, usando novamente (2.11)-(2.16),

$$
\begin{aligned}
(u R)_{j k}= & u_{j k} R+\left(u_{j} R_{k}\right)_{\overrightarrow{j k}}+u R_{j k} \\
= & R\left[\frac{\delta_{k}^{j}}{u}-\frac{y^{j} y^{k}}{y^{3}}\right]+\frac{R_{s}}{u}\left(y^{j} s_{k}\right)_{\overrightarrow{j k}}+u\left(R_{s s} s_{j} s_{k}+R_{s} s_{j k}\right) \\
= & R\left[\frac{\delta_{k}^{j}}{u}-\frac{y^{j} y^{k}}{y^{3}}\right]+\frac{R_{s}}{u^{2}}\left(\left(x^{k}-\frac{s y^{k}}{u}\right) y^{j}\right)_{\overrightarrow{j k}} \\
& +\frac{R_{s s}}{u}\left[x^{j} x^{k}-\frac{s}{u} x^{j} y^{k}-\frac{s x^{k} y^{j}}{u}+\frac{s^{2}}{u^{2}} y^{j} y^{k}\right] \\
& +\frac{R_{s}}{u}\left[\frac{3 s}{u^{2}} y^{j} y^{k}-s \delta_{j k}-\frac{x^{k} y^{j}+x^{j} y^{k}}{u}\right] .
\end{aligned}
$$

Com isto, podemos obter:

$$
\begin{aligned}
\frac{\partial^{3}\left(u R y^{i}\right)}{\partial y^{j} \partial y^{k} \partial y^{l}}= & (u R)_{\overrightarrow{j k l}} y^{i}+\left((u R)_{j k} \delta_{l}^{i}\right)_{\overrightarrow{j k l}} \\
= & {\left[\frac { 1 } { u ^ { 5 } } \left\{3 r y^{j} y^{k} y^{l}+s R_{s}\left(y^{j} y^{k} y^{l}\right)_{\overrightarrow{j k l}}+s^{2} R_{s s}\left(y^{j} y^{k} y^{l}\right)_{\overrightarrow{j k l}}+3 s R_{s}\left(y^{j} y^{k} y^{l}\right)_{\overrightarrow{j k l}}\right.\right.} \\
& \left.-s^{3} R_{s s s} y^{j} y^{k} y^{l}-3 s^{2} R_{s s}\left(y^{j} y^{k} y^{l}\right)_{\overrightarrow{j k l}}-15 s R_{s} y^{j} y^{k} y^{l}\right\} \\
+ & +\frac{1}{u^{4}}\left\{-R_{s}\left(x^{l} y^{j} y^{k}\right)_{\overrightarrow{j k l}}-s R_{s s}\left(x^{j} y^{k} y^{l}\right)_{\overrightarrow{j k l}}-s R_{s s}\left(x^{j} y^{k} y^{l}\right)_{\overrightarrow{j k l}}\right. \\
& -R_{s}\left(\left(x^{k} y^{l}+x^{l} y^{k}\right) y^{j}\right)_{\overrightarrow{j k l}}+s^{2} R_{s s s}\left(x^{j} y^{k} y^{l}\right)_{\overrightarrow{j k l}}+3 s R_{s s}\left(x^{l} y^{j} y^{k}\right)_{\overrightarrow{j k l}} \\
& \left.+s R_{s s}\left(\left(x^{k} y^{j}+x^{j} y^{k}\right) y^{l}\right)_{\overrightarrow{j k l}}+3 R_{s}\left(x^{j} y^{k} y^{l}\right)_{\overrightarrow{j k l}}\right\}
\end{aligned}
$$




$$
\begin{aligned}
& +\frac{1}{u^{3}}\left\{-R\left(y^{j} \delta_{l}^{k}\right)_{\overrightarrow{j k l}}-s R_{s}\left(y^{l} \delta_{k}^{j}\right)_{\overrightarrow{j k l}}+R_{s s}\left(y^{j} x^{k} x^{l}\right)_{\overrightarrow{j k l}}-s R_{s}\left(\delta_{k l} y^{j}\right)_{\overrightarrow{j k l}}\right. \\
& -s R_{s s s}\left(x^{j} x^{l} y^{k}\right)_{\overrightarrow{j k l}}+s^{2} R_{s s}+\left(\delta_{j k} y^{l}\right)_{\overrightarrow{j k l}}-R_{s s}\left(\left(x^{k} y^{j}+x^{j} y^{k}\right) x^{l}\right)_{\overrightarrow{j k l}} \\
& \left.+3 s R_{s}\left(y^{j} \delta_{k l}\right)_{\overrightarrow{j k l}}\right\} \\
& \left.+\frac{1}{u^{2}}\left\{R_{s}\left(\delta_{k}^{j} x^{l}\right)_{\overrightarrow{j k l}}+R_{s s s} x^{j} x^{k} x^{l}-s R_{s s}\left(\delta_{j k} x^{l}\right)_{\overrightarrow{j k l}}-R_{s}\left(\delta_{k l} x^{j}\right)_{\overrightarrow{j k l}}\right\}\right] y^{i} \\
& +\frac{1}{u^{3}}\left\{-R\left(\delta_{l}^{i} y^{j} y^{k}\right)_{\overrightarrow{j k l}}-s R_{s}\left(\left(y^{j} y^{k}\right)_{\overrightarrow{j k}} \delta_{l}^{i}\right)_{\overrightarrow{j k l}}+s^{2} R_{s s}\left(y^{j} y^{k} \delta_{l}^{i}\right)_{\overrightarrow{j k l}}\right. \\
& \left.+3 s R_{s}\left(y^{j} y^{k} \delta_{l}^{i}\right) \underset{j k l}{ }\right\} \\
& +\frac{1}{u^{2}}\left\{R_{s}\left(\left(y^{j} x^{k}\right)_{\overrightarrow{j k}} \delta_{k}^{i}\right)_{\overrightarrow{j k l}}-s R_{s s}\left(\left(x^{j} y^{k}\right)_{\overrightarrow{j k}} \delta_{l}^{i}\right)_{\overrightarrow{j k l}}-R_{s}\left(\left(x^{k} y^{j}\right)_{\overrightarrow{j k}} \delta_{l}^{i}\right)_{\overrightarrow{j k l}}\right\} \\
& +\frac{1}{u}\left\{R\left(\delta_{k}^{j} \delta_{l}^{i}\right)_{\overrightarrow{j k l}}+R_{s s}\left(x^{j} x^{k} \delta_{l}^{i}\right)_{\overrightarrow{j k l}}-s R_{s}\left(\delta_{j k} \delta_{l}^{i}\right)_{\overrightarrow{j k l}}\right\} \\
& =\frac{1}{u^{5}}\left\{3 R-3 s R_{s}-6 s^{2} R_{s s}-s^{3} R_{s s s}\right\} y^{j} y^{k} y^{l} y^{i} \\
& +\frac{1}{u^{4}}\left\{3 s R_{s s}+s^{2} R_{s s s}\right\}\left(x^{j} y^{k} y^{l}\right)_{\overrightarrow{j k l}} y^{i}-\frac{1}{u^{3}}\left\{s R_{s s s}\right\}\left(x^{j} x^{l} y^{k}\right)_{\overrightarrow{j k l}} y^{i} \\
& +\frac{1}{u^{3}}\left\{-R+s R_{s}+s^{2} R_{s s}\right\}\left(\delta^{j} \delta_{k l}\right)_{\overrightarrow{j k l}} y^{i}-\frac{1}{u^{3}}\left\{R_{s s}\right\}\left(x^{j} x^{k} y^{l}\right)_{\overrightarrow{j k l}} y^{i} \\
& -\frac{1}{u^{2}}\left\{s R_{s s}\right\}\left(\delta_{j k} x^{l}\right)_{\overrightarrow{j k l}} y^{i}+\frac{1}{u^{2}} R_{s s s} x^{j} x^{k} x^{l} y^{i} \\
& +\frac{1}{u^{3}}\left\{-R\left(\delta_{l}^{i} y^{j} y^{k}\right)_{\overrightarrow{j k l}}+s R_{s}\left(y^{j} y^{k} \delta_{l}^{i}\right)_{\overrightarrow{j k l}}+s^{2} R_{s s}\left(Y^{j} y^{k} \delta_{l}^{i}\right)_{\overrightarrow{j k l}}\right\} \\
& +\frac{1}{u^{2}}\left\{2 R_{s}-s R_{s s}-2 R_{s}\right\}\left(\left(x^{k} y^{j}\right)_{\overrightarrow{j k}} \delta_{l}^{i}\right)_{\overrightarrow{j k l}} \\
& +\frac{1}{u}\left\{\left(R-s R_{s}\right)\left(\delta_{j k} \delta_{l}^{i}\right)_{\overrightarrow{j k l}}+R_{s s}\left(x^{j} x^{k} \delta_{l}^{i}\right)_{\overrightarrow{j k l}}\right\} .
\end{aligned}
$$

Simplificando a expressão anterior temos,

$$
\begin{aligned}
\frac{\partial^{3}\left(u R y^{i}\right)}{\partial y^{j} \partial y^{k} \partial y^{l}}= & \frac{1}{u}\left\{R_{s s}\left(x^{j} x^{k} \delta_{l}^{i}\right)_{\overrightarrow{j k l}}+\left(R-s R_{s}\right)\left(\delta_{j k} \delta_{l}^{i}\right)_{\overrightarrow{j k l}}\right\} \\
& -\frac{s R_{s s}}{u^{2}}\left\{\left(\delta_{j k} x^{l} y^{i}\right)_{\overrightarrow{j k l}}+\left(\left(x^{k} y^{j}\right)_{\overrightarrow{j k}} \delta_{l}^{i}\right)_{\overrightarrow{j k l}}\right\} \\
& +\frac{1}{u^{3}}\left\{-R+s R_{s}+s^{2} R_{s s}\right\}\left(y^{j} y^{k} \delta_{l}^{i}\right)_{\overrightarrow{j k l}} \\
& +\frac{1}{u^{3}}\left\{-R+s R_{s}+s^{2} R_{s s}\right\} y^{i}\left(\delta_{j k} y^{l}\right)_{\overrightarrow{j k l}} \\
& +\frac{1}{u^{5}}\left\{3 R-3 s R_{s}-6 s^{2} R_{s s}-s^{3} R_{s s s}\right\} y^{i} y^{k} y^{l} y^{i}+\frac{R_{s s s}}{u^{2}} x^{j} x^{k} x^{l} y^{i} \\
& +\frac{s}{u^{4}}\left\{3 R_{s s}+s R_{s s s}\right\}\left(x^{j} y^{k} y^{l}\right)_{\overrightarrow{j k l}} y^{i} \\
& +\frac{1}{u^{3}}\left\{-R_{s s}-s R_{s s s}\right\}\left(x^{j} x^{k} y^{l}\right)_{\overrightarrow{j k l}} y^{i} .
\end{aligned}
$$


Voltando para (2.21), agora calculamos as derivadas envolvendo $Q=Q(r, s)$. Segue de (2.1) a (2.16) que

$$
\begin{aligned}
& \left(u^{2} Q\right)_{j k l}=\left(u_{j k}^{2} Q_{l}\right)_{\overrightarrow{j k l}}+\left(u_{j}^{2} Q_{k l}\right)_{\overrightarrow{j k l}}+u^{2} Q_{j k l} \\
& =2 Q_{s}\left(s_{l} \delta_{j k}\right)_{\overrightarrow{j k l}}+2 Q_{s s}\left(y^{j} s_{k} s_{l}\right)_{\overrightarrow{j k l}}+2 Q_{s}\left(y^{j} s_{k l}\right)_{\overrightarrow{j k l}} \\
& +u^{2}\left[Q_{s s s} s_{j} s_{k} s_{l}+Q_{s s}\left(s_{j k} s_{l}\right)_{\overrightarrow{j k l}}+Q_{s} s_{j k l}\right] \\
& =2 Q_{s}\left(\left(\frac{x^{k}}{u}-\frac{s}{u^{2}} y^{l}\right) \delta_{j k}\right)_{\overrightarrow{j k l}}+\frac{2 Q_{s s}}{u^{2}}\left(\left(x^{k} x^{l}-\frac{s}{u}\left(x^{k} y^{l}+x^{l} y^{k}\right)+\frac{s^{2}}{u^{2}} y^{l} y^{k}\right) y^{j}\right)_{\overrightarrow{j k l}} \\
& +\frac{2 Q_{s}}{u^{2}}\left(\left(\frac{3 s}{u^{2}} y^{k} y^{l}-s \delta_{k l}-\frac{\left(x^{k} x^{l}+x^{l} y^{k}\right)}{u}\right) y^{j}\right)_{\overrightarrow{j k l}} \\
& +\frac{Q_{s s s}}{u}\left[x^{j} x^{k} x^{l}-\frac{s}{u}\left(x^{j} x^{l} y^{k}\right)_{\overrightarrow{j k l}}+\frac{s^{2}}{u^{2}}\left(x^{j} y^{k} y^{l}\right)_{\overrightarrow{j k l}}-\frac{s^{3}}{u^{3}} y^{j} y^{k} y^{l}\right] \\
& +\frac{Q_{s s}}{u}\left(\frac{3 s}{u^{2}} x^{l} y^{j} y^{k}-\frac{3 s^{2}}{u^{3}} y^{j} y^{k} y^{l}-s x^{l} \delta_{j k}+\frac{s^{2}}{u} y^{l} \delta_{j k}-\frac{x^{k} y^{j}+x^{j} y^{k}}{u} x^{l}\right. \\
& \left.+\frac{s}{u^{2}}\left(x^{k} y^{j}+x^{j} y^{k}\right) x^{l}\right)_{\overrightarrow{j k l}} \\
& +\frac{Q_{s}}{u}\left[\left(\left(\frac{3 y^{k} y^{l}}{u^{2}}-\delta_{k l}\right) x^{j}\right)_{\overrightarrow{j k l}}+\frac{3 s}{u}\left(\left(y^{j} \delta_{k l}\right)_{\overrightarrow{j k l}}-\frac{5 y^{j} y^{k} y^{l}}{u^{2}}\right)\right] \\
& =\frac{1}{u}\left[2 Q_{s}\left(\delta_{j k} x^{l}\right)_{\overrightarrow{j k l}}+Q_{s s s} x^{j} x^{k} x^{l}-s Q_{s s}\left(x^{l} \delta_{j k}\right)_{\overrightarrow{j k l}}-Q_{s}\left(x^{j} \delta_{k l}\right)_{\overrightarrow{j k l}}\right] \\
& +\frac{1}{u^{2}}\left[-2 s Q_{s}\left(y^{l} \delta_{j k}\right)_{\overrightarrow{j k l}}+2 Q_{s s}\left(y^{j} x^{k} x^{l}\right)_{\overrightarrow{j k l}}-2 s Q_{s}\left(y^{j} \delta_{k l}\right)_{\overrightarrow{j k l}}-s Q_{s s s}\left(x^{j} x^{k} y^{l}\right)_{\overrightarrow{j k l}}\right. \\
& \left.+s^{2} Q_{s s}\left(y^{j} \delta_{k l}\right)_{\overrightarrow{j k l}}-Q_{s s}\left(\left(x^{k} y^{j}+x^{j} y^{k}\right) x^{l}\right)_{\overrightarrow{j k l}}+3 s Q_{s}\left(y^{j} \delta_{k l}\right)_{\overrightarrow{j k l}}\right] \\
& +\frac{1}{u^{3}}\left[-4 s Q_{s s}\left(x^{j} y^{k} y^{l}\right)_{\overrightarrow{j k l}}-2 Q_{s}\left(\left(x^{k} y^{l}+x^{l} y^{k}\right) y^{j}\right)_{\overrightarrow{j k l}}+s^{2} Q_{s s s}\left(x^{j} y^{k} y^{l}\right)_{\overrightarrow{j k l}}\right. \\
& \left.+3 s Q_{s s}\left(x^{j} y^{k} y^{l}\right)_{\overrightarrow{j k l}}+s Q_{s s}\left(\left(x^{k} y^{j}+x^{j} y^{k}\right) y^{l}\right)_{\overrightarrow{j k l}}+3 Q_{s}\left(x^{j} y^{k} y^{l}\right)_{\overrightarrow{j k l}}\right] \\
& +\frac{1}{u^{4}}\left[2 s^{2} Q_{s s}\left(y^{j} y^{k} y^{l}\right)_{\overrightarrow{j k l}}+6 s Q_{s}\left(y^{j} y^{k} y^{l}\right)_{\overrightarrow{j k l}}\right. \\
& \left.-s^{3} Q_{s s s} y^{j} y^{k} y^{l}-3 s^{2} Q_{s s}\left(y^{j} y^{k} y^{l}\right)_{\overrightarrow{j k l}}-15 s Q_{s} y^{j} y^{k} y^{l}\right] \text {. }
\end{aligned}
$$

Logo,

$$
\begin{aligned}
\left(u^{2} Q\right)_{j k l} x^{i}= & \frac{1}{u}\left[\left(Q_{s}-s Q_{s s}\right) x^{i}\left(x^{l} \delta_{j k}\right)_{\overrightarrow{j k l}}\right. \\
& +\frac{1}{u^{3}}\left(s^{2} Q_{s s s}+s Q_{s s}-Q_{s}\right) x^{i}\left(x^{j} y^{k} y^{l}\right)_{\overrightarrow{j k l}} \\
& +\frac{s}{u^{2}}\left[\left(s Q_{s s}-Q_{s}\right) x^{i}\left(y^{j} \delta_{k l}\right)_{\overrightarrow{j k l}}-Q_{s s s} x^{i}\left(x^{j} x^{l} y^{k}\right)_{\overrightarrow{j k l}}\right] \\
& +\frac{x^{i}}{u^{4}}\left[u^{3} Q_{s s s} x^{j} x^{k} x^{l}+s\left(3 Q_{s}-3 s Q_{s s}-s^{2} Q_{s s s}\right) y^{j} y^{k} y^{l}\right] .
\end{aligned}
$$


Segue de (2.22) e de (2.23) que vale (2.17).

A Proposição 2.1.1 sugere a seguinte Proposição.

Proposição 2.1.2. Seja $F=|y| \phi\left(|x|, \frac{\langle x, y\rangle}{|y|}\right)$ uma métrica de Finsler esfericamente simétrica sobre $M_{s}^{n} \subset \mathbb{R}^{n}$. Então F tem curvatura de Douglas nula se, e somente se,

$$
Q_{s}-s Q_{s s}=0
$$

onde $Q$ e s são dadas por (1.49), isto é, $Q(r, s)$ tem a forma

$$
Q(r, s)=\frac{1}{2} f(r) s^{2}+g(r)
$$

onde $f$ e $g$ são funções diferenciáveis de $r \in I \subset \mathbb{R}$.

Demonstração. Suponha que $F=|y| \phi\left(|x|, \frac{\langle x, y\rangle}{|y|}\right)$ é uma métrica de Douglas, então $D_{j}{ }^{i} k l=0$. Considere $j=k=l=i$, isto nos dá em (1.49)

$$
\frac{3}{u} R_{s s}\left(x^{i}\right)^{2}+\frac{3}{u}\left(R-s R_{s}\right)+\frac{3}{u}\left(Q_{s}-s Q_{s s}\right)\left(x^{i}\right)^{2}+\frac{Q_{s s s}}{u}\left(x^{i}\right)^{4} \equiv 0\left(\bmod y^{i}\right) .
$$

Sendo $R$ e $Q$ homogêneas de grau zero em $y$ e sendo válida a igualdade acima para todo $x \in M_{s}^{n}$ podemos afirmar que,

$$
R-s R_{s}=0
$$

e

$$
R_{s s}+Q_{s}-s Q_{s s}=0, \quad Q_{s s s}=0 .
$$

Derivando (2.26) em relação a $s$

$$
\frac{\partial}{\partial s}\left(R-s R_{s}\right)=R_{s}-s R_{s s}-R_{s}
$$

obtemos

$$
R_{s s}=0
$$

Substituindo (2.28) na primeira identidade de (2.27), temos (2.24). Derivando (2.24) em relação a $s$ temos $Q_{s s s}=0$. Reciprocamente, suponha que $F$ satisfaz (2.24). De (2.24) temos que $Q_{s s s}=0$ e $Q=Q(r, s)$ é dado por (2.25). De (2.18) temos que

$$
R=-\frac{s}{n+1}\left[2 g(r)+r^{2} f(r)\right]:=c(r) s .
$$

Usando (2.25) e (2.29), temos de (2.20) que

$$
\begin{aligned}
G^{i}-\frac{1}{n+1} \sum_{j} \frac{\partial G^{j}}{\partial y^{j}} y^{i} & =u R y^{i}+u^{2} Q x^{i} \\
& =u c(r) s y^{i}+\frac{1}{2}(u s)^{2} f(r) x^{i}+g(r) x^{i}|y|^{2} \\
& =c(r)\langle x, y\rangle y^{i}+\frac{1}{2} f(r)\langle x, y\rangle^{2} x^{i}+g(r) x^{i}|y|^{2} .
\end{aligned}
$$


Por isso podemos ver que

$$
G^{i}-\frac{1}{n+1} \sum_{j} \frac{\partial G^{j}}{\partial y^{j}} y^{i}
$$

é quadrático em $y=\left.y^{j} \frac{\partial}{\partial x^{j}}\right|_{x}$. Usando isto na definição de curvatura de Douglas (equação (1.28)), temos que $F=|y| \phi\left(|x|, \frac{\langle x, y\rangle}{|y|}\right)$ tem curvatura de Douglas nula.

Assim, acabamos de mostrar que (2.24) é condição necessária e suficiente para que a curvatura de Douglas de $F$ seja nula. 


\subsection{Caracterização das Métricas de Douglas Esfericamente Simé- tricas}

Agora podemos enunciar o seguinte teorema de caracterização das métricas de Douglas esfericamente simétricas

Teorema 2.2.1. Uma métrica de Finsler esfericamente simétrica $F(x, y)=|y| \phi(r, s)$ sobre $M_{s}^{n} \in \mathbb{R}^{n}$ é do tipo Douglas se, e somente se, $\phi>0$ satisfaz

$$
\left[\left(r^{2}-s^{2}\right)\left(2 g+f s^{2}\right)-1\right] r \phi_{s s}-s \phi_{r s}+\phi_{r}+r\left(2 g+f s^{2}\right)\left(\phi-s \phi_{s}\right)=0,
$$

para funções $f=f(r)$ e $g=g(r)$ diferenciáveis, onde

$$
r:=|x|, \quad s:=\frac{\langle x, y\rangle}{|y|} .
$$

Demonstração. A prova segue diretamente da Proposição 2.1.2. De fato, da definição de $Q$ que foi dado em (1.49) e a identidade (2.25) temos

$$
\frac{1}{2 r} \frac{r \phi_{s s}-\phi_{r}+s \phi_{r s}}{\phi-s \phi_{s}+\left(r^{2}-s^{2}\right) \phi_{s s}}=g(r)+\frac{1}{2} s^{2} f(r) .
$$

Multiplicando ambos os lados por $2 r\left(\phi-s \phi_{s}+\left(r^{2}-s^{2}\right) \phi_{s s}\right)$,

$$
r \phi_{s s}-\phi_{r}+s \phi_{r s}=r\left(2 g(r)+s^{2} f(r)\right)\left(\phi-s \phi_{s}\right)+r\left(r^{2}-s^{2}\right)\left(2 g(r)+s^{2} f(r)\right) \phi_{s s} .
$$

Como uma consequência imediata deste teorema, considerando $f=g=0$ em (2.30), temos o seguinte resultado obtido por Huang e Mo (ver [15] Teorema 1.1).

Corolário 2.2.1. Seja $F=|y| \phi\left(|x|, \frac{\langle x, y\rangle}{|y|}\right)$ uma métrica de Finsler esfericamente simétrica sobre $\mathbb{B}^{n}(\nu)$. Então $F=F(x, y)$ é projetivamente plana se, e somente se, $\phi=\phi(r, s)$ satisfaz

$$
s \phi_{r s}+r \phi_{s s}-\phi_{r}=0
$$

Embora a equação (2.30) seja de segunda ordem, afortunadamente, é possível integrá-la sob certas hipóteses.

Sejam $f(r)$ e $g(r)$ funções diferenciáveis tais que as seguintes expressões estejam bem definidas.

$$
\int 2 r\left(2 g+r^{2} f\right) d r \quad \text { e } \quad \int 2 r f e^{\int 2 r\left(2 g+r^{2} f\right) d r} d r
$$


Adicionalmente, precisamos definir a seguinte função auxiliar

$$
\varphi(r, s)=\frac{r^{2}-s^{2}}{\left(r^{2}-s^{2}\right) \int 2 r f e^{\int 2 r\left(2 g+r^{2} f\right) d r} d r-e^{\int 2 r\left(2 g+r^{2} f\right) d r}}
$$

Observação 2.2.1. Observe que $r^{2}(x)-s^{2}(x, y)=|x|^{2}\left(1-\cos ^{2} \theta\right) \geq 0$, onde $\theta$ é o ângulo euclidiano entre $x$ e $y$. Por isso, $r^{2}(x)-s^{2}(x, y)>0$, sempre que $x \neq 0$ e $y$ não é múltiplo de $x$. Além de isso, $s \neq 0$ para $y$ não ortogonal a $x$. Como o Teorema 2.2.2 requer $r^{2}-s^{2}>0$ e $s \neq 0$, então o domínio da métrica de Douglas esfericamente simétrica pode ser a região anular. Além disso, devido ao fato de que a função $\eta(\varphi)$ é arbitrária (sempre que tenha sentido (2.33) ), pode-se obter uma função $\phi$, tal que a métrica esteja definida sobre $\mathbb{B}^{n}(\nu) \in \mathbb{R}^{n}$ ou também sobre $\mathbb{R}^{n}$ (ver Corolários 2.3.1, 2.3.2 e 2.3.3).

Teorema 2.2.2. Sejam $f(r)$ e $g(r)$ funções diferenciáveis de $r \in I \subset \mathbb{R}$ tal que as condições em (2.31) são satisfeitas. Então a solução geral de (2.30), quando $r^{2}-s^{2}>0 e$ $s \neq 0$ é dada por

$$
\phi(r, s)=s\left(h(r)-\int \frac{\eta(\varphi(r, s))}{s^{2} \sqrt{r^{2}-s^{2}}} d s\right),
$$

onde $\varphi$ é dada por (2.32), h e $\eta$ são funções diferenciáveis de $r$ e $\varphi$, respectivamente.

Qualquer métrica de Douglas esfericamente simétrica sobre $M \in \mathbb{R}^{n}$ é dada por

$$
F(x, y)=<x, y>\left(h(r)-\int \frac{\eta(\varphi(r, s))}{s^{2} \sqrt{r^{2}-s^{2}}} d s\right),
$$

onde $\varphi$ é da forma (2.32) e $\eta$ satisfaz

$$
\frac{-\sqrt{r^{2}-s^{2}}}{s} \frac{\partial \eta}{\partial s}>0, \text { se } n \geq 2,
$$

com a condição adicional

$$
\frac{\eta}{\sqrt{r^{2}-s^{2}}}>0, \text { se } n \geq 3
$$

\section{Demonstração.}

Como $r^{2}-s^{2}>0$ e $s \neq 0$, a equação (2.30) é equivalente a:

$$
\left[1-\left(r^{2}-s^{2}\right)\left(2 g(r)+f(r) s^{2}\right)\right] r \psi_{s}(r, s)+s \psi_{r}(r, s)=0,
$$

onde

$$
\psi=\left(\phi-s \phi_{s}\right) \sqrt{r^{2}-s^{2}}
$$


De fato, de (2.38) temos

$$
\begin{aligned}
\psi_{r} & =\left(\phi-s \phi_{s}\right)_{r} \sqrt{r^{2}-s^{2}}+\left(\phi-s \phi_{s}\right) \frac{r}{\sqrt{r^{2}-s^{2}}}, \\
\psi_{s} & =\left(\phi-s \phi_{s}\right)_{s} \sqrt{r^{2}-s^{2}}-\left(\phi-s \phi_{s}\right) \frac{s}{\sqrt{r^{2}-s^{2}}} \\
& =-s \phi_{s s} \sqrt{r^{2}-s^{2}}-\left(\phi-s \phi_{s}\right) \frac{s}{\sqrt{r^{2}-s^{2}}},
\end{aligned}
$$

substituindo estas duas últimas igualdades em (2.37) temos,

$$
\begin{aligned}
0= & {\left[1-\left(r^{2}-s^{2}\right)\left(2 g(r)+f(r) s^{2}\right)\right] r\left\{-s \phi_{s s} \sqrt{r^{2}-s^{2}}-\left(\phi-s \phi_{s}\right) \frac{s}{\sqrt{r^{2}-s^{2}}}\right\} } \\
& +s\left\{\left(\phi-s \phi_{s}\right)_{r} \sqrt{r^{2}-s^{2}}+\left(\phi-s \phi_{s}\right) \frac{r}{\sqrt{r^{2}-s^{2}}}\right\} \\
= & {\left[1-\left(r^{2}-s^{2}\right)\left(2 g(r)+f(r) s^{2}\right)\right] r\left\{-s \phi_{s s} \sqrt{r^{2}-s^{2}}\right] } \\
& +\sqrt{r^{2}-s^{2}}\left(2(r) g+f(r) s^{2}\right) r s\left(\phi-s \phi_{s}\right)+s \sqrt{r^{2}-s^{2}}\left(\phi-s \phi_{s}\right)_{r},
\end{aligned}
$$

finalmente fatorando $s \sqrt{r^{2}-s^{2}}$ concluímos que (2.30) e (2.37) são equivalentes. Logo, é suficiente achar a solução geral da equação (2.37).

Assim, do Lema 1.8.1 temos que $\psi(r, s)=\eta(\varphi)$, para alguma função $\eta$ de $\varphi$ definida em (2.32). Podemos concluir então de (2.38) que

$$
\phi-s \phi_{s}=\eta\left(\frac{r^{2}-s^{2}}{\left(r^{2}-s^{2}\right) \int 2 r f e^{\int 2 r\left(2 g+r^{2} f\right) d r} d r-e^{\int 2 r\left(2 g+r^{2} f\right) d r}}\right) / \sqrt{r^{2}-s^{2}} .
$$

Observe que

$$
\phi-s \phi_{s}=-s^{2}\left(\frac{\phi}{s}\right)_{s}
$$

portanto, (2.33) é a solução geral de (2.30).

Agora, verificaremos as condições (1.65) e (1.66), para que $F$ seja uma métrica de Finsler. Derivando $\varphi$, definido em (2.32), em relação a $s$ temos: 


$$
\begin{aligned}
& \varphi_{s}=\frac{-2 s\left(\left(r^{2}-s^{2}\right) \int 2 r f e^{\int 2 r\left(2 g+r^{2} f\right) d r} d r-e^{\int 2 r\left(2 g+r^{2} f\right) d r}\right)+\left(r^{2}-s^{2}\right)\left(2 s \int 2 r f e^{\int 2 r\left(2 g+r^{2} f\right) d r} d r\right)}{\left(\left(r^{2}-s^{2}\right) \int 2 r f e^{\int 2 r\left(2 g+r^{2} f\right) d r} d r-e^{\int 2 r\left(2 g+r^{2} f\right) d r}\right)^{2}} \\
& =\frac{2 s e^{\int 2 r\left(2 g+r^{2} f\right) d r}}{\left(\left(r^{2}-s^{2}\right) \int 2 r f e^{\int 2 r\left(2 g+r^{2} f\right) d r} d r-e^{\int 2 r\left(2 g+r^{2} f\right) d r}\right)^{2}} \\
& =\frac{2 s e^{\int 2 r\left(2 g+r^{2} f\right) d r}}{\left(r^{2}-s^{2}\right)^{2}} \varphi^{2}
\end{aligned}
$$

Agora, derivando a igualdade (2.39) em relação a $s$ temos:

$$
\begin{aligned}
\left(\phi-s \phi_{s}\right)_{s} & =\left(\frac{\eta}{\sqrt{r^{2}-s^{2}}}\right)_{s} \\
-s \phi_{s s} & =\frac{1}{\sqrt{r^{2}-s^{2}}} \eta_{s}+\frac{s}{\left(r^{2}-s^{2}\right)^{3 / 2}} \eta
\end{aligned}
$$

ou seja,

$$
\left(r^{2}-s^{2}\right) \phi_{s s}=-\frac{\sqrt{r^{2}-s^{2}}}{s} \eta_{s}-\frac{1}{\sqrt{r^{2}-s^{2}}} \eta
$$

Assim, de (2.32) a expressão (1.65) para $F=|y| \phi\left(|x|, \frac{\langle x, y>}{|y|}\right)$ é dada por,

$$
-\frac{\sqrt{r^{2}-s^{2}}}{s} \eta_{s}>0
$$

ou equivalentemente, usando a regra da cadeia e (2.40)

$$
-\frac{2 e^{\int 2 r\left(2 g+r^{2} f\right) d r}}{\left(r^{2}-s^{2}\right)^{3 / 2}} \varphi^{2} \eta^{\prime}>0,
$$

A condição (2.36) é obtida diretamente de (2.39).

Portanto, quando $\phi$ é dada por (2.33), $F$ dado por (2.34) define uma métrica de Finsler se, e somente se, as desigualdades (2.35) e (2.36) são satisfeitas. 


\subsection{Exemplos de Famílias de Métricas de Douglas}

Nesta seção, obtemos novas famílias de métricas de Douglas como corolários do Teorema 2.2.2.

Considerando no Teorema 2.2.2 $f=0$ e $\eta(\varphi)=\sqrt{r^{2}-s^{2}} e^{-\int 2 r g(r) d r}$, obtemos o seguinte.

Corolário 2.3.1. Seja $g(r)$ uma função diferenciável tal que $\int 2 r g(r) d r$ está bem definido, e seja $\phi(r, s)$ uma função positiva dada por

$$
\phi(r, s)=s h(r)+A e^{-\int 2 r g(r) d r},
$$

onde $h(r)$ é uma função diferenciável arbitrária. Então a métrica de Finsler

$$
F(x, y)=|y| \phi\left(|x|, \frac{<x, y>}{|y|}\right)
$$

é uma métrica de Douglas esfericamente simétrica definida sobre $\mathbb{B}^{n}(\nu)$.

Em particular, por exemplo, se escolhermos $g(r)=r^{2} / 2$, no Corolário 2.3.1, temos que $\phi=s h(r)+A e^{-r^{4} / 4}$, onde $A>0$. Por isso, para qualquer função diferenciável $h$ de $|x|$ tal que $\phi>0$, temos a seguinte métrica de Douglas,

$$
F(x, y)=\langle x, y\rangle h(|x|)+A|y| e^{-\frac{|x|^{4}}{4}} .
$$

Outra família de métricas de Douglas é obtida do Teorema 2.2.2, considerando $f(r)=g(r)=0, \operatorname{assim} \varphi=-\left(r^{2}-s^{2}\right) \mathrm{e}$

$$
\phi(r, s)=s h(r)+\frac{\eta(\varphi(r, s))}{\sqrt{r^{2}-s^{2}}}-s \int \frac{1}{s} \frac{\partial}{\partial s}\left(\frac{\eta(\varphi(r, s))}{\sqrt{r^{2}-s^{2}}}\right) d s .
$$

Escolhendo $\eta(\varphi)=\sqrt{-\varphi}\left(\frac{-(\mu+1) \varphi+1}{(-\mu \varphi+1)^{\frac{3}{2}}}\right)$, para uma constante $\mu \in \mathbb{R}$, temos:

Corolário 2.3.2. Seja $\phi(r, s)$ uma função definida por

$$
\phi(r, s)=\operatorname{sh}(r)+\frac{\left[1+(1+\mu) r^{2}\right]\left[1+\mu\left(r^{2}-s^{2}\right)\right]+s^{2}}{\sqrt{\left(1+\mu\left(r^{2}-s^{2}\right)\right)}\left(1+\mu r^{2}\right)^{2}},
$$

onde $\mu \in \mathbb{R}$, e $h(r)$ é qualquer função tal que $\phi(r, s)$ seja positiva. Então a seguinte métrica de Finsler sobre $\mathbb{B}^{n}(\nu) \in \mathbb{R}^{n}$, onde $\nu=1 / \sqrt{-\mu}$ se $\mu<0$

$$
F(x, y):=|y| \phi\left(|x|, \frac{<x, y>}{|y|}\right)
$$

é uma métrica de Douglas esfericamente simétrica. Ademais, F é projetivamente plana. 
Em particular, escolhendo no Corolário 2.3.2

$$
h(r)=\frac{2 \sqrt{1+(\mu+1) r^{2}}}{\left(1+\mu r^{2}\right)^{2}}
$$

obtemos o Exemplo 4.3. dado em [40], isto é,

$$
F(x, y)=\frac{\left(\sqrt{1+(1+\mu)|x|^{2}} \sqrt{\left(1+\mu|x|^{2}\right)|y|^{2}-\mu<x, y>^{2}}+<x, y>\right)^{2}}{\left(1+\mu|x|^{2}\right)^{2} \sqrt{\left(1+\mu|x|^{2}\right)|y|^{2}-\mu<x, y>^{2}}} .
$$

Como uma consequência do Teorema 2.2.2, para $f(r)=0$ e $2 g(r)=\frac{\zeta \varepsilon+\kappa^{2}}{\left(\zeta \varepsilon+\kappa^{2}\right) r^{2}+\varepsilon}$, onde $\zeta, \varepsilon, \kappa$ são quaisquer constantes reais tais que $\left(\zeta \varepsilon+\kappa^{2}\right) r^{2}+\varepsilon>0$, temos, $\varphi=$ $\frac{-\left(r^{2}-s^{2}\right)}{\left(\zeta \varepsilon+\kappa^{2}\right) r^{2}+\varepsilon}$, escolhendo $\eta(\varphi)=\varepsilon \sqrt{-\left(\frac{1}{\varphi}+\kappa^{2}\right)^{-1}}$, temos o seguinte Corolário

Corolário 2.3.3. Seja $\phi(r, s)$ uma função definida por

$$
\phi(r, s)=\operatorname{sh}(r)+\frac{\sqrt{\zeta \varepsilon r^{2}+\kappa^{2} s^{2}+\varepsilon}}{\zeta r^{2}+1},
$$

onde $h(r)$ é qualquer função tal que $\phi(r, s)$ é positiva. Para $\nu=1 / \sqrt{-\zeta}$ se $\zeta<0$, temos que a seguinte métrica de Finsler esfericamente simétrica sobre $\mathbb{B}^{n}(\nu) \in \mathbb{R}^{n}$,

$$
F(x, y)=|y| \phi\left(|x|, \frac{<x, y>}{|y|}\right)
$$

é do tipo Douglas.

Em particular, quando

$$
h(r)=\frac{\kappa}{1+\zeta r^{2}}
$$

temos,

$$
F(x, y)=\frac{\sqrt{\kappa^{2}<x, y>^{2}+\varepsilon|y|^{2}\left(1+\zeta|x|^{2}\right)}}{1+\zeta|x|^{2}}+\frac{\kappa<x, y>}{1+\zeta|x|^{2}} .
$$

Quando $\kappa= \pm 1, \zeta=-1$ e $\epsilon=1, F(x, y)$ é a métrica de Funk [7]. Ver Teorema 1.1 em [22] para algumas propriedades desta métrica. 
O seguinte Corolário generaliza um resultado conhecido no caso das métricas esfericamente simétricas projetivamente planas (ver [15]). Primeiramente introduzimos a função erro, também chamada função erro de Gauss, que foi criada para poder calcular a integral da distribuição normal.

A função erro é definida pela integral:

$$
\operatorname{erf}(x)=\frac{2}{\sqrt{\pi}} \int_{0}^{x} e^{-t^{2}} \mathrm{~d} t .
$$

Esta integral não pode ser expressa por funções elementares.

Corolário 2.3.4. Seja $\phi(r, s)$ uma função definida por

$$
\phi(r, s)=s h(r)+\frac{e^{\lambda r^{2-4 \epsilon}}}{r^{2 \epsilon}}\left[e^{-\frac{\lambda}{r^{4 \epsilon}} s^{2}}+\frac{\sqrt{\lambda \pi}}{r^{2 \epsilon}} s \operatorname{erf}\left(\frac{\sqrt{\lambda}}{r^{2 \epsilon}} s\right)\right]
$$

onde $\lambda$ é uma constante positiva, $\epsilon$ é uma constante, erf(.) denota a função erro e h é uma função qualquer de r. Então sobre $M_{s}^{n}$ a seguinte função

$$
F=|y| \phi\left(|r|, \frac{<x, y>}{|y|}\right)
$$

é uma métrica de Finsler do tipo Douglas. Além disso, F é projetivamente plana se, e somente se, $\epsilon=0$.

Demonstração. No Teorema 2.2 .2 considere $f(r)=0$ e $g(r)=\frac{\epsilon}{r^{2}}$ para alguma constante $\epsilon$. É fácil ver que

$$
\int r g(r) d r=\ln r^{\epsilon}, \quad r\left[1-2 r^{2} g(r)\right]=(1-2 \epsilon) r .
$$

Assim,

$$
\phi(r, s)=s h(r)-\frac{s}{r^{2 \epsilon}} \int \frac{\eta\left(r^{-4 \epsilon}\left(r^{2}-s^{2}\right)\right)}{s^{2}} d s
$$

Seja

$$
\eta(u)=e^{\lambda u}
$$

onde $\lambda$ é uma constante positiva, então, temos que

$$
\begin{aligned}
\int \frac{\eta\left(r^{-4 \epsilon}\left(r^{2}-s^{2}\right)\right)}{s^{2}} \mathrm{~d} s & =\int s^{-2} e^{\lambda r^{-4 \epsilon}\left(r^{2}-s^{2}\right)} \mathrm{d} s \\
& =e^{\lambda r^{2-4 \epsilon}} \int s^{-2} e^{\lambda r^{-4 \epsilon} s^{2}} \mathrm{~d} s \\
& =-e^{\lambda r^{2-4 \epsilon}}\left(s^{-1} e^{-\lambda r^{-4 \epsilon} s^{2}}+2 \lambda r^{-4 \epsilon} \int e^{-\lambda r^{-4 \epsilon} s^{2}} \mathrm{~d} s\right) .
\end{aligned}
$$

Reescrevendo (2.44) em termos da função erro e observando que

$$
\int_{0}^{s} e^{-\lambda r^{-4 \epsilon} t^{2}} \mathrm{~d} t=\frac{r^{2 \epsilon} \sqrt{\pi}}{2 \sqrt{\lambda}} \operatorname{erf}\left(\frac{\sqrt{\lambda}}{r^{2 \epsilon}} r\right)
$$


obtemos (2.42)

Na seguinte aplicação do Teorema 2.2.2, obteremos uma família de métricas de Randers, e não precisamos que $f$ seja zero, em contraste com os resultados prévios. Introduziremos as seguintes notações

$$
\begin{gathered}
I(r)=e^{\int 2 r\left(2 g(r)+r^{2} f(r)\right) d r} \\
L(r, s)=I(r)-\left(r^{2}-s^{2}\right) \int 2 r f(r) I(r) d r \\
T(r)=I(r)-r^{2} \int 2 r f(r) I(r) d r .
\end{gathered}
$$

Corolário 2.3.5. Seja $\phi(r, s)$ uma função definida por

$$
\phi(r, s)=\operatorname{sh}(r)+K \frac{\sqrt{L(r, s)}}{T(r)}
$$

onde $L(r, s)$ e $T(r)$ estão definidos em (2.46) e (2.47), $K \in \mathbb{R}^{+}$e $h(r)$ é qualquer função tal que $\phi(r, s)$ seja positiva. Então, para $r$ e s dados por (1.49), a seguinte métrica de Finsler esfericamente simétrica definida sobre $\mathbb{B}^{n}(\nu) \in \mathbb{R}^{n}$,

$$
F(x, y):=|y| \phi\left(|x|, \frac{<x, y>}{|y|}\right),
$$

é do tipo Douglas.

Demonstração. Observe que de (2.46) a expressão (2.32) pode ser reescrita como

$$
\varphi(r, s)=\frac{r^{2}-s^{2}}{-L(r, s)} .
$$

Além disso, observe que

$$
\begin{aligned}
\frac{\partial}{\partial s}\left(\frac{\sqrt{L(r, s)}}{s T(r)}\right) & =\frac{1}{2 s T(r) \sqrt{L(r, s)}} L_{s}(r, s)-\frac{\sqrt{L(r, s)}}{s^{2} T(r)} \\
& =\frac{s \int 2 r f(r) I(r) d r}{s T(r) \sqrt{L(r, s)}}-\frac{I(r)-\left(r^{2}-s^{2}\right) \int 2 r f(r) I(r) d r}{s^{2} T(r) \sqrt{L(r, s)}} \\
& =-\frac{1}{s^{2} \sqrt{L(r, s)}}
\end{aligned}
$$


Escolhendo $\eta(\varphi)=A \sqrt{-\varphi}$, onde $A>0$ é uma constante qualquer e tendo em vista, para $a, b \in \mathbb{R}$, que

$$
\int \frac{1}{\left(a^{2}+b^{2} t^{2}\right)^{\frac{3}{2}}} d t=\frac{t}{a^{2} \sqrt{a^{2}+b^{2} t^{2}}}
$$

segue de (2.33) que

$$
\begin{aligned}
\phi(r, s) & =\operatorname{sh}(r)+\frac{A}{\sqrt{L(r, s)}}+\frac{A s^{2} \int 2 r f(r) I(r) d r}{\left(I(r)-r^{2} \int 2 r f(r) I(r) d r\right) \sqrt{L(r, s)}} \\
& =\operatorname{sh}(r)+\frac{A}{\sqrt{L(r, s)}} \frac{L(r, s)}{T(r)} \\
& =\operatorname{sh}(r)+A \frac{\sqrt{L(r, s)}}{T(r)} .
\end{aligned}
$$

Como

$$
\frac{-\sqrt{r^{2}-s^{2}}}{s} \frac{\partial \eta}{\partial s}=\frac{K I(r)}{(L(r, s))^{\frac{3}{2}}}>0 \quad \text { e } \quad \frac{\eta}{\sqrt{r^{2}-s^{2}}}=\frac{K}{(L(r, s))^{\frac{1}{2}}}>0,
$$

concluímos a prova deste Corolário como consequência do Teorema 2.2.2.

Em particular, quando escolhemos $f=c$ uma constante diferente de zero e $2 g(r)=\left(\nu^{2}-r^{2}\right) c$, onde $\nu$ é uma constante positiva, temos

$$
I(r)=e^{\nu^{2} c r^{2}}, \quad L(r, s)=I(r)\left(1-\frac{r^{2}-s^{2}}{\nu^{2}}\right), \quad T(r)=I(r)\left(1-\frac{r^{2}}{\nu^{2}}\right) .
$$

e por isso,

$$
\phi(r, s)=\operatorname{sh}(r)+\frac{K \nu \sqrt{\nu^{2}-\left(r^{2}-s^{2}\right)}}{e^{\frac{\nu^{2} c r^{2}}{2}}\left(\nu^{2}-r^{2}\right)},
$$

onde $h(r)$ é tal que $\phi$ é positiva. Assim, consegue-se mostrar que

$$
F(x, y)=<x, y>h(|x|)+\frac{K \nu \sqrt{\left(\nu^{2}-|x|^{2}\right)|y|^{2}+<x, y>^{2}}}{e^{\frac{\nu^{2} c|x|^{2}}{2}}\left(\nu^{2}-|x|^{2}\right)}
$$

é uma métrica de Douglas.

Os resultados deste capítulo geraram o artigo [23] publicado em Differential Geometry and its Applications (2013).

Nos capítulos a seguir, daremos mais exemplos de métricas de Douglas esfericamente simétricas (ver Exemplos 4.2.3 e 4.2.4). 


\section{MÉTRICAS DE BERWALD E MÉTRICAS DE LANDSBERG}

\subsection{Métricas de Berwald Esfericamente Simétricas}

Neste capítulo caracterizaremos as métricas de Berwald e as métricas de Landsberg esfericamente simétricas definidas sobre $M_{s}^{n} \subset \mathbb{R}^{n}$.

Proposição 3.1.1. Seja $F=|y| \phi(r, s)$ uma métrica de Finsler esfericamente simétrica sobre $M_{s}^{n} \subset \mathbb{R}^{n}$, então ela é uma métrica de Berwald se, e somente se, para todo $(x, y) \in$ $T M_{s}^{n} \subset \mathbb{R}^{n}$.

$$
\begin{aligned}
0= & \frac{1}{u}\left\{P_{s s}\left(x^{j} x^{k} \delta_{l}^{i}\right)_{\overrightarrow{j k l}}+\left(P-s P_{s}\right)\left(\delta_{j k} \delta_{l}^{i}\right)_{\overrightarrow{j k l}}\right\} \\
& -\frac{s P_{s s}}{u^{2}}\left\{\left(\delta_{j k} x^{l} y^{i}\right)_{\overrightarrow{j k l}}+\left(\left(x^{k} y^{j}\right)_{\overrightarrow{j k}} \delta_{l}^{i}\right)_{\overrightarrow{j k l}}\right\} \\
& +\frac{1}{u^{3}}\left\{-P+s P_{s}+s^{2} P_{s s}\right\}\left(y^{j} y^{k} \delta_{l}^{i}\right)_{\overrightarrow{j k l}} \\
& +\frac{1}{u^{3}}\left\{-P+s P_{s}+s^{2} P_{s s}\right\} y^{i}\left(\delta_{j k} y^{l}\right)_{\overrightarrow{j k l}} \\
& +\frac{1}{u^{5}}\left\{3 P-3 s P_{s}-6 s^{2} P_{s s}-s^{3} P_{s s s}\right\} y^{i} y^{k} y^{l} y^{i}+\frac{P_{s s s}}{u^{2}} x^{j} x^{k} x^{l} y^{i} \\
& +\frac{s}{u^{4}}\left\{3 P_{s s}+s P_{s s s}\right\}\left(x^{j} y^{k} y^{l}\right)_{\overrightarrow{j k l}} y^{i} \\
& +\frac{1}{u^{3}}\left\{-P_{s s}-s P_{s s s}\right\}\left(x^{j} x^{k} y^{l}\right)_{\overrightarrow{j k l}} y^{i} \\
& +\frac{1}{u}\left(Q_{s}-s Q_{s s}\right) x^{i}\left(\delta_{j k} x^{l}\right)_{\overrightarrow{j k l}} \\
& +\frac{1}{u^{3}}\left(s^{2} Q_{s s s}+s Q_{s s}-Q_{s}\right)^{i}\left(x^{j} y^{k} y^{l}\right)_{\overrightarrow{j k l}} \\
& -\frac{s}{u^{2}} Q_{s s s} x^{i}\left(x^{j} x^{l} y^{k}\right)_{\overrightarrow{j k l}}+\frac{1}{u} Q_{s s s} x^{i} x^{j} x^{k} x^{l} \\
& +\frac{1}{u^{2}}\left(s^{2} Q_{s s}-s Q_{s}\right) x^{i}\left(\delta_{j k} y^{l}\right)_{\overrightarrow{j k l}} \\
& +\frac{1}{u^{4}}\left(3 s Q_{s}-3 s^{2} Q_{s s}-s^{3} Q_{s s s}\right) x^{i} y^{j} y^{k} y^{l},
\end{aligned}
$$

onde $Q=Q(r, s)$ e $P=P(r, s)$ foram definidas em (1.49) e (1.50) respectivamente.

Demonstração. Usando a caracterização (1.21) para métricas de Berwald

$$
\frac{\partial^{3} G^{i}}{\partial y^{j} \partial y^{k} \partial y^{l}}=0
$$

onde $G^{i}$ são os coeficientes geodésicos de $F$ dado por (1.48), a prova torna-se análoga à prova da Proposição 2.1.1. (observe (2.20) e (1.48)). 
A seguinte Proposição também é análoga à Proposição 2.1.2.

Proposição 3.1.2. Seja $F=|y| \phi(r, s)$ uma métrica de Finsler esfericamente simétrica sobre $M_{s}^{n} \subset \mathbb{R}^{n}$, então $F$ é de Berwald se, e somente se, existem funções diferenciáveis de $r, c_{1}:=c_{1}(r), f:=f(r)$ e $g:=g(r)$ tal que:

$$
P=c_{1} s, \quad Q=\frac{1}{2} f s^{2}+g .
$$

onde $Q(r, s)$ e $P=P(r, s)$ foram definidas em (1.49) e (1.50).

Demonstração. Da Observação 1.5.1 temos que toda métrica de Berwald é de Douglas, portanto da Proposição 2.1.2 temos que $Q=Q(r, s)$ é da forma (2.25), logo em (3.1) os termos envolvendo $Q$ se anulam.

Considerando $i=j=k=l$, na equação (3.1) temos

$$
3 P_{s s}\left(x^{i}\right)^{2}+\left(P-s P_{s}\right)=0 \quad\left(\bmod \quad y^{i}\right)
$$

$\operatorname{Logo} P_{s s}=0$ e $P-s P_{s}=0$. De $P_{s s}=0$ temos

$$
P=c_{1}(r) s+c_{2}(r)
$$

substituindo em $P-s P_{s}=0$ temos $c_{2}(r)=0$.

Portanto, $P=c_{1}(r) s$.

Reciprocamente, se $P=c_{1}(r) s$ e $Q=\frac{1}{2} f(r) s^{2}+g(r)$ então substituindo em (3.1) obtemos uma identidade.

Teorema 3.1.3. Uma métrica de Finsler esfericamente simétrica $F(x, y)=|y| \phi(r, s)$ sobre $M_{s}^{m} \subset \mathbb{R}^{n}$ é do tipo Berwald se, e somente se, $\phi$ satisfaz o seguinte sistema de equações diferenciais parciais:

$$
\begin{gathered}
{\left[\left(r^{2}-s^{2}\right)\left(2 g+f s^{2}\right)-1\right] r \phi_{s s}-s \phi_{r s}+\phi_{r}+r\left(2 g+f s^{2}\right)\left(\phi-s \phi_{s}\right)=0} \\
{\left[1-\left(r^{2}-s^{2}\right)\left(2 g+f s^{2}\right)\right] r \phi_{s}+s \phi_{r}-s r\left(2 g+s^{2} f\right) \phi-2 r s c_{1} \phi=0}
\end{gathered}
$$

para funções $f=f(r), g=g(r)$ e $c_{1}=c_{1}(r)$, onde $r$ e s estão definidos em (1.49).

Demonstração. A prova segue das definições de $Q$ e $P$ dadas por (1.49) e (1.50) respectivamente, substituindo estas equações em (3.3). 


\subsection{Métricas de Landsberg Esfericamente Simétricas}

Nesta seção provaremos que toda métrica de Finsler esfericamente simétrica do tipo Landsberg definida sobre $M_{s}^{n} \subset \mathbb{R}^{n}$ é do tipo Berwald. As demonstrações serão apresentadas brevemente pois estão baseadas no trabalho de X. Mo e L. Zhou [24] que provaram que toda métrica de Finsler esfericamente simétrica do tipo Landsberg definida sobre $\mathbb{R}^{n}$ é Riemanniana.

Proposição 3.2.1. Seja $F=|y| \phi(r, s)$ uma métrica de Finsler esfericamente simétrica sobre $M_{s}^{n} \subset \mathbb{R}^{n}$, então ela é uma métrica de Landsberg se, e somente se,

$$
\begin{aligned}
0= & L_{1} x^{j} x^{k} x^{l}+L_{2}\left(x^{j} \delta_{k l}\right)_{\overrightarrow{j k l}}+\frac{1}{u^{2}} L_{3}\left(x^{j} x^{k} y^{l}\right)_{\overrightarrow{j k l}}+\frac{1}{u} L_{4}\left(x^{j} y^{l} y^{l}\right)_{\overrightarrow{j k l}}+ \\
& +\frac{1}{u} L_{5}\left(\delta_{j k} y^{l}\right)_{\overrightarrow{j k l}}+\frac{1}{u^{3}} L_{6} y^{j} y^{k} y^{l},
\end{aligned}
$$

onde $r=|x|, s=\frac{<x, y>}{|y|} e$

$$
\begin{aligned}
& L_{1}:=3 \phi_{s} P_{s s}+\phi P_{s s s}+\left(s \phi+\left(r^{2}-s^{2}\right) \phi_{s}\right) Q_{s s s}, \\
& L_{2}:=-s \phi P_{s s}+\phi_{s}\left(P-s P_{s}\right)+\left(s \phi+\left(r^{2}-s^{2}\right) \phi_{s}\right)\left(Q_{s}-s Q_{s s}\right), \\
& L_{3}:=-s L_{1}, \\
& L_{4}:=s^{2} L_{1}-L_{2}, \\
& L_{5}:=-s L_{2}, \\
& L_{6}:=-s^{3} L_{1}+3 s L_{2} .
\end{aligned}
$$

Demonstração. Da Definição 1.1 procedemos a calcular $L_{i j k}$ dado por (1.26).

A primeira parte da prova (que é calcular os $B_{j k l}^{i}$ ), é igual a prova da Proposição 2.1.1, isto é,

$$
\begin{aligned}
B_{j k l}^{i}= & \frac{1}{u} P_{s s}\left(\delta_{j}^{i} x^{k} x^{l}\right)_{\overrightarrow{j k l}}+\frac{1}{u}\left(P-s P_{s}\right)\left(\delta_{j}^{i} \delta_{k l}\right)_{\overrightarrow{j k l}} \\
& -\frac{s}{u^{2}} P_{s s}\left(\delta_{j}^{i}\left(x^{k} y^{l}+x^{l} y^{k}\right)\right)_{\overrightarrow{j k l}} \\
& -\frac{s}{u^{2}} P_{s s} y^{i}\left(\delta_{j k} x^{l}\right)_{\overrightarrow{j k l}}+\frac{1}{u}\left(Q_{s}-s Q_{s s}\right) x^{i}\left(\delta_{j k} x^{l}\right)_{\overrightarrow{j k l}} \\
& +\frac{1}{u^{3}}\left(s^{2} P_{s s}+s P_{s}-P\right)\left(\delta_{j}^{i} y^{k} y^{l}\right)_{\overrightarrow{j k l}} \\
& +\frac{1}{u^{3}}\left(s^{2} P_{s s}+s P_{s}-P\right) y^{i}\left(\delta_{j k} y^{l}\right)_{\overrightarrow{j k l}} \\
& +\frac{1}{u^{5}}\left(3 P-s^{3} P_{s s s}-6 s^{2} P_{s s}-3 s P_{s}\right) y^{i} y^{j} y^{k} y^{l} \\
& +\frac{1}{u^{4}}\left(s^{2} P_{s s s}+3 s P_{s s}\right) y^{i}\left(y^{j} y^{k} x^{l}\right)_{\overrightarrow{j k l}}+\frac{1}{u^{2}} P_{s s s} y^{i} x^{j} x^{k} x^{l}
\end{aligned}
$$




$$
\begin{aligned}
& -\frac{1}{u^{3}}\left(P_{s s}+s P_{s s s}\right) y^{i}\left(y^{j} x^{k} x^{l}\right)_{\overrightarrow{j k l}} \\
& +\frac{1}{u^{3}}\left(s^{2} Q_{s s s}+s Q_{s s}-Q_{s}\right) x^{i}\left(x^{j} y^{k} y^{l}\right)_{\overrightarrow{j k l}} \\
& -\frac{s}{u^{2}} Q_{s s s} x^{i}\left(x^{j} x^{l} y^{k}\right)_{\overrightarrow{j k l}}+\frac{x^{i}}{u} Q_{s s s} x^{j} x^{k} x^{l} \\
& +\frac{1}{u^{2}}\left(s^{2} Q_{s s}-s Q_{s}\right) x^{i}\left(\delta_{j k} y^{l}\right)_{\overrightarrow{j k l}} \\
& +\frac{1}{u^{4}}\left(3 s Q_{s}-3 s^{2} Q_{s s}-s^{3} Q_{s s s}\right) x^{i} y^{j} y^{k} y^{l} .
\end{aligned}
$$

De (1.51) procedemos a calcular $F_{y^{i}} B_{j k l}^{i}$, para isso primeiro calculamos $y^{i} B_{j k l}^{i}$,

$$
\begin{aligned}
y^{i} B_{j k l}^{i}= & \frac{P_{s s}}{u}\left(y^{j} x^{k} x^{l}\right)_{\overrightarrow{j k l}}+\frac{1}{u}\left(P-s P_{s}\right)\left(y^{j} \delta_{k l}\right)_{\overrightarrow{j k l}} \\
& -\frac{s}{u^{2}} P_{s s}\left(2 y^{j} x^{k} y^{l}\right)_{\overrightarrow{j k l}} \\
& -\frac{s}{u^{2}} P_{s s} u^{2}\left(\delta_{j k} x^{l}\right)_{\overrightarrow{j k l}}+\frac{1}{u}\left(Q_{s}-s Q_{s s}\right) u s\left(\delta_{j k} x^{l}\right)_{\overrightarrow{j k l}} \\
& +\frac{1}{u^{3}}\left(s^{2} P_{s s}+s P_{s}-P\right)\left(y^{j} y^{k} y^{l}\right)_{\overrightarrow{j k l}} \\
& +\frac{1}{u^{3}}\left(s^{2} P_{s s}+s P_{s}-P\right) u^{2}\left(\delta_{j k} y^{l}\right)_{\overrightarrow{j k l}} \\
& +\frac{1}{u^{5}}\left(3 P-s^{3} P_{s s s}-6 s^{2} P_{s s}-3 s P_{s}\right) u^{2} y^{j} y^{k} y^{l} \\
& +\frac{1}{u^{4}}\left(s^{2} P_{s s s}+3 s P_{s s}\right) u^{2}\left(y^{j} y^{k} x^{l}\right)_{\overrightarrow{j k l}}+\frac{1}{u^{2}} P_{s s s} u^{2} x^{j} x^{k} x^{l} \\
& -\frac{1}{u^{3}}\left(P_{s s}+s P_{s s s}\right) u^{2}\left(y^{j} x^{k} x^{l}\right)_{\overrightarrow{j k l}} \\
& +\frac{1}{u^{3}}\left(s^{2} Q_{s s s}+s Q_{s s}-Q_{s}\right) u s\left(x^{j} y^{k} y^{l}\right)_{\overrightarrow{j k l}} \\
& -\frac{s}{u^{2}} Q_{s s s} u s\left(x^{j} x^{l} y^{k}\right)_{\overrightarrow{j k l}}+s Q_{s s s} x^{j} x^{k} x^{l} \\
& +\frac{1}{u^{2}}\left(s^{2} Q_{s s}-s Q_{s}\right) u s\left(\delta_{j k} y^{l}\right)_{\overrightarrow{j k l}} \\
& +\frac{1}{u^{4}}\left(3 s Q_{s}-3 s^{2} Q_{s s}-s^{3} Q_{s s s}\right) u s y^{j} y^{k} y^{l} .
\end{aligned}
$$

Reagrupando a expressão anterior, temos

$$
\begin{aligned}
y^{i} B_{j k l}^{i}= & \frac{1}{u}\left(P_{s s}-P_{s s}-s P_{s s s}-s^{2} Q_{s s s}\right)\left(y^{j} x^{k} x^{l}\right)_{\overrightarrow{j k l}} \\
& +\frac{1}{u}\left(P-s P_{s}+s^{2} P_{s s}+s P_{s}-P+s^{3} Q_{s s}-s^{2} Q_{s}\right)\left(y^{j} \delta_{k l}\right)_{\overrightarrow{j k l}} \\
& +\frac{1}{u^{2}}\left(-2 s P_{s s}+s^{2} P_{s s s}+3 s P_{s s}+s^{3} Q_{s s s}+s^{2} Q_{s s}-s Q_{s}\right)\left(y^{j} x^{k} y^{l}\right)_{\overrightarrow{j k l}} \\
& +\left(-s P_{s s}+s Q_{s}-s^{2} Q_{s s}\right)\left(\delta_{j k} x^{l}\right)_{\overrightarrow{j k l}} \\
& +\frac{1}{u^{3}}\left(3 s^{2} P_{s s}+3 s P_{s}-s^{3} P_{s s s}-6 s^{2} P_{s s}-3 s P_{s}+3 s^{2} Q_{s}-3 s^{3} Q_{s s}-s^{4} Q_{s s s}\right) y^{j} y^{k} y^{l} \\
& +\left(P_{s s s}+s Q_{s s s}\right) x^{j} x^{k} x^{l} .
\end{aligned}
$$


Por outro lado, podemos calcular $x^{i} B_{j k l}^{i}$,

$$
\begin{aligned}
x^{i} B_{j k l}^{i}= & \frac{P_{s s}}{u}\left(x^{j} x^{k} x^{l}\right)_{\overrightarrow{j k l}}+\frac{1}{u}\left(P-s P_{s}\right)\left(x^{j} \delta_{k l}\right)_{\overrightarrow{j k l}} \\
& -\frac{s}{u^{2}} P_{s s}\left(2 x^{j} x^{k} y^{l}\right)_{\overrightarrow{j k l}} \\
& -\frac{s}{u^{2}} P_{s s} u s\left(\delta_{j k} x^{l}\right)_{\overrightarrow{j k l}}+\frac{1}{u}\left(Q_{s}-s Q_{s s}\right) r^{2}\left(\delta_{j k} x^{l}\right)_{\overrightarrow{j k l}} \\
& +\frac{1}{u^{3}}\left(s^{2} P_{s s}+s P_{s}-P\right)\left(x^{j} y^{k} y^{l}\right)_{\overrightarrow{j k l}} \\
& +\frac{1}{u^{3}}\left(s^{2} P_{s s}+s P_{s}-P\right) u s\left(\delta_{j k} y^{l}\right)_{\overrightarrow{j k l}} \\
& +\frac{1}{u^{5}}\left(3 P-s^{3} P_{s s s}-6 s^{2} P_{s s}-3 s P_{s}\right) u s y^{j} y^{k} y^{l} \\
& +\frac{1}{u^{4}}\left(s^{2} P_{s s s}+3 s P_{s s}\right) u s\left(y^{j} y^{k} x^{l}\right)_{\overrightarrow{j k l}}+\frac{1}{u^{2}} P_{s s s} u s x^{j} x^{k} x^{l} \\
& -\frac{1}{u^{3}}\left(P_{s s}+s P_{s s s}\right) u s\left(y^{j} x^{k} x^{l}\right)_{\overrightarrow{j k l}} \\
& +\frac{1}{u^{3}}\left(s^{2} Q_{s s s}+s Q_{s s}-Q_{s}\right)^{2}\left(x^{j} y^{k} y^{l}\right)_{\overrightarrow{j k l}} \\
& -\frac{s}{u^{2}} Q_{s s s} r^{2}\left(x^{j} x^{l} y^{k}\right)_{\overrightarrow{j k l}}+\frac{1}{u} Q_{s s s} r^{2} x^{j} x^{k} x^{l} \\
& +\frac{1}{u^{2}}\left(s^{2} Q_{s s}-s Q_{s}\right) r^{2}\left(\delta_{j k} y^{l}\right)_{\overrightarrow{j k l}} \\
& +\frac{1}{u^{4}}\left(3 s Q_{s}-3 s^{2} Q_{s s}-s^{3} Q_{s s s}\right) r^{2} y^{j} y^{k} y^{l},
\end{aligned}
$$

reagrupando a expressão anterior temos,

$$
\begin{aligned}
x^{i} B_{j k l}^{i}= & \frac{1}{u}\left(3 P_{s s}+s P_{s s s}+r^{2} Q_{s s s}\right) x^{j} x^{k} x^{l} \\
& +\frac{1}{u}\left(P-s P_{s}-s^{2} P_{s s}+r^{2}\left(Q_{s}-s Q_{s s}\right)\right)\left(x^{j} \delta_{k l}\right)_{\overrightarrow{j k l}} \\
& +\frac{1}{u^{2}}\left(-2 s P_{s s}-s P_{s s}-s^{2} P_{s s s}-s r^{2} Q_{s s s}\right)\left(x^{j} x^{k} y^{l}\right)_{\overrightarrow{j k l}} \\
& +\frac{1}{u^{3}}\left(s^{2} P_{s s}+s P_{s}-P+r^{2}\left(s^{2} Q_{s s s}+s Q_{s s}-Q_{s}\right)+s^{3} P_{s s s}+3 s^{2} P_{s s}\right)\left(x^{j} y^{k} y^{l}\right)_{\overrightarrow{j k l}} \\
& +\frac{1}{u^{2}}\left(s^{3} P_{s s}+s^{2} P_{s}-s P+r^{2}\left(s^{2} Q_{s s}-s Q_{s}\right)\right)\left(\delta_{j k} y^{l}\right)_{\overrightarrow{j k l}} \\
& +\frac{1}{u^{4}}\left(3 s P-s^{4} P_{s s s}-6 s^{3} P_{s s}-3 s^{2} P_{s}+r^{2}\left(3 s Q_{s}-3 s^{2} Q_{s s}-s^{3} Q_{s s s}\right)\right) y^{j} y^{k} y^{l} .
\end{aligned}
$$

Lembrando que

$$
F_{y^{i}}=\frac{1}{u}\left(\phi-s \phi_{s}\right) y^{i}+\phi_{s} x^{i}
$$

e usando (3.6) e (3.7) temos que as métricas de Landsberg esfericamente simétricas caracterizadas pela equação

$$
L_{j k l}=-\frac{1}{2} F F_{y^{i}} B_{j k l}^{i}=0
$$


são reduzidas à seguinte expressão:

$$
\begin{aligned}
F_{y^{i}} B_{j k l}^{i}= & \frac{1}{u}\left(\left(\phi-s \phi_{s}\right) P_{s s s}+3 \phi_{s} P_{s s}+s\left(\phi-s \phi_{s}\right) Q_{s s s}+s \phi_{s} P_{s s s}+r^{2} \phi_{s} Q_{s s s}\right) x^{j} x^{k} x^{l} \\
& +\frac{1}{u}\left(\left(\phi-s \phi_{s}\right)\left(-s P_{s s}+s Q_{s}-s^{2} Q_{s s}\right)+\phi_{s}\left(P-s P_{s}-s^{2} P_{s s}+r^{2}\left(Q_{s}-s Q_{s s}\right)\right)\right)\left(x^{j} \delta_{k l}\right)_{\overrightarrow{j k l}} \\
& +\frac{1}{u^{2}}\left(\left(\phi-s \phi_{s}\right)\left(-s P_{s s s}-s^{2} Q_{s s s}\right)+\phi_{s}\left(-3 s P_{s s}-s^{2} P_{s s s}-s r^{2} Q_{s s s}\right)\right)\left(x^{j} x^{k} y^{l}\right)_{\overrightarrow{j k l}} \\
& +\frac{1}{u^{3}}\left(\left(\phi-s \phi_{s}\right)\left(s P_{s s}+s^{2} P_{s s s}+s^{3} Q_{s s s}+s^{2} Q_{s s}-s Q_{s}\right)+\right. \\
& \left.\quad+\phi_{s}\left(s^{2} P_{s s}+s P_{s}-P+r^{2}\left(s^{2} Q_{s s s}+s Q_{s s}-Q_{s}\right)+s^{3} P_{s s s}+3 s^{2} P_{s s}\right)\right)\left(x^{j} y^{k} y^{l}\right)_{\overrightarrow{j k l}} \\
& +\frac{1}{u^{2}}\left(\left(\phi-s \phi_{s}\right)\left(s^{2} P_{s s}+s^{3} Q_{s s}-s^{2} Q_{s}\right)\right. \\
& \left.\quad+\phi_{s}\left(s^{3} P_{s s}+s^{2} P_{s}-s P+r^{2}\left(s^{2} Q_{s s}-s Q_{s}\right)\right)\right)\left(\delta_{j k} y^{l}\right)_{\overrightarrow{j k l}} \\
& +\frac{1}{u^{4}}\left(\left(\phi-s \phi_{s}\right)\left(-3 s^{2} P_{s s}-s^{3} P_{s s s}+3 s^{2} Q_{s}-3 s^{3} Q_{s s}-s^{4} Q_{s s s}\right)\right. \\
& \left.\quad+\phi_{s}\left(3 s P-s^{4} P_{s s s}-6 s^{3} P_{s s}-3 s^{2} P_{s}+r^{2}\left(3 s Q_{s}-3 s^{2} Q_{s s}-s^{3} Q_{s s s}\right)\right)\right) y^{j} y^{k} y^{l} .
\end{aligned}
$$

Finalmente, obtemos

$$
\begin{aligned}
F_{y^{i}} B_{j k l}^{i}= & \frac{1}{u}\left(3 \phi_{s} P_{s s}+\phi P_{s s s}+\left(s \phi+\left(r^{2}-s^{2}\right) \phi_{s}\right) Q_{s s s}\right) x^{j} x^{k} x^{l} \\
& +\frac{1}{u}\left(-s \phi P_{s s}+\phi_{s}\left(P-s P_{s}\right)+\left(s \phi+\left(r^{2}-s^{2}\right) \phi_{s}\right)\left(Q_{s}-s Q_{s s}\right)\right)\left(x^{j} \delta_{k l}\right)_{\overrightarrow{j k l}} \\
& -\frac{1}{u^{2}} s\left(3 \phi_{s} P_{s s}+\phi P_{s s s}+\left(s \phi+\left(r^{2}-s^{2}\right) \phi_{s}\right) Q_{s s s}\right)\left(x^{j} x^{k} y^{l}\right)_{\overrightarrow{j k l}} \\
& +\frac{1}{u^{3}} s^{2}\left(3 \phi_{s} P_{s s}+\phi P_{s s s}+\left(s \phi+\left(r^{2}-s^{2}\right) \phi_{s}\right) Q_{s s s}\right)\left(x^{j} y^{l} y^{l}\right)_{\overrightarrow{j k l}} \\
& -\frac{1}{u^{3}}\left(-s \phi P_{s s}+\phi_{s}\left(P-s P_{s}\right)+\left(s \phi+\left(r^{2}-s^{2}\right) \phi_{s}\right)\left(Q_{s}-s Q_{s s}\right)\right)\left(x^{j} y^{l} y^{l}\right)_{\overrightarrow{j k l}} \\
& -\frac{1}{u^{2}} s\left(-s \phi P_{s s}+\phi_{s}\left(P-s P_{s}\right)+\left(s \phi+\left(r^{2}-s^{2}\right) \phi_{s}\right)\left(Q_{s}-s Q_{s s}\right)\right)\left(\delta_{j k} y^{l}\right)_{\overrightarrow{j k l}} \\
& -\frac{1}{u^{4}} s^{3}\left(3 \phi_{s} P_{s s}+\phi P_{s s s}+\left(s \phi+\left(r^{2}-s^{2}\right) \phi_{s}\right) Q_{s s s}\right) y^{j} y^{k} y^{l} \\
& +\frac{1}{u^{4}} 3 s\left(-s \phi P_{s s}+\phi_{s}\left(P-s P_{s}\right)+\left(s \phi+\left(r^{2}-s^{2}\right) \phi_{s}\right)\left(Q_{s}-s Q_{s s}\right)\right) y^{j} y^{k} y^{l},
\end{aligned}
$$

o que finaliza a demonstração.

Proposição 3.2.2. Seja $F=|y| \phi(r, s)$ uma métrica de Landsberg sobre $M_{s}^{n} \subset \mathbb{R}^{n}$, então existem funções $f(r), c_{1}(r), c_{2}(r), g(r)$ tais que os coeficientes geodésicos $G^{i}$ satisfazem

$$
G^{i}=|y| P y^{i}+|y|^{2} Q x^{i},
$$

onde

$$
P=c_{1}(r) s+c_{2}(r) \frac{\sqrt{r^{2}-s^{2}}}{r^{2}}
$$


e

$$
Q=\frac{1}{2} f(r) s^{2}-c_{2}(r) \frac{s \sqrt{r^{2}-s^{2}}}{r^{4}}+g(r)
$$

Demonstração. Da Proposição 3.2.1, considerando $j=k=l$, como na demonstração do Teorema 2.1.2, podemos concluir que $F(x, y)=|y| \phi(r, s)$ é do tipo Landsberg se, e somente se,

$$
\begin{aligned}
& L_{1}:=3 \phi_{s} P_{s s}+\phi P_{s s s}+\left(s \phi+\left(r^{2}-s^{2}\right) \phi_{s}\right) Q_{s s s}=0 \\
& L_{2}:=-s \phi P_{s s}+\phi_{s}\left(P-s P_{s}\right)+\left(s \phi+\left(r^{2}-s^{2}\right) \phi_{s}\right)\left(Q_{s}-s Q_{s s}\right)=0,
\end{aligned}
$$

Sejam $\Theta:=P-s P_{s}$ e $\Lambda:=s \phi+\left(r^{2}-s^{2}\right) \phi_{s}$, então

$$
\Theta_{s}=-s P_{s s}, \quad \Lambda_{s}=\phi-s \phi_{s}+\left(r^{2}-s^{2}\right) \phi_{s s} .
$$

Da definição de $L_{2}$, temos que,

$$
(\phi \Theta)_{s}+\Lambda\left(Q_{s}-s Q_{s s}\right)=0 .
$$

Derivando (3.10) em relação a $s$ obtemos,

$$
(\phi \Theta)_{s s}=s \Lambda Q_{s s s}-\Lambda_{s}\left(Q_{s}-s Q_{s s}\right) .
$$

Por outro lado, da definição de $\Theta$,

$$
\begin{aligned}
(\phi \Theta)_{s s} & =\left(\phi_{s}\left(P-s P_{s}\right)-\phi\left(s P_{s s}\right)\right)_{s} \\
& =\phi_{s s} \Theta-2 s \phi_{s} P_{s s}-\phi\left(P_{s s}+s P_{s s s}\right) .
\end{aligned}
$$

De (3.8) e das duas identidades acima,

$$
\begin{aligned}
(\phi \Theta)_{s s} & =-3 s \phi_{s} P_{s s}-s \phi P_{s s s}-\Lambda_{s}\left(Q_{s}-s Q_{s s}\right) \\
-\phi\left(P_{s s}+s P_{s s s}\right)-2 s \phi_{s} P_{s s}+\phi_{s s} \Theta & =-3 s \phi_{s} P_{s s}-s \phi P_{s s s}-\Lambda_{s}\left(Q_{s}-s Q_{s s}\right)
\end{aligned}
$$

o que implica,

$$
\begin{aligned}
\Lambda_{s}\left(Q_{s}-s Q_{s s}\right) & =\left(\phi-s \phi_{s s}\right) P_{s s}-\phi_{s s} \Theta \\
& =-\frac{\phi_{s}-s \phi_{s}}{s} \Theta_{s}-\phi_{s s} \Theta .
\end{aligned}
$$

Pela Equação (3.10), temos,

$$
\Lambda\left(\phi_{s s} \Theta+\frac{\phi-s \phi_{s}}{s} \Theta_{s}\right)-\Lambda_{s}\left(\phi_{s} \Theta+\phi \Theta_{s}\right)=0 .
$$


Segue de (3.8) e (3.9) que o caso $0=\Theta=P-s P_{s}$ implica que $P$ e $Q$ são dados por (3.3), portanto $F(x, y)=|y| \phi(r, s)$ é do tipo Berwald.

Assim, considerando $\Theta \neq 0$, temos

$$
\frac{\Theta_{s}}{\Theta}\left[\left(\phi-s \phi_{s}\right) \Lambda-s \phi \Lambda_{s}\right]=s \phi_{s} \Lambda_{s}-s \phi_{s s} \Lambda .
$$

Desenvolvendo os dois lados da equação (3.11), temos

$$
\begin{aligned}
\left(\phi-s \phi_{s}\right) \Lambda-s \phi \Lambda_{s} & =\left(\phi-s \phi_{s}\right)\left(s \phi+\left(r^{2}-s^{2}\right) \phi_{s}\right)-s \phi\left[\phi-s \phi_{s}+\left(r^{2}-s^{2}\right) \phi_{s s}\right] \\
& =\left(r^{2}-s^{2}\right)\left[\left(\phi-s \phi_{s}\right) \phi_{s}-s \phi \phi_{s s}\right]
\end{aligned}
$$

e

$$
\begin{aligned}
s \phi_{s} \Lambda_{s}-s \phi_{s s} \Lambda & =s \phi_{s}\left[\phi-s \phi_{s}+\left(r^{2}-s^{2}\right) \phi_{s s}\right] \\
& =s\left(\phi-s \phi_{s}\right) \phi_{s}-s^{2} \phi \phi_{s s} .
\end{aligned}
$$

Voltando para a Equação (3.11), temos

$$
\frac{\Theta_{s}}{\Theta}\left(r^{2}-s^{2}\right)\left[\left(\phi-s \phi_{s}\right) \phi_{s}-s \phi \phi_{s s}\right]=s\left[\left(\phi-s \phi_{s}\right) \phi_{s}-s \phi \phi_{s s}\right] .
$$

Então (3.12) é satisfeita se, e somente se,

$$
\left(\phi-s \phi_{s}\right) \phi_{s}-s \phi \phi_{s s}=0
$$

$\mathrm{ou}$

$$
\frac{\Theta_{s}}{\Theta}\left(r^{2}-s^{2}\right)=s
$$

No caso da Equação (3.13):

$$
\left[\left(\phi-s \phi_{s}\right) \phi\right]_{s}=0
$$

tem como solução

$$
\phi=\sqrt{c_{1}(r) s^{2}+c_{2}(r)},
$$

onde $c_{1}(r)$ e $c_{2}(r)$ são funções de $r=|x|$. Por isso, a métrica $F=|y| \phi(r, s)$ é da forma

$$
F(x, y)=\sqrt{c_{1}(|x|)<x, y>+c_{2}(|x|)}
$$

portanto Riemanniana.

No caso da equação (3.14), temos

$$
\Theta=P-s P_{s}=\frac{c_{2}(r)}{\sqrt{r^{2}-s^{2}}}
$$


onde $c_{2}$ é uma função de $r$. Isto implica

$$
P=c_{1}(r) s+c_{2}(r) \frac{\sqrt{r^{2}-s^{2}}}{r^{2}}
$$

onde $c_{1}$ e $c_{2}$ são funções de $r$. Assim, usando a Equação (3.9), temos

$$
\begin{aligned}
\left(Q_{s}-s Q_{s s}\right)\left(s \phi+\left(r^{2}-s^{2}\right) \phi_{s}\right) & =-\frac{c_{2}(r) s}{\left(\sqrt{r^{2}-s^{2}}\right)^{3}} \phi-\frac{c_{2}(r)}{\sqrt{r^{2}-s^{2}}} \phi_{s} \\
& =-\frac{c_{2}(r)}{\left(\sqrt{r^{2}-s^{2}}\right)^{3}}\left(s \phi+\left(r^{2}-s^{2}\right) \phi_{s}\right) .
\end{aligned}
$$

Das condições (1.65) e (1.66), note que $F=|y| \phi(r, s)$ não define uma métrica de Finsler se $s \phi+\left(r^{2}-s^{2}\right) \phi_{s}=0$. Então de (3.15), $Q$ tem que satisfazer

$$
Q_{s}-s Q_{s s}=\frac{c_{2}(r)}{\left(\sqrt{r^{2}-s^{2}}\right)^{3}}
$$

Resolvendo esta equação, temos

$$
Q=\frac{1}{2} f(r) s^{2}-\frac{c_{2}(r) s \sqrt{r^{2}-s^{2}}}{r^{4}}+g(r),
$$

o que conclui a demonstração.

Teorema 3.2.3. Seja $F=|y| \phi(r, s)$ uma métrica de Finsler esfericamente simétrica sobre $M_{s}^{n} \subset \mathbb{R}^{n}$, então $F$ é do tipo Landsberg se, e somente se, $F$ é do tipo Berwald.

Demonstração. Introduzimos $U$ e $W$ como

$$
U:=\frac{s \phi+\left(r^{2}-s^{2}\right) \phi_{s}}{\phi}, \quad W:=\frac{s \phi_{r}+r \phi_{s}}{\phi} .
$$

Podemos obter $\phi_{s}$ e $\phi_{r}$ em termos de $U$ e $W$ :

$$
\phi_{s}=\frac{U-s}{r^{2}-s^{2}} \phi, \quad \phi_{r}=\frac{1}{s}\left(W-\frac{r(U-s)}{r^{2}-s^{2}}\right) \phi .
$$

Substituindo $\phi_{r}$ e $\phi_{s}$ em $P$ e $Q$, que foram definidos no Lema 1.7.1, temos

$$
\begin{aligned}
& P=\frac{W}{2 r}-Q U \\
& Q=\frac{1}{2 r s} \frac{\left(r^{2}-s^{2}\right)\left(s W_{s}-2 W\right)+(U-s)(2 r+s W)}{U^{2}-s U+\left(r^{2}-s^{2}\right) U_{s}} .
\end{aligned}
$$

De (3.17),

$$
\begin{aligned}
W & =2 r(P+U Q), \\
W_{s} & =2 r\left(P_{s}+U_{s} Q+U Q_{s}\right)
\end{aligned}
$$


substituindo (3.19) e (3.20) em (3.18),

$$
U=\frac{\left(r^{2}-s^{2}\right)\left(s P_{s}-2 P\right)-s(1+s P)}{\left(r^{2}-s^{2}\right)\left(2 Q-s Q_{s}\right)-s P-1} .
$$

Usando os dados da Proposição 3.2.2 em (3.17) e (3.18) temos,

$$
\begin{aligned}
U & =\frac{r^{2}\left(s+c_{1}(r) r^{2} s+2 c_{2}(r) \sqrt{r^{2}-s^{2}}\right)}{r^{2}+2 c_{2}(r) s \sqrt{r^{2}-s^{2}}+c_{1}(r) r^{2} s^{2}-2 g(r) r^{2}\left(r^{2}-s^{2}\right)} \\
W & =2 r(P+U Q) .
\end{aligned}
$$

De (3.16), podemos observar que $\phi$ tem que satisfazer

$$
\begin{aligned}
(\ln \phi)_{s} & =\frac{\left(c_{1}(r)+2 c_{2}(r)\right) r^{2} s+2 c_{2}(r) \sqrt{r^{2}-s^{2}}}{r^{2}+\left(c_{1}(r)+2 g(r)\right) r^{2} s^{2}-2 c_{3}(r) r^{4}+2 c_{2}(r) s \sqrt{r^{2}-s^{2}}} \\
(\ln \phi)_{r} & =\frac{\sqrt{r^{2}-s^{2}}\left(4 f(r) c_{2}(r) r^{4} s^{2}+4 c_{1}(r) c_{2}(r) r^{2} s^{2}-2 c_{2}(r) s^{2}+4 c_{2}(r) g(r)\right)}{r s\left(r^{2}+\left(c_{1}(r)+2 g(r)\right) r^{2} s^{2}-2 c_{3}(r) r^{4}+2 c_{2}(r) s \sqrt{r^{2}-s^{2}}\right)} \\
& +\frac{2 f(r) c_{1}(r) r^{6} s^{3}+f(r) r^{4} s^{3}+c_{1}(r) r^{4} s+4 c_{1}(r) c_{3}(r) r^{4} s^{3}+2 c_{1}^{2}(r) r^{4} s^{3}-2 c_{1}(r) g(r) r^{6} s}{r s\left(r^{2}+\left(c_{1}(r)+2 g(r)\right) r^{2} s^{2}-2 c_{3}(r) r^{4}+2 c_{2}(r) s \sqrt{r^{2}-s^{2}}\right)} .
\end{aligned}
$$

O sistema (3.24)-(3.25) tem solução se, e somente se,

$$
(\ln \phi)_{s r}=(\ln \phi)_{r s}
$$

isto é,

$$
\begin{aligned}
0= & \frac{1}{\sqrt{r^{2}-s^{2}} r s^{2}\left(r^{2} s^{2} c_{1}-2 r^{4} g+2 r^{2} s^{2} g+2 c_{2} s \sqrt{r^{2}-s^{2}}+r^{2}\right)^{2}}\left[\sqrt { r ^ { 2 } - s ^ { 2 } } \left(-2 c_{1}^{2} r^{6} s^{3}+8 g^{2} r^{6} s^{3}\right.\right. \\
& c_{1}^{\prime} r^{5} s^{3}+2 g r^{5} s^{3}+8 c_{1} g f r^{10} s^{3}-16 c_{2}^{2} g s^{3}-4 c_{1} f r^{8} s^{3}+4 c_{1}^{2} g r^{8} s^{3}+8 c_{1} g^{2} r^{8} s^{3}-2 c_{1} g r^{7} s^{3} \\
& \left.+2 g^{\prime} c_{1} r^{7} s^{3}+16 c_{2}^{2} g r^{2} s+8 g f r^{8} s^{3}-4 f r^{6} s^{3}\right)+2 c_{1} c_{2} r^{4} s^{4}+8 g c_{2} r^{6} s^{2}-12 g c_{2} r^{4} s^{4}+24 g^{2} c_{2} r^{4} s^{2} \\
& -16 g^{2} c_{2} r^{2} s^{4}-4 c_{2} f r^{8} s^{2}+2 c_{2}^{\prime} r^{5} s^{2}-2 c_{2}^{\prime} r^{3} s^{4}+4 g c_{2} r^{4}-8 g^{2} c_{2} r^{6}+8 g c_{2} f r^{8} s^{2}\left(r^{2}-s^{2}\right) \\
& +4 c_{1} g c_{2} r^{8} s^{2}+12 c_{1} g c_{2} r^{4} s^{2}-8 c_{1} g c_{2} r^{2} s^{4}\left(r^{4}+1\right)+4 r^{5} s^{2}\left(g^{\prime} c_{s}-c_{2}^{\prime} g\right)\left(r^{2}-s^{2}\right) \\
& -2 c_{1} c_{2} r^{6} s^{2}+4 c_{2} f r^{6} s^{4} .
\end{aligned}
$$

Observe que uma condição para que a equação acima esteja definida em $s=0$ é

$$
c_{2}^{2}(r) g(r)=0
$$

Se $c_{2}(r)=0$, pela Proposição 3.1.2, a métrica $F(x, y)=|y| \phi(r, s)$ é do tipo Berwald. A reciproca é imediata. 
Se $c_{2}(r) \neq 0$, ou seja, $g(r)=0$. Temos que as equações (3.24)-(3.25) tornam-se

$$
\begin{aligned}
(\ln \phi)_{s}= & \frac{c 1(r) r^{2} s+2 c_{2}(r) \sqrt{r^{2}-s^{2}}}{r^{2}+c_{1}(r) r^{2} s^{2}+2 c_{2}(r) s \sqrt{r^{2}-s^{2}}} \\
(\ln \phi)_{r}= & \frac{\sqrt{r^{2}-s^{2}}\left(4 c_{0}(r) c_{2}(r) r^{4} s+4 c_{1}(r) c_{2}(r) r^{2} s-2 c_{2}(r) s\right)}{r\left(r^{2}+c_{1}(r) r^{2} s^{2}+2 c_{2}(r) s \sqrt{r^{2}-s^{2}}\right)} \\
& +\frac{2 f(r) c_{1}(r) r^{6} s^{2}+2 f(r) r^{4} s^{2}+c_{1}(r) r^{4}+2 c_{1}^{2}(r) r^{4} s^{2}}{r\left(r^{2}+c_{1}(r) r^{2} s^{2}+2 c_{2}(r) s \sqrt{r^{2}-s^{2}}\right)} .
\end{aligned}
$$

Isto simplifica o cálculo de $(\ln \phi)_{s r}$ e $(\ln \phi)_{r s}$

$$
(\ln \phi)_{s r}=-\frac{r\left[\left(2 c_{2}^{\prime}(r) r-4 c_{2}(r)\right) s^{2}+2 c_{2}(r) r^{2}-2 c_{2}^{\prime} r^{3}-c_{1}^{\prime}(r) \sqrt{r^{2}-s^{2}} r^{3} s\right]}{\sqrt{r^{2}-s^{2}}\left[r^{2}+2 c_{2}(r) s \sqrt{r^{2}-s^{2}}+\left(c_{1}(r)+2 g(r)\right) r^{2} s^{2}-2 g(r) r^{4}\right]^{2}}
$$

e

$$
\begin{aligned}
(\ln \phi)_{r s}= & -\frac{2 r\left[\left(2 f(r) c_{2}(r) r^{4}+c_{1}(r) c_{2}(r) r^{2}-2 c_{2}(r)\right) s^{2}+c_{2}(r) r^{2}-c_{1}(r) c_{2}(r) r^{4}\right]}{\sqrt{r^{2}-s^{2}}\left[r^{2}+2 c_{2}(r) s \sqrt{r^{2}-s^{2}}+\left(c_{1}(r)+2 g(r)\right) r^{2} s^{2}-2 g(r) r^{4}\right]^{2}} \\
& +\frac{2 r\left[-2 f(r) c_{2}(r) r^{6}-s r^{4} \sqrt{r^{2}-s^{2}}\left(2 f(r) c_{1}(r) r^{2}+c_{1}^{2}(r)+2 f(r)\right)\right]}{\sqrt{r^{2}-s^{2}}\left[r^{2}+2 c_{2}(r) s \sqrt{r^{2}-s^{2}}+\left(c_{1}(r)+2 g(r)\right) r^{2} s^{2}-2 g(r) r^{4}\right]^{2}}
\end{aligned}
$$

Logo, de $(\ln \phi)_{r s}=(\ln \phi)_{s r}$, temos

$$
\begin{aligned}
r\left[2 c_{2}^{\prime}(r) r-4 c_{2}(r)\right] s^{2} & =2 r\left[2 f(r) c_{2}(r) r^{4}+c_{1}(r) c_{2}(r) r^{2}-2 c_{2}(r)\right] s^{2} \\
-r\left[c_{1}^{\prime}(r) r^{3}\right] s & =-2 s r^{5}\left[2 f(r) c_{1}(r) r^{2}+c_{1}^{2}(r)+2 f(r)\right] \\
r\left[2 c_{2}(r) r^{2}-2 c_{2}^{\prime}(r) r^{3}\right] & =2 r\left[c_{2}(r) r^{2}-c_{1}(r) c_{2}(r) r^{4}-2 f(r) c_{2}(r) r^{6}\right] .
\end{aligned}
$$

As equações acima são equivalente a

$$
\begin{array}{r}
c_{1}^{\prime}-4 f(r) c_{1}(r) r^{3}-2 c_{1}^{2}(r)-4 f(r) r=0 \\
c_{2}^{\prime}(r)-2 f(r) c_{2}(r) r^{3}-c_{1}(r) c_{2}(r) r=0 .
\end{array}
$$

Eliminando $c_{0}(r)$, obtemos que $c_{1}(r)$ e $c_{2}(r)$ devem satisfazer

$$
c_{2}(r) c_{1}^{\prime}(r)+\left(\frac{2}{r} c_{2}(r)-2 c_{2}^{\prime}(r)\right) c_{1}(r)-\frac{2}{r^{2}} c_{2}^{\prime}(r)=0 .
$$

Resolvendo a equação em $c_{1}(r)$, podemos obter que

$$
c_{1}(r)=\frac{1}{r^{2}}+a \frac{c_{2}^{2}(r)}{r^{2}},
$$

onde $a$ é uma constante. Substituindo $c_{1}(r)$ em (3.26) e resolvendo esta, obtemos que

$$
\phi=\frac{\sqrt{r^{2}-s^{2}+2 c_{2} \sqrt{r^{2}-s^{2}} s+a c_{2}^{2} s^{2}}}{r} \exp \left(\frac{1}{\sqrt{1-a}}\left(\operatorname{arctanh}\left(\frac{\sqrt{r^{2}-s^{2}}+c_{2} s}{\sqrt{1-a} c_{2} s}\right)+c\right)\right)
$$


quando $a<1$ ou

$$
\phi=\frac{\sqrt{r^{2}-s^{2}+2 c_{2} \sqrt{r^{2}-s^{2}} s+a c_{2}^{2} s^{2}}}{r} \exp \left(-\frac{1}{\sqrt{a-1}}\left(\arctan \left(\frac{\sqrt{r^{2}-s^{2}}+c_{2} s}{\sqrt{a-1} c_{2} s}\right)+c\right)\right)
$$

quando $a>1$. Aqui $c$ é uma função de $r$.

Como $F(r, s)=|y| \phi(r, s)$ é uma métrica de Finsler e tem que estar definida em todo $T\left(M_{s}^{n}\right)$ (em particular para $s \rightarrow 0$ ), a primeira solução é descartada. Derivando duas vezes a segunda solução em relação a $s$ obtemos

$$
\begin{aligned}
\phi_{s s}= & {\left[\frac{a^{3} c_{2}^{6} s^{4}+c_{2}^{4}\left(a^{2} s^{2}\left(2 r^{2}-3 s^{2}\right)+4 a s^{2}\left(r^{2}-3 s^{2}\right)\right)+c_{2}^{2}\left(r^{2}-s^{2}\right)\left(a\left(r^{2}-3 s^{2}\right)-12 s^{2}\right)-\left(r^{2}-s^{2}\right)^{2}}{\left(r^{2}-s^{2}+2 s c_{2} \sqrt{r^{2}-s^{2}}+a s^{2} c_{2}^{2}\right)^{\frac{7}{2}}}\right.} \\
& \left.+\frac{2 c_{2}^{5} a^{2} s^{3}\left(2 r^{2}-3 s^{2}\right)+4 c_{2}^{3} s\left(a r^{2}\left(r^{2}-4 s^{2}\right)+3 a s^{4}-2 s^{2}\left(r^{2}-s^{2}\right)\right)-6 c_{2} s\left(r^{2}-s^{2}\right)^{2}}{\sqrt{r^{2}-s^{2}}\left(r^{2}-s^{2}+2 s c_{2} \sqrt{r^{2}-s^{2}}+a s^{2} c_{2}^{2}\right)^{\frac{7}{2}}}\right] \times \\
& \exp \left(-\frac{1}{\sqrt{a-1}}\left(\arctan \left(\frac{\sqrt{r^{2}-s^{2}}+c_{2} s}{\sqrt{a-1} c_{2} s}\right)+c\right)\right) .
\end{aligned}
$$

e observamos que $\phi$ não é contínua ao longo de $s=r$ e é descartada também. 


\section{MÉTRICAS DE DOUGLAS COM S- CURVATURA NULA}

\subsection{Métricas de Douglas esfericamente simétrica com S-curvatura nula}

Nesta seção discutiremos as condições necessárias e suficientes para que uma métrica de Douglas esfericamente simétrica tenha $S$-curvatura nula.

Seja $A(r)$ uma função positiva que define o elemento de volume de uma métrica esfericamente simétrica, isto é:

$$
A(r):= \begin{cases}\frac{\int_{0}^{\pi} \operatorname{sen}^{n-2}(t) d t}{\int_{0}^{\pi} \frac{\sin ^{n-2}(t)}{\phi(r, r \cos (t))^{n}} d t} & \text { se } d V=d V_{B H} \\ \frac{\int_{0}^{\pi}\left(\operatorname{sen}^{n-2}(t)\right) T(r, r \cos (t)) d t}{\int_{0}^{\pi} \operatorname{sen}^{n-2}(t) d t} & \text { se } d V=d V_{H T},\end{cases}
$$

onde $T(r, s):=\phi\left(\phi-s \phi_{s}\right)^{n-2}\left[\left(\phi-s \phi_{s}\right)+\left(r^{2}-s^{2}\right) \phi_{s s}\right]$.

De agora em diante só usaremos a expressão de $d V_{B H}$, salvo menção contrária.

Observe que, como $r=|x|$, segue que

$$
\frac{y^{m}}{A(r)} \frac{\partial A(r)}{\partial x^{m}}=\frac{A^{\prime}(r)}{A(r)} \frac{x^{m} y^{m}}{r}=|y| \frac{s}{r} \frac{A^{\prime}(r)}{A(r)},
$$

e da definição de $S$-curvatura dada em (1.37), e usando (2.19) temos

$$
S=|y|\left[(n+1) P+2 s Q+\left(r^{2}-s^{2}\right) Q_{s}\right]-|y| \frac{s}{r} \frac{A^{\prime}(r)}{A(r)},
$$

onde $r, s, Q$ e $P$ foram definidos em (1.49) e (1.50).

Note que, se $F$ é de Douglas, então segue da Proposição 2.1.2 que $Q=g(r)+\frac{s^{2}}{2} f(r)$. Assim, podemos obter a seguinte Proposição.

Proposição 4.1.1. Seja $F=|y| \phi\left(|x|, \frac{\langle x, y\rangle}{|y|}\right)$ uma métrica de Douglas esfericamente simétrica sobre $M_{s}^{n} \in \mathbb{R}^{n}$. Então a $S$-curvatura de $F$ é dada por

$$
\begin{aligned}
\frac{2 r}{n+1} \phi \mathrm{S}=|y|\left\{\left[1-\left(r^{2}-s^{2}\right)(2 g(r)\right.\right. & \left.\left.+s^{2} f(r)\right)\right] r \phi_{s}+s \phi_{r}-s r\left(2 g+s^{2} f(r)\right) \phi \\
& \left.-s\left[-\frac{2 r}{n+1}\left(2 g(r)+r^{2} f(r)\right)+\frac{2}{n+1} \frac{A^{\prime}(r)}{A(r)}\right] \phi\right\} .
\end{aligned}
$$


Demonstração. A demonstração segue diretamente da substituição de $Q=g(r)+\frac{s^{2}}{2} f(r)$ em (4.2), isto é,

$$
\begin{aligned}
S & =|y|\left\{(n+1) P+2 s g(r)+s^{3} f(r)+r^{2} s f(r)-s^{3} f(r)-\frac{s}{r} \frac{A^{\prime}(r)}{A(r)}\right\} \\
& =|y|\left\{\frac{n+1}{2 r \phi}\left(r \phi_{s}+s \phi_{r}-2 r Q\left(s \phi+\left(r^{2}-s^{2}\right) \phi_{s}\right)\right)+s\left(2 g(r)+r^{2} f(r)\right)-\frac{s}{r} \frac{A^{\prime}(r)}{A(r)}\right\},
\end{aligned}
$$

que é equivalente a (4.3).

Uma classe interessante de métricas de Douglas esfericamente simétricas é quando a $S$-curvatura é nula. Isto nos leva ao seguinte teorema de caracterização de tais métricas, cuja demonstração segue diretamente da proposição anterior e do Teorema 2.2.1.

Teorema 4.1.2. Seja $F=|y| \phi\left(|x|, \frac{\langle x, y\rangle}{|y|}\right)$ uma métrica esfericamente simétrica sobre $M_{s}^{n} \subset \mathbb{R}^{n}$. Então $F$ é de Douglas com $S$-curvatura nula se, e somente se, $\phi$ satisfazo seguinte sistema de EDP,

$$
\begin{array}{r}
{\left[\left(r^{2}-s^{2}\right)\left(2 g+f s^{2}\right)-1\right] r \phi_{s s}-s \phi_{r s}+\phi_{r}+r\left(2 g+f s^{2}\right)\left(\phi-s \phi_{s}\right)=0,} \\
{\left[1-\left(r^{2}-s^{2}\right)\left(2 g+f s^{2}\right)\right] r \phi_{s}+s \phi_{r}-r s\left(2 g+s^{2} f\right) \phi} \\
-\frac{2 s}{n+1}\left(\frac{A_{\phi}^{\prime}(r)}{A_{\phi}(r)}-r\left(2 g+r^{2} f\right)\right) \phi=0 .
\end{array}
$$

para um par de funções diferenciáveis $f=f(r)$ e $g=g(r)$.

Observação 4.1.1. Observe que este último teorema e o Teorema 3.1.3 nos diz que toda métrica de Douglas esfericamente simétrica sobre $M_{s}^{n} \in \mathbb{R}^{n}$ com $S$-curvatura nula é do tipo Berwald, isto é, bastaria considerar em (3.5)

$$
c_{1}(r)=\frac{1}{r(n+1)}\left(\frac{A_{\phi}^{\prime}(r)}{A_{\phi}(r)}-r\left(2 g+r^{2} f\right)\right) .
$$

Por outro lado, a Observação 1.5.1 juntamente com a Proposição 1.6.2 nos diz que toda métrica de Berwald tem $S$-curvatura nula e é do tipo Douglas.

Desta observação obtemos este interessante resultado:

Teorema 4.1.3. Seja $F=|y| \phi(r, s)$ uma métrica de Finsler esfericamente simétrica definida sobre $M_{s}^{n} \in \mathbb{R}^{n}$, então $F$ é do tipo Douglas com $S$-curvatura nula se, e somente se, F é do tipo Berwald.

Observação 4.1.2. O Teorema 4.1.3 continua valendo para uma forma de volume HolmesThompson como veremos na Observação 4.2.4. 


\subsection{Caracterização em termos de Métricas de Berwald e de Lands- berg}

Em virtude do Teorema 4.1.3 o problema de solucionar o sistema (4.4)-(4.5) que envolve o elemento de volume, se reduz a tentar classificar as métricas do tipo Berwald esfericamente simétricas.

Antes de enunciar o Lema 4.2.1 precisamos de algumas condições. Sejam $f(r)$ e $g(r)$ tais que as seguintes expressões estejam bem definidas

$$
\int 2 r\left(2 g+r^{2} f\right) d r \quad \text { e } \quad \int 2 r f e^{\int 2 r\left(2 g+r^{2} f\right) d r} d r
$$

e adicionalmente tal que

$$
\left(r^{2}-s^{2}\right) \int 2 r f e^{\int 2 r\left(2 g+r^{2} f\right) d r} d r-e^{\int 2 r\left(2 g+r^{2} f\right) d r} \neq 0 \quad \forall(r, s) \in[0, \nu) \times(-\nu, \nu) .
$$

Lema 4.2.1. Seja $\phi(r, s)$ uma função sobre $I \times \mathbb{R}$ que satisfaz o seguinte sistema:

$$
\begin{gathered}
{\left[\left(r^{2}-s^{2}\right)\left(2 g+f s^{2}\right)-1\right] r \phi_{s s}-s \phi_{r s}+\phi_{r}+r\left(2 g+f s^{2}\right)\left(\phi-s \phi_{s}\right)=0} \\
{\left[1-\left(r^{2}-s^{2}\right)\left(2 g+f s^{2}\right)\right] r \phi_{s}+s \phi_{r}-s\left[r\left(2 g+s^{2} f\right)+L(r)\right] \phi=0}
\end{gathered}
$$

onde $f(r), g(r)$ e $L(r)$ são funções diferenciáveis sobre $I \in R$. Suponha que $L(r) \neq-2 / r$ e $g(r) \neq \frac{1}{2 r^{2}}$, então a menos de homotetia:

$$
\phi(r, s)=\frac{\sqrt{\left|\left(r^{2}-s^{2}\right) \int 2 r f e^{\int 2 r\left(2 g+r^{2} f\right) d r} d r-e^{\int 2 r\left(2 g+r^{2} f\right) d r \mid}\right|}}{\left|r^{2} \int 2 r f e^{\int 2 r\left(2 g+r^{2} f\right) d r} d r-e^{\int 2 r\left(2 g+r^{2} f\right) d r}\right|},
$$

ou

$$
\phi(r, s)=\frac{\sqrt{r^{2}-s^{2}}}{r^{2}}
$$

Demonstração. Defina

$$
\psi(r, s):=\frac{e^{-\int L(r) d r}}{\sqrt{r^{2}-s^{2}}} \phi(r, s) .
$$

Neste caso, temos que

$$
\begin{aligned}
\phi_{r} & =L e^{\int L d r} \sqrt{r^{2}-s^{2}} \psi+\frac{r e^{\int L d r}}{\sqrt{r^{2}-s^{2}}} \psi+e^{\int L d r} \sqrt{r^{2}-s^{2}} \psi_{r} \\
& =e^{\int L d r \sqrt{r^{2}-s^{2}}}\left(L \psi+\frac{r \psi}{r^{2}-s^{2}}+\psi_{r}\right), \\
\phi_{s} & =e^{\int L d r}\left(-\frac{s}{\sqrt{r^{2}-s^{2}} \psi+\sqrt{r^{2}-s^{2}} \psi_{s}}\right) \\
& =e^{\int L d r} \sqrt{r^{2}-s^{2}}\left(\psi_{s}-\frac{s}{r^{2}-s^{2}} \psi\right),
\end{aligned}
$$


substituindo na Equação (4.9), temos

$$
\begin{aligned}
\left.0=e^{\int L d r \sqrt{r^{2}-s^{2}}\left\{\left(1-\left(r^{2}-s^{2}\right)\right.\right.}\left(2 g+s^{2} f\right)\right) r\left[\psi_{s}-\frac{s}{r^{2}-s^{2}} \psi\right] \\
\left.+s\left[L \psi+\frac{r \psi}{r^{2}-s^{2}}+\psi_{r}\right]-s\left(r\left(2 g+s^{2} f\right)+L\right) \psi\right\},
\end{aligned}
$$

de onde obtemos,

$$
0=\left[1-\left(r^{2}-s^{2}\right)\left(2 g+s^{2} f\right)\right] r \psi_{s}+s \psi_{r}
$$

Então, segue de (4.12) que

$$
\frac{e^{-\int L(r) d r} \phi(r, s)}{\sqrt{r^{2}-s^{2}}}
$$

é uma função de $\varphi(r, s)$ definida em (1.75) (Lema 1.8.1), isto é, $\phi(r, s)$ é dado por:

$$
\phi(r, s)=e^{\int L(r) d r} \sqrt{r^{2}-s^{2}} \eta(\varphi(r, s)),
$$

onde $\eta$ é uma função diferenciável de $\varphi$.

Observamos que a equação (4.8) pode ser reescrita por

$$
\left[1-\left(r^{2}-s^{2}\right)\left(2 g+s^{2} f\right)\right] r \frac{\partial}{\partial s}\left(\left(\phi-s \phi_{s}\right) \sqrt{r^{2}-s^{2}}\right)+s \frac{\partial}{\partial r}\left(\left(\phi-s \phi_{s}\right) \sqrt{r^{2}-s^{2}}\right)=0 .
$$

De (4.14) temos:

$$
\phi-s \phi_{s}=\frac{e^{\int L d r}}{\sqrt{r^{2}-s^{2}}}\left\{r^{2} \eta(\varphi)-\frac{2 s^{2}}{r^{2}-s^{2}} e^{\int 2 r\left(2 g+r^{2} f\right) d r} \varphi^{2} \eta^{\prime}(\phi)\right\} .
$$

Substituindo a última igualdade em (4.15) e tendo em conta o Lema 1.8.1, pois $\eta(\varphi)$ e $\varphi^{2} \eta^{\prime}(\varphi)$ são funções de $\varphi$ e elas devem satisfazer a equação (1.73), temos

$$
\begin{aligned}
0=\left[2 r s+s r^{2} L\right] \eta & -\left[\left[1-\left(r^{2}-s^{2}\right)\left(2 g+s^{2} f\right)\right] r \frac{\partial}{\partial s}\left(\frac{2 s^{2}}{r^{2}-s^{2}} e^{\int 2 r\left(2 g+r^{2} f\right) d r}\right)\right. \\
& \left.+s \frac{\partial}{\partial r}\left(\frac{2 s^{2}}{r^{2}-s^{2}} e^{\int 2 r\left(2 g+r^{2} f\right) d r}\right)+s L\left(\frac{2 s^{2}}{r^{2}-s^{2}} e^{\int 2 r\left(2 g+r^{2} f\right) d r}\right)\right] \varphi^{2} \eta^{\prime} .
\end{aligned}
$$

Calculando as derivadas envolvidas na equação acima:

$$
\begin{aligned}
\frac{\partial}{\partial s}\left(\frac{2 s^{2}}{r^{2}-s^{2}} e^{\int 2 r\left(2 g+r^{2} f\right)}\right) & =\left(\frac{4 s}{r^{2}-s^{2}}+\frac{4 s^{3}}{\left(r^{2}-s^{2}\right)^{2}}\right) e^{\int 2 r\left(2 g+r^{2} f\right) d r} \\
& =\frac{4 r^{2} s}{\left(r^{2}-s^{2}\right)^{2}} e^{\int 2 r\left(2 g+r^{2} f\right) d r} \\
\frac{\partial}{\partial r}\left(\frac{2 s^{2}}{r^{2}-s^{2}} e^{\int 2 r\left(2 g+r^{2} f\right)}\right) & =\left(\frac{2 s^{2} 2 r\left(2 g+r^{2} f\right)}{r^{2}-s^{2}}-\frac{4 r s^{2}}{\left(r^{2}-s^{2}\right)^{2}}\right) e^{\int 2 r\left(2 g+r^{2} f\right) d r} \\
& =-\frac{4 r s^{2}}{\left(r^{2}-s^{2}\right)^{2}}\left(1-\left(r^{2}-s^{2}\right)\left(2 g+r^{2} f\right)\right) e^{\int 2 r\left(2 g+r^{2} f\right) d r},
\end{aligned}
$$


e substituindo na equação anterior,

$$
\begin{aligned}
{\left[2 r s+s r^{2} L\right] \eta=\varphi^{2} \eta^{\prime} } & {\left[\frac { 4 r s e ^ { \int 2 r ( 2 g + r ^ { 2 } f ) d r } } { ( r ^ { 2 } - s ^ { 2 } ) ^ { 2 } } \left\{\left[1-\left(r^{2}-s^{2}\right)\left(2 g+s^{2} f\right)\right] r^{2}\right.\right.} \\
& \left.\left.\quad-s^{2}\left[1-\left(r^{2}-s^{2}\right)\left(2 g+r^{2} f\right)\right]\right\}+s L\left(\frac{2 s^{2} e^{\int 2 r\left(2 g+r^{2} f\right) d r}}{r^{2}-s^{2}}\right)\right] .
\end{aligned}
$$

Continuando os cálculos,

$$
\begin{aligned}
\left(2 r+r^{2} L\right) \eta & =\varphi^{2} \eta^{\prime} e^{\int 2 r\left(2 g+r^{2} f\right) d r}\left(\frac{4 r\left[1-\left(r^{2}-s^{2}\right)\left(2 g+s^{2} f\right)\right]}{\left(r^{2}-s^{2}\right)^{2}}\left(r^{2}-s^{2}\right)\right. \\
& \left.+\frac{4 r s^{2} f}{\left(r^{2}-s^{2}\right)^{2}}\left(r^{2}-s^{2}\right)^{2}+\frac{2 s^{2} L}{r^{2}-s^{2}}\right) \\
& =\varphi^{2} \eta^{\prime} \frac{e^{2 r\left(2 g+r^{2} f\right) d r}}{\left(r^{2}-s^{2}\right)}\left(4 r\left[1-\left(r^{2}-s^{2}\right)\left(2 g+s^{2} f\right)\right]+\left(r^{2}-s^{2}\right) 4 r s^{2} f+2 s^{2} L\right) \\
& =\varphi^{2} \eta^{\prime} \frac{e^{\int 2 r\left(2 g+r^{2} f\right) d r}}{\left(r^{2}-s^{2}\right)}\left(4 r-4 r 2 g\left(r^{2}-s^{2}\right)+2 s^{2} L\right),
\end{aligned}
$$

assim, temos

$$
\left(r^{2}-s^{2}\right)\left[2 r+r^{2} L(r)\right] \eta(\varphi)=e^{\int 2 r\left(2 g+r^{2} f\right) d r}\left[4 r-4 r 2 g\left(r^{2}-s^{2}\right)+2 s^{2} L(r)\right] \varphi^{2} \eta^{\prime}(\varphi) .
$$

Se $n(\varphi)$ for uma constante não nula, teríamos que $L(r)=\frac{-2}{r}$, o que implicaria em

$$
\begin{aligned}
\phi(r, s) & =e^{-\int \frac{2}{r} d r} \sqrt{r^{2}-s^{2}} \\
& =\frac{c \sqrt{r^{2}-s^{2}}}{r^{2}}
\end{aligned}
$$

onde $c$ é uma constante positiva, portanto $\phi$ a menos de homotetia é dada por (4.11) . Por outro lado, se $\eta^{\prime}(\varphi) \neq 0$ e introduzindo a notação $B=B(r, s)$ definido por

$$
B(r, s):=\frac{4 r-8 r g\left(r^{2}-s^{2}\right)+2 s^{2} L(r)}{\left(r^{2}-s^{2}\right)\left(2 r+r^{2} L(r)\right)}
$$

a identidade (4.16) pode ser expressa da seguinte forma

$$
\frac{\eta(\varphi)}{\varphi^{2} \eta^{\prime}(\varphi)}=B e^{\int 2 r\left(2 g+r^{2} f\right) d r}
$$

O lado esquerdo da equação (4.18) é uma função de $\varphi$, logo podemos usar o Lema 1.8.2 para $B=B(r, s)$, isto é,

$$
0=\left[1-\left(r^{2}-s^{2}\right)\left(2 g+s^{2} f\right)\right] r \frac{\partial}{\partial s} B+s \frac{\partial}{\partial r} B+2 r s\left(2 g+r^{2} f\right) B,
$$


calculando as derivadas envolvidas na equação anterior,

$$
\begin{aligned}
\frac{\partial B}{\partial r}= & \frac{1}{\left(\left(r^{2}-s^{2}\right)\left(2 r+r^{2} L\right)\right)^{2}}\left[\left(r^{2}-s^{2}\right)\left(2 r+r^{2} L\right)\left[4-\left(4 r 2 g^{\prime}+8 g\right)\left(r^{2}-s^{2}\right)-8 r^{2} 2 g+2 s^{2} L^{\prime}\right]\right. \\
& \left.-\left(4 r-4 r 2 g\left(r^{2}-s^{2}\right)+2 s^{2} L\right)\left[2 r\left(2 r+r^{2} L\right)+\left(r^{2}-s^{2}\right)\left(2+2 r L+r^{2} L^{\prime}\right)\right]\right] \\
= & \frac{1}{\left(\left(r^{2}-s^{2}\right)\left(2 r+r^{2} L\right)\right)^{2}}\left[( r ^ { 2 } - s ^ { 2 } ) \left[\left(2 r+r^{2} L\right)\left(4-8 r^{2} 2 g+2 s^{2} L^{\prime}\right)+4 r 2 g\left(2 r\left(2 r+r^{2} L\right)\right)\right.\right. \\
& \left.-\left(2+2 r L+r^{2} L^{\prime}\right)\left(4 r+2 s^{2} L\right)\right]+\left(r^{2}-s^{2}\right)^{2}\left[-\left(2 r+r^{2} L\right)\left(4 r 2 g^{\prime}+8 g\right)\right. \\
& \left.\left.+4 r 2 g\left(2+2 r L+r^{2} L^{\prime}\right)\right]-2 r\left(4 r+2 s^{2} L\right)\left(2 r+r^{2} L\right)\right]
\end{aligned}
$$

$$
\begin{aligned}
\frac{\partial B}{\partial s}= & \frac{1}{\left(\left(r^{2}-s^{2}\right)\left(2 r+r^{2} L\right)\right)^{2}}\left[\left(r^{2}-s^{2}\right)\left(2 r+r^{2} L\right)[8 r s 2 g+4 s L]-\right. \\
& \left.-\left(4 r-4 r 2 g\left(r^{2}-s^{2}\right)+2 s^{2} L\right)\left[-2 s\left(2 r+r^{2} L\right)\right]\right] \\
= & \frac{1}{\left(\left(r^{2}-s^{2}\right)\left(2 r+r^{2} L\right)\right)^{2}}\left[\left(r^{2}-s^{2}\right) 4 s L\left(2 r+r^{2} L\right)+\left(4 r+2 s^{2} L\right) 2 s\left(2 r+r^{2} L\right)\right] \\
= & \frac{1}{\left(\left(r^{2}-s^{2}\right)\left(2 r+r^{2} L\right)\right)^{2}}\left[2 s\left(2 r+r^{2} L\right)\left(2 L\left(r^{2}-s^{2}\right)+4 r+2 s^{2} L\right)\right] \\
= & \frac{4 s}{\left(\left(r^{2}-s^{2}\right)\right.}
\end{aligned}
$$

Assim a equação (4.19) se reduz a:

$$
\begin{aligned}
0= & {\left[1-\left(r^{2}-s^{2}\right)\left(2 g+s^{2} f\right)\right] r\left[4 s\left(2 r+r^{2} L\right)^{2}\right]+s\left\{( r ^ { 2 } - s ^ { 2 } ) ^ { 2 } \left[L^{\prime}\left(4 r^{3} 2 g\right)\right.\right.} \\
& \left.+L\left(-r^{2}\left(4 r 2 g^{\prime}+8 g\right)+8 r^{2} 2 g\right)-8 r^{2} 2 g^{\prime}\right]+\left(r^{2}-s^{2}\right)\left[\left(2 r+r^{2} L\right)\left(4+2 s^{2} L^{\prime}\right)\right. \\
& \left.\left.-\left(2+2 r+r^{2} L^{\prime}\right)\left(4 r+2 s^{2} L\right)\right]-2 r\left(4 r+2 s^{2} L\right)\left(2 r+r^{2} L\right)\right\} \\
& +s 2 r\left(2 g+r^{2} f\right)\left(r^{2}-s^{2}\right)\left(2 r+r^{2} L\right)\left(4 r-8 r g\left(r^{2}-s^{2}\right)+2 s^{2} L\right) \\
= & \left(r^{2}-s^{2}\right)^{2}\left[L^{\prime}\left(4 r^{3} 2 g\right)+L\left(8 r^{2} g-8 r^{3} g^{\prime}\right)-\left(8 r^{2} 2 g^{\prime}\right)-2 r\left(2 g+r^{2} f\right)\left(2 r+r^{2} L\right) 8 r g\right] \\
& +\left(r^{2}-s^{2}\right)\left[-r\left(2 g+s^{2} f\right) 4\left(2 r+r^{2} L\right)^{2}+4 r s^{2} L^{\prime}+4 L\left(r^{2}-s^{2}\right)-2 r L\left(4 r+2 s^{2} L\right)\right. \\
& \left.-4 r^{3} L+2 r\left(2 g+r^{2} f\right)\left(2 r+r^{2} L\right)\left(4 r+2 s^{2} L\right)\right] \\
& +4 r\left(2 g+r^{2} f\right)\left(2 r+r^{2} L\right)^{2}-2 r\left(4 r+2 s^{2} L\right)\left(2 r+r^{2} L\right) \\
& \left(r^{2}-s^{2}\right)^{2}\left[L^{\prime}\left(8 r^{3} g\right)+L\left(8 r^{2} g-8 r^{3} g^{\prime}-16 r^{4}\left(2 g+r^{2} f\right) g\right)-16 r^{2} g^{\prime}-32 r^{3}\left(2 g+r^{2} f\right) g\right] \\
& \left(r^{2}-s^{2}\right)\left[r\left(2 r+r^{2} L\right)\left(-8 g L\left(r^{2}-s^{2}\right)+8 r f\left(r^{2}-s^{2}\right)\right)+4 r L^{\prime}\left(s^{2}-r^{2}\right)+4 L\left(r^{2}-s^{2}\right)\right] \\
& -\left(r^{2}-s^{2}\right) 2 r L\left(4 r+2 s^{2} L\right)+2 r\left(2 r+r^{2} L\right)\left[2\left(2 r+r^{2} L\right)-\left(4 r+2 s^{2} L\right)\right],
\end{aligned}
$$

o que implica,

$$
\begin{aligned}
0= & L^{\prime}\left[8 r^{3} g-4 r\right]+L\left[8 r^{2}-8 r^{3} g^{\prime}-16 r^{4}\left(2 g+r^{2} f\right) g+8 r^{4} f-16 r^{2} g+4\right] \\
& +L^{2}\left[-8 r^{3} g+4 r\right]+16 r^{2} g\left[-16 r^{2} g^{\prime}-2 r\left(2 g+r^{2} f\right)+16 r^{3} f\right],
\end{aligned}
$$


para que, finalmente possamos obter a seguinte condição sobre $L(r)$,

$$
r a(r) L^{\prime}(r)-r a(r) L^{2}(r)+(r b(r)-a(r)) L(r)+2 b(r)=0,
$$

onde

$$
a(r):=2 r^{2} g(r)-1 \quad \text { e } \quad b(r):=-2 r^{2} g^{\prime}(r)-4 r^{3}\left(2 g(r)+r^{2} f(r)\right) g(r)+2 r^{3} f(r) .
$$

Por hipótese $a \neq 0$. Se considerarmos (4.20) como uma equação diferencial ordinária de $L(r)$, e observando que $-\frac{2}{r}$ é uma solução particular da equação de Ricatti (4.20), podemos obter a solução geral de (4.20):

$$
L(r)=-\frac{2}{r}-\frac{e^{-\int \frac{b(r)}{a(r)} d r}}{r^{3}\left(\int \frac{1}{r^{3}} e^{-\int \frac{b(r)}{a(r)} d r} d r+C\right)},
$$

onde $C \in \mathbb{R}$ é uma constante real.

Tendo em vista (2.40) temos que

$$
\varphi_{s}=\frac{2 s e^{\int 2 r\left(2 g+r^{2} f\right) d r}}{\left(r^{2}-s^{2}\right)^{2}} \varphi^{2} .
$$

Portanto, podemos reescrever (4.18) como,

$$
\frac{\eta_{s}(\varphi(r, s))}{\eta(\varphi)}=\left(\frac{2 s}{\left(r^{2}-s^{2}\right)^{2}}\right) \frac{r^{2}\left(r^{2}-s^{2}\right) e^{-\int \frac{b(r)}{a(r)} d r}}{4 r^{2} a(r)\left(r^{2}-s^{2}\right)\left(\int \frac{1}{r^{3}} e^{-\int \frac{b(r)}{a(r)} d r} d r+C\right)+2 s^{2} e^{-\int \frac{b(r)}{a(r)} d r} .}
$$

Por outro lado, com ajuda da seguinte identidade

$$
\int \frac{-2 s}{\left(r^{2}-s^{2}\right)\left(d(r)+l(r) s^{2}\right)} d s=\frac{1}{d(r)+l(r) r^{2}} \ln \left|\frac{r^{2}-s^{2}}{d(r)+l(r) s^{2}}\right|+T(r),
$$

podemos obter $\eta$ integrando em relação a $s$ a expressão anterior,

$$
\ln (\eta(\varphi(r, s)))=\frac{1}{2} \ln \left|\frac{4 r^{2} a(r)\left(r^{2}-s^{2}\right)\left(\int \frac{1}{r^{3}} e^{-\int \frac{b(r)}{a(r)} d r} d r+C\right)+2 s^{2} e^{-\int \frac{b(r)}{a(r)} d r}}{r^{2}-s^{2}}\right|+T(r) .
$$

Para simplificar a notação, considere $Z:=Z(r, s)$ sendo a expressão dentro do módulo da última igualdade, isto é,

$$
Z:=4 r^{2} a(r)\left(\int \frac{1}{r^{3}} e^{-\int \frac{b(r)}{a(r)} d r} d r+C\right)+\frac{2 s^{2}}{r^{2}-s^{2}} e^{-\int \frac{b(r)}{a(r)} d r},
$$

e suas respectivas derivadas (em $r$ e $s$ ),

$$
\begin{aligned}
& \frac{\partial Z}{\partial r}=\left(\int \frac{1}{r^{3}} e^{-\int \frac{b(r)}{a(r)} d r} d r\right)\left(4 r^{2} a(r)\right)^{\prime}+\left[\frac{4}{r} a(r)-\frac{4 r s^{2}}{\left(r^{2}-s^{2}\right)^{2}}-2 \frac{b(r)}{a(r)} \frac{s^{2}}{r^{2}-s^{2}}\right] e^{-\int \frac{b(r)}{a(r)} d r}, \\
& \frac{\partial Z}{\partial s}=\frac{4 s r^{2}}{r^{2}-s^{2}} e^{-\int \frac{b(r)}{a(r)} d r} .
\end{aligned}
$$


Podemos usar novamente o Lema 1.8.1 na equação (4.23), assim

$$
\begin{aligned}
{\left[1-\left(r^{2}-s^{2}\right)\left(2 g+s^{2} f\right)\right] r \frac{\partial Z}{\partial s}+s \frac{\partial Z}{\partial r} } \\
=s\left[\frac{4 r}{r^{2}-s^{2}}-4 r^{3} \frac{2 g+s^{2} f}{r^{2}-s^{2}}+\frac{4 a(r)}{r}+2 \frac{a^{\prime}(r)}{a(r)} \frac{s^{2}}{r^{2}-s^{2}}+\frac{\left.4 r\left(2 g+r^{2} f\right) s^{2}\right)}{r^{2}-s^{2}}\right] e^{-\int \frac{b(r)}{a(r)} d r} \\
+s\left(\int \frac{1}{r^{3}} e^{-\int \frac{b(r)}{a(r)} d r} d r\right)\left(8 r a(r)+4 r^{2} a^{\prime}(r)\right) \\
=s\left[\frac{4 r}{r^{2}-s^{2}}+4 r 2 g \frac{s^{2}-r^{2}}{r^{2}-s^{2}}+\frac{8 r^{2} g}{r}-\frac{4}{r}+2 \frac{a^{\prime}(r)}{a(r)} \frac{s^{2}}{r^{2}-s^{2}}\right] e^{-\int \frac{b(r)}{a(r)} d r} \\
+s\left(\int \frac{1}{r^{3}} e^{-\int \frac{b(r)}{a(r)} d r} d r\right)\left(8 r a(r)+4 r^{2} a^{\prime}(r)\right) \\
=s\left[\frac{4 s^{2}}{r\left(r^{2}-s^{2}\right)}+\frac{2 a^{\prime}(r)}{a(r)} \frac{s^{2}}{r^{2}-s^{2}}\right] e^{-\int \frac{b(r)}{a(r)} d r}+s\left(\int \frac{1}{r^{3}} e^{-\int \frac{b(r)}{a(r)} d r} d r\right)\left(8 r a(r)+4 r^{2} a^{\prime}(r)\right) \\
=s\left[\frac{2 s^{2}}{r^{2}-s^{2}} e^{-\int \frac{b(r)}{a(r)} d r}\left(2 a(r)+a^{\prime}(r)\right)+4 r^{2} a(r)\left(\int \frac{1}{r^{3}} e^{-\int \frac{b(r)}{a(r)} d r} d r\right)\left(2 a(r)+r a^{\prime}(r)\right] \frac{1}{r a(r)}\right. \\
=s \frac{2 a(r)+r a^{\prime}(r)}{r a(r)} Z .
\end{aligned}
$$

Voltando para (4.23), usamos a equação (1.73) para $2 \ln (\eta(\varphi))$,

$$
\begin{aligned}
0 & =\left[1-\left(r^{2}-s^{2}\right)\left(2 g+s^{2} f\right)\right] r \frac{\partial}{\partial s}(\ln |Z|+2 T(r))+s \frac{\partial}{\partial r}(\ln |Z|+2 T(r)) \\
& =\left[1-\left(r^{2}-s^{2}\right)\left(2 g+s^{2} f\right)\right] r \frac{1}{Z} \frac{\partial Z}{\partial s}+s \frac{1}{Z} \frac{\partial Z}{\partial r}+2 s T^{\prime}(r) \\
& =s \frac{2 a(r)+r a^{\prime}(r)}{r a(r)}+2 s T^{\prime}(r),
\end{aligned}
$$

obtendo, finalmente, a expressão de $T(r)$ :

$$
T(r)=\frac{1}{2} \int \frac{\left(r^{2} a(r)\right)_{r}}{r^{2} a(r)} d r=\frac{1}{2} \ln \left|r^{2} a(r)\right|+C_{1},
$$

onde $C_{1}$ é uma constante real.

Consequentemente, $\eta(\varphi)$ tem a seguinte forma:

$$
\eta(\varphi(r, s))=C_{2} \frac{\left|4 r^{2} a(r)\left(s^{2}-r^{2}\right)\left(\int \frac{1}{r^{3}} e^{-\int \frac{b(r)}{a(r)} d r} d r\right)-2 s^{2} e^{-\int \frac{b(r)}{a(r)} d r}\right|^{\frac{1}{2}}}{r \sqrt{\left|2 r^{2}-1\right|} \sqrt{r^{2}-s^{2}}},
$$

onde $C_{2}$ é uma constante real positiva.

Observamos que

$$
\begin{aligned}
\frac{b(r)}{a(r)} & =\frac{-s r^{2} g^{\prime}-2 r^{3} 4 g^{2}-4 r^{5} f g+2 r^{3} f-44 r g+4 r g}{2 r^{2} g-1} \\
& =-\frac{\left(2 r^{2} g-1\right)_{r}}{2 r^{2} g-1}-\frac{4 r g\left(2 r^{2} g-1\right)}{2 r^{2} g-1}-\frac{2 r^{3} f\left(2 r^{2} g-1\right)}{2 r^{2} g-1} \\
& =-\frac{a^{\prime}(r)}{a(r)}-2 r\left(2 g+r^{2} f\right) .
\end{aligned}
$$


Usando esta igualdade temos que

$$
\begin{aligned}
\left(\int \frac{1}{r^{3}} e^{-\int \frac{b(r)}{a(r)} d r} d r-\frac{e^{-\int \frac{b(r)}{a(r)} d r}}{2 r^{2} a(r)}\right)_{r} & \\
& =e^{-\int \frac{b(r)}{a(r)} d r}\left[\frac{1}{r^{3}}+\frac{b(r)}{a(r)} \frac{1}{2 r^{2} a(r)}+\frac{4 r a(r)+2 r^{2} a^{\prime}(r)}{4 r^{4} a^{2}(r)}\right] \\
& =e^{-\int \frac{b(r)}{a(r)} d r}\left[\frac{1}{r^{3}}-\frac{a^{\prime}(r)}{2 r^{2} a^{2}(r)}-\frac{2 r\left(2 g+r^{2} f\right)}{2 r^{2} a(r)}+\frac{a(r)}{r^{3} a^{2}(r)}+\frac{a^{\prime}(r)}{2 r^{2} a^{2}(r)}\right] \\
& =e^{-\int \frac{b(r)}{a(r)} d r}\left[\frac{1}{r^{3}}-\frac{2 g+r^{2} f}{r a(r)}+\frac{1}{r^{3} a(r)}\right] \\
& =e^{-\int \frac{b(r)}{a(r)} d r}\left[\frac{1}{r^{3}}-\frac{2 r^{2} g-1}{r^{3} a(r)}-\frac{1}{r^{3} a(r)}-\frac{r^{2} f}{r a(r)}+\frac{1}{r^{3} a(r)}\right] \\
& =e^{-\int \frac{b(r)}{a(r)} d r}\left[-\frac{r f}{a(r)}\right] .
\end{aligned}
$$

Portanto, obtemos a seguinte identidade

$$
\int \frac{1}{r^{3}} e^{-\int \frac{b}{a} d r} d r=\frac{e^{-\int \frac{b}{a} d r}}{2 r^{2} a}-\int \frac{r f}{a} e^{-\int \frac{b}{a} d r} d r
$$

Assim, usando (4.21) podemos melhorar a expressão de $\eta$ em (4.24) a menos de homotetia positiva:

$$
\begin{aligned}
& \eta(\varphi)=\frac{\left|4 r^{2} a(r)\left(s^{2}-r^{2}\right)\left(\int \frac{1}{r^{3}} e^{-\int \frac{b(r)}{a(r)} d r} d r\right)-2 s^{2} e^{-\int \frac{b(r)}{a(r)} d r}\right|^{\frac{1}{2}}}{r \sqrt{\left|2 r^{2}-1\right|} \sqrt{r^{2}-s^{2}}} \\
& =\frac{\left|s^{2}\left(-2 r^{2} a \int \frac{r f}{a} e^{-\int \frac{b}{a} d r} d r-2 r^{4} a \int \frac{1}{r^{3}} e^{-\int \frac{b}{a} d r} d r\right)\right|^{\frac{1}{2}}}{r \sqrt{\left|2 r^{2}-1\right|} \sqrt{r^{2}-s^{2}}} \\
& =\frac{\left|s^{2}\left(-2 r^{2}|a| \int r f e^{-\int 2 r\left(2 g+r^{2} f\right) d r} d r\right)-r^{2}\left(e^{-\int \frac{b}{a} d r}-2 r^{2} a \int \frac{r f}{a} e^{-\int \frac{b}{a} d r}\right)\right|^{\frac{1}{2}}}{r \sqrt{\left|2 r^{2}-1\right|} \sqrt{r^{2}-s^{2}}} \\
& =\frac{\left|\left(s^{2}-r^{2}\right)\left(e^{-\int \frac{b}{a} d r}-2 r^{2} a \int \frac{r f}{a} e^{-\int \frac{b}{a} d r} d r\right)-s^{2} e^{-\int \frac{b}{a} d r}\right|^{\frac{1}{2}}}{r \sqrt{\left|2 r^{2}-1\right|} \sqrt{r^{2}-s^{2}}} \\
& =\frac{\left|\left(s^{2}-r^{2}\right)\left(|a| e^{\int 2 r\left(2 g+r^{2} f\right) d r}-2 r^{2}|a| \int r f e^{\int 2 r\left(2 g+r^{2} f\right) d r} d r\right)-s^{2}\right| a\left|e^{\int 2 r\left(2 g+r^{2} f\right) d r}\right|^{\frac{1}{2}}}{r \sqrt{\left|2 r^{2}-1\right|} \sqrt{r^{2}-s^{2}}} \\
& =\frac{\left|\left(r^{2}-s^{2}\right) 2 r^{2} \int r f e^{\int 2 r\left(2 g+r^{2} f\right) d r} d r-\left(r^{2}-s^{2}\right) e^{\int 2 r\left(2 g+r^{2} f\right) d r}-s^{2} e^{\int 2 r\left(2 g+r^{2} f\right) d r}\right|^{\frac{1}{2}}}{r^{2} \sqrt{r^{2}-s^{2}}} \\
& =\frac{\mid\left(r^{2}-s^{2}\right) \int 2 r f e^{\int 2 r\left(2 g+r^{2} f\right) d r} d r-e^{\left.\int 2 r\left(2 g+r^{2} f\right) d r\right|^{\frac{1}{2}}}}{\sqrt{r^{2}-s^{2}}} .
\end{aligned}
$$


De (4.22), temos que $e^{\int L(r) d r}$, a menos de homotetia (positiva), pode ser escrito como

$$
e^{\int L(r) d r}=\frac{\sqrt{r^{2}-s^{2}}}{r^{2} \mid \int \frac{|a|}{r^{3}} e^{\int 2 r\left(2 g+r^{2} f\right) d r \mid}},
$$

e, finalmente obtemos a forma de $\phi(r, s)$, a menos de homotetia usando (4.14).

Observação 4.2.1. Observe que, se $g(r)=\frac{1}{2 r^{2}}$ e $L(r) \neq-\frac{2}{r}$, então $a(r)=b(r)=0$, e a identidade (4.20) é satisfeita trivialmente. Efetivamente o lado direito de (4.18) não depende de $L_{\phi}(r)$ e podemos reescrever (4.18) como,

$$
\frac{\eta_{s}(\varphi(r, s))}{\eta(\varphi(r, s))}=\frac{r^{2}}{s\left(r^{2}-s^{2}\right)}
$$

Integrando a última identidade em relação a $s$ temos:

$$
\eta(\varphi(r, s))=e^{c(r)} \frac{|s|}{\sqrt{r^{2}-s^{2}}}
$$

onde $c(r)$ é alguma constante como consequência da integração em $s$. A última identidade nos diz que $\phi$ não define uma métrica de Finsler.

Observação 4.2.2. Observe também que, se $g(r)=\frac{1}{2 r^{2}}$ e $L(r)=-\frac{2}{r}$, então (4.16) é satisfeita trivialmente, logo toda métrica de Finsler da forma $F(x, y)=|y| \phi(r, s)$, onde $\phi(r, s)$ é dada por:

$$
\begin{aligned}
\phi(r, s) & =e^{\int L(r) d r \sqrt{r^{2}-s^{2}} \eta(\varphi(r, s))} \\
& =\frac{\sqrt{r^{2}-s^{2}}}{r^{2}} \eta\left(\frac{r^{2}-s^{2}}{s^{2}} e^{-\int 2 r^{3} f(r) d r}\right),
\end{aligned}
$$

para alguma função diferenciável $\eta$, é do tipo Berwald.

O Lema 4.2.1, junto com as Observações 4.2.1 e 4.2.2 fornecem o seguinte Teorema:

Teorema 4.2.1. Sejam $f(r)$ e $g(r)$ funções diferenciáveis de $r \in I \subset \mathbb{R}$ tais que as condições (4.6) e (4.7) são satisfeitas. Suponha que $F(x, y)=|y| \phi(r, s)$ é uma métrica de Douglas com $S$-curvatura nula (isto é, $\phi>0$ satisfaz as equações (4.4) e (4.5)), então a menos de homotetia temos:

(i) Se $g \neq \frac{1}{2 r^{2}}$,

$$
F(x, y)=\frac{\sqrt{\left.\left|\left(|x|^{2}|y|^{2}-<x, y>^{2}\right) \int 2 r f e^{\int 2 r\left(2 g+r^{2} f\right) d r} d r-e^{\int 2 r\left(2 g+r^{2} f\right) d r}\right| y\right|^{2} \mid}}{\mid r^{2} \int 2 r f e^{\int 2 r\left(2 g+r^{2} f\right) d r} d r-e^{\int 2 r\left(2 g+r^{2} f\right) d r \mid}},
$$


(ii) Se $g=\frac{1}{2 r^{2}}$,

$$
F(x, y)=\frac{\sqrt{|x|^{2}|y|^{2}-<x, y>^{2}}}{|x|^{2}} \eta\left(\frac{|x|^{2}|y|^{2}-<x, y>^{2}}{<x, y>^{2}} e^{-\int 2 r^{3} f(r) d r}\right),
$$

onde $\eta$ é qualquer função tal que:

$$
\eta>\frac{2\left(r^{2}-s^{2}\right)}{s^{2}} e^{-\int 2 r^{3} f d r} \eta^{\prime}, \quad \text { quando } n \geq 2
$$

com a condição adicional

$$
-3 \sqrt{r^{2}-s^{2}} \eta^{\prime}+\frac{2 r^{2}}{s^{2}} e^{-\int 2 r^{3} f d r} \eta^{\prime \prime}>0, \quad \text { quando } n \geq 3 .
$$

Observação 4.2.3. No caso do elemento de volume de Holmes-Thompson, o caso (ii) no teorema anterior pode ser reescrito considerando a hipótese adicional:

$$
-\frac{2}{r}=\frac{2}{n+1}\left(\frac{A_{\phi}^{\prime}}{A_{\phi}}-r\left(\frac{1}{r^{2}}+r^{2} f\right)\right)=: L_{\phi}(r),
$$

isto é, as funções $\eta$ e $f$ têm que satisfazer a seguinte identidade:

$$
A_{\phi}(r)=\frac{c e \int r^{3} f d r}{r^{n}}
$$

onde $A_{\phi}(r)$ é o elemento de volume Holmes-Thompson dado na Proposição 1.6.1 e $c$ é uma constante positiva.

Observação 4.2.4. Note que se consideramos o elemento de volume Holmes-Thompson, a função $\phi(r, s)$ dada por (4.26) continua satisfazendo a equação (3.5), para alguma função $c_{1}(r)$, logo o Teorema 4.1 .3 continua válida se consideramos o elemento de volume Holmes-Thompson.

Observação 4.2.5. Exemplos (4.2.3) e (4.2.4) a seguir são do tipo (4.28).

Corolário 4.2.1. Seja $F=|y| \phi(r, s)$ uma métrica de Berwald do tipo (4.28), então o seu elemento de volume de Busemann-Hausdorff $A_{\phi}(r)$ a menos de homotetia é dada por

$$
A_{\phi}(r)=\frac{e^{\int r^{3} f d r}}{r^{n}} .
$$

Demonstração. Segue da substituição de $g=\frac{1}{2 r^{2}}$ e (4.28) em (4.7).

$$
\frac{A^{\prime}(r)}{A(r)}=r^{3} f(r)-\frac{n}{r}
$$

Logo, integrando em relação a $r$, temos o resultado.

Análogo ao Teorema 4.1.3, o Teorema 3.2.3 nos fornece o seguinte resultado. 
Teorema 4.2.2. Seja $F=|y| \phi(r, s)$ uma métrica de Finsler esfericamente simétrica sobre $M_{s}^{n}$. As seguintes afirmações são equivalentes:

a) F é do tipo Douglas com S-curvatura nula;

b) F é do tipo Berwald;

c) F é do tipo Landsberg.

Corolário 4.2.2. Seja $F=|y| \phi(r, s)$ uma métrica de Finsler esfericamente simétrica definida sobre $\mathbb{R}^{n}$, então $F$ é de Landsberg se, e somente se, F é uma métrica Riemanniana dado por (4.27).

Exemplo 4.2.1. Considerando $f(r)=g(r)=0$ no Teorema 4.2 .1 obtemos, a menos de homotetia, a seguinte métrica projetivamente plana com $S$-curvatura nula:

$$
F(x, y)=\frac{\sqrt{\left.\left|2 c_{1}\left(|x|^{2}|y|^{2}-<x, y>^{2}\right)-c_{2}\right| y\right|^{2} \mid}}{\left.\left|c_{2}\right| x\right|^{2}-c_{1} \mid},
$$

onde $c_{1}>0$ e $c_{2}$ são quaisquer constantes reais tal que

$$
2 c_{1}\left(|x|^{2}|y|^{2}-<x, y>^{2}\right)-c_{2}|y|^{2} \neq 0 \quad \text { e } \quad c_{2}|x|^{2}-c_{1} \neq 0 .
$$

Observação 4.2.6. Uma consequência interessante deste último exemplo é que sobre $\mathbb{R}^{n}$, $F(x, y)=|y| \phi\left(|x|, \frac{\langle x, y>}{|y|}\right)$ é uma métrica projetivamente flat com $S$-curvature nula se, e somente se, a menos de homotetia

$$
F(x, y)=|y|
$$

A partir da métrica de Douglas

$$
F(x, y)=<x, y>h(|x|)+c|y| e^{-\int_{0}^{|x|} 2 r g d r},
$$

onde $c$ é qualquer constante positiva e $h(r)$ tal que $F$ seja diferenciável e positiva para $y \neq 0$, podemos obter os seguintes exemplos:

Exemplo 4.2.2. Considerando $h(|x|)=0$, a seguinte métrica de Douglas

$$
F(x, y)=c|y| e^{-\int_{0}^{|x|} 2 r g d r}
$$

tem $S$-curvatura nula.

Exemplo 4.2.3. Considerando uma região anular $\mathbb{B}^{n}\left(\nu_{1}\right) \backslash \mathbb{B}^{n}\left(\nu_{2}\right),\left(\nu_{1}>\nu_{2} \geq 0\right)$ e $g(r)=$ $\frac{1}{2 r^{2}}$, a seguinte métrica de Douglas

$$
F(x, y)=c_{1} \frac{<x, y>}{|x|^{2}}+c \frac{|y|}{|x|}
$$

tem $S$-curvatura nula. Observe que este exemplo é do tipo (ii) do Teorema 4.2.1, e motiva a seguinte proposição, cuja prova é fornecida pela demonstração do Teorema 4.2.1. 
Proposição 4.2.3. Para quaisquer constantes $c_{1}$ e $c_{2}$, considere a família de funções definidas por

$$
\phi(r, s)=\left(c_{1} \frac{s}{r^{2}}+c_{2} \frac{1}{r}\right) \bar{\eta}\left(\varphi_{1}(r, s)\right),
$$

onde $\bar{\eta}$ é uma função arbitrária. Então ф satisfaz as equações (4.8) e (4.9).

Demonstração. Considerando $L(r)=-\frac{2}{r} g(r)=\frac{1}{2 r^{2}}$ e $f(r)=0$, na equação (4.13), as funções

$$
\varphi_{1}(r, s)=\frac{r}{\sqrt{r^{2}-s^{2}}} \quad \text { e } \quad \varphi_{2}(r, s)=\frac{s}{\sqrt{r^{2}-s^{2}}}
$$

são soluções de (4.13), portanto qualquer função $\eta$ de $\varphi_{1}$ ou $\varphi_{2}$ também é solução de (4.13). Para constantes $c_{1}$ e $c_{2}$, basta considerar

$$
\eta\left(\varphi_{1}, \varphi_{2}\right)=\left(c_{1} \varphi_{1}(r, s)+c_{2} \varphi_{2}(r, s)\right) \bar{\eta}\left(\varphi_{1}(r, s)\right)
$$

para obter a família de soluções $\phi(r, s)$ das equações (4.8) e (4.9) que estariam dadas por

$$
\phi(r, s)=e^{\int L(r) d r} \sqrt{r^{2}-s^{2}} \eta\left(\varphi_{1}, \varphi_{2}\right) .
$$

Isto conclui a demonstração.

As funções $\phi(r, s)$ obtidas em (4.32) são candidatas a definir uma métrica de Douglas $F(x, y)=|y| \phi(r, s)$ com $S$-curvatura nula, como por exemplo se considerarmos

$$
\eta(\varphi)=e^{\varphi^{-2}}+c
$$

onde $c$ é uma constante real. Assim obtemos o seguinte,

Exemplo 4.2.4. Considerando uma região anular $\mathbb{B}^{n}\left(\nu_{1}\right) \backslash \mathbb{B}^{n}\left(\nu_{2}\right),\left(\nu_{1}>\nu_{2}>0\right)$, a seguinte métrica de Douglas

$$
F(x, y)=\left(\frac{<x, y>}{|x|^{2}}+\frac{|y|}{|x|}\right)\left(e^{\frac{|x|^{2}|y|^{2}-<x, y>^{2}}{|x|^{2}|y|^{2}}}+3\right)
$$

tem $S$-curvatura nula.

Gostaria de deixar registrado aqui que, este último capítulo foi feito de forma independente e com técnicas diferentes do trabalho de L. Zhou [43] que aparece no arXiv em $20 / 10 / 2014$. 


\section{REFERÊNCIAS}

[1] Bácsó, S.; Chen, X.; Shen, Z. Curvature properties of $(\alpha, \beta)$-metrics. In: Sabau, S.; Shimada, H. (Ed.). Finsler Geometry, Sapporo 2005, In Memory Of Makoto Matsumoto. [S.1.]: Mathematical Society of Japan, 2007. v. 48, p. 73-110.

[2] Bácsó, S.; Matsumoto, M. On Finsler spaces of Douglas type. A generalization of the notion of Berwald space. Publ. Math. (Debr.), American Physical Society, v. 52, p. 385-406, Jan 1997.

[3] Bao, D. On two curvature-driven problems in Riemann-Finsler geometry. In: Advanced Studies in Pure Mathematics - Amsterdam and Tokyo; 48; 19-72 Symposium on Finsler Geometry 40th, Symposium on Finsler Geometry. [S.l.: s.n.], 2007.

[4] Benling, L. Projectively flat Matsumoto metric and its approximation. Acta Mathematica Scientia, v. 27, n. 4, p. 781 - 789, 2007. ISSN 0252-9602. Disponível em: http://www.sciencedirect.com/science/article/pii/S0252960207600757.

[5] Berwald, L. Untersuchung der Krümmung allemeiner metrischer Raume auf Grund des in ihnen Herrschenden Parallelismus. Math. Z., v. 25, p. 40-73, 1926.

[6] Berwald, L. Parallel übertragung in allgemeinen räumen. Atti Congr. Intern. Mat. bologna, v. 4, p. 263-270, 1928.

[7] Berwald, L. Über die n-dimensionalen geometrien konstanter krümmung in denen die geraden die kürzesten sind. Mathematische Zeitschrift, v. 30, p. 449-469, 1929. Disponível em: http://eudml.org/doc/168133.

[8] Cheng, X.; Shen, Z. On Douglas metrics. Publ. Math. (Debr.), v. 66, p. 503-512, 2007.

[9] Chen, X.; Mo, X.; Shen, Z. On the flag curvature of Finsler metrics of scalar curvature. Journal of the London Mathematical Society, v. 68, n. 3, p. 762-780, 2003. Disponível em: http://jlms . oxfordjournals .org/content/68/3/762.abstract.

[10] Chen, X.; Shen, Z. Randers metrics with special curvature properties. Osaka J. of Math., v. 40, p. 87-101, 2003.

[11] Chen, X.; Shen, Z. A class of Finsler metrics with isotropic S-curvature. Israel J. Math., v. 169, p. 317-340, 2009.

[12] Chen, X.; Shen, Z. Randers metrics of scalar flag curvature. J. Aust. Math. Soc., v. 87 , p. $359-370,2009$. 
[13] Chern, S.-S.; Shen, Z. Riemann-Finsler Geometry. [S.l.]: Springer Netherlands, 2005.

[14] Huang, L.; Mo, X. On spherically symmetric finsler metrics of scalar curvature. Journal of Geometry and Physics, v. 62, n. 11, p. 2279-2287, nov. 2012. ISSN 0393-0440. Disponível em: http://www.sciencedirect.com/science/article/ pii/S0393044012001398.

[15] Huang, L.; Mo, X. Projectively flat Finsler metrics with orthogonal invariance. Ann. Polon. Math., v. 107, p. 259-270, 2013.

[16] Ichijyō, Y. Finsler manifolds modeled on a Minkowski space. Journal of Mathematics of Kyoto University, Duke University Press, v. 16, n. 3, p. 639-652, 1976. Disponível em: http://projecteuclid.org/euclid.kjm/1250522876.

[17] Li, B.; Shen, Y.; Shen, Z. On a class of Douglas metrics. Studia Scientiarum Mathematicarum Hungarica, v. 46, n. 3, p. 355-365, set. 2009. Disponível em: http://dx.doi.org/10.1556/SScMath.2009.1096.

[18] Matsumoto, M. Finsler spaces with $(\alpha, \beta)$-metric of Douglas type. Tensor., The Tensor Society., Chigasaki, Japan [etc., v. 60, n. 2, p. 123-134, 1998. ISSN 0040-3504.

[19] Matsumoto, M. On C- reducible Finsler spaces. Tensor N.S., v. 24, p. 29-37, 1972.

[20] Matsumoto, M. On Finsler spaces with Randers metric and special forms of important tensors. Duke University Press, p. 477-498, 1974. ISSN 0023-608X. Disponível em: http://projecteuclid.org/euclid.kjm/1250523171.

[21] Matveev, V. S. On "all regular landsberg metrics are always berwald" by Z. I. Szabó. Balkan Journal of Geometry and its Applications (BJGA), Balkan Society of Geometers, Bucharest; Geometry Balkan Press, v. 14, n. 2, p. 50-52, 2009. Disponível em: http: //eudml .org/doc/224357.

[22] Mo, X.; Yang, C. The explicit construction of Finsler metrics with special curvature properties. Differential Geometry and its Applications, v. 24, n. 2, p. 119-129, mar. 2006. ISSN 0926-2245. Disponível em: http://www.sciencedirect.com/science/ article/pii/S0926224505000793.

[23] Mo, X.; Solórzano N.M. and Tenenblat K. On spherically symmetric Finsler metrics with vanishing Douglas curvature Differential Geom. Appl., v. 31 no. 62013.

[24] Mo, X.; Zhou, L. The curvatures of spherically symmetric Finsler metrics in $\mathbf{R}^{n}$. ArXiv e-prints, fev. 2012.

[25] Okada T. On models of projectively flat Finsler spaces of constant negative curvature. Tensor, N.S. v. 40, p. 117-123, 1983. 
[26] Randers, G. On an asymmetrical metric in the four-space of general relativity. Phys. Rev., American Physical Society, v. 59, p. 195-199, Jan 1941. Disponível em: http: //link.aps.org/doi/10.1103/PhysRev. 59.195.

[27] S.F. Rutz, Symmetry in Finsler spaces, Contemp. Math. 196 (1996) 289-300.

[28] Sevim, E. S.; Shen, Z.; Zhao, L. On a class of Ricci-flat Douglas metrics. Int. J. Math., World Scientific Publishing Co., v. 23, n. 06, p. 1250046-, maio 2012. ISSN 0129-167X. Disponível em: http://dx.doi.org/10.1142/S0129167X12500462.

[29] Shen, Z. Curvature, distance and volume in Finsler geometry. Bures-sur-Yvette, 1997.

[30] Shen, Z. Volume comparison and its applications in riemann-finsler geometry. Advances in Mathematics, v. 128, n. 2, p. 306-328, jun. 1997. ISSN 0001-8708. Disponível em: http://www. sciencedirect.com/science/article/pii/S0001870897916303.

[31] Shen, Z. Differential Geometry of Spray and Finsler Spaces. [S.1.]: Springer Netherlands, 2001.

[32] Shen, Z. Lectures on Finsler Geometry. [S.1.]: World Scientific, 2001.

[33] Shen, Z. On a class of Landsberg metrics in Finsler geometry. Canad. J. Math., v. 61 no. 6, p. 1357-1374, 2009.

[34] Shen, Z. Projectively flat Finsler metrics of constant flag curvature. Trans. Am. Math. Soc., v. 355, p. 1713-1728, 2003.

[35] Szabó, Z. All regular Landsberg metrics are Berwald. Springer Netherlands, v. 34, n. 4, p. 381-386, 2008. ISSN 0232-704X. Disponível em: http://dx.doi.org/10.1007/ s10455-008-9115-y.

[36] Szabó, Z. Correction to "all regular Landsberg metrics are Berwald"'. Springer Netherlands, v. 35, n. 3, p. 227-230-, 2009. ISSN 0232-704X. Disponível em: http://dx.doi.org/10.1007/s10455-008-9131-y.

[37] Szabó, Z. I. Positive definite Berwald spaces (structure theorems). Tensor N.S., v. 35, p. 2539, 1981.

[38] Tian, Y.; Cheng, X. Ricci-flat Douglas metrics $(\alpha, \beta)$-metrics. Differential Geometry and its Applications, v. 30, n. 1, p. 20-32, fev. 2012. ISSN 0926-2245. Disponível em: http://www.sciencedirect.com/science/article/pii/S0926224511001616.

[39] Xin, H. The properties meaning of Randers metrics with isotropic S-curvature. Adv. in Math. (Chinese), v. 34, p. 717-730, 2005. 
[40] Yu, C.; Zhu, H. On a new class of Finsler metrics. Differential Geometry and its Applications, v. 29, n. 2, p. 244-254, mar. 2011. ISSN 0926-2245. Disponível em: http://wWw. sciencedirect.com/science/article/pii/S0926224510000823.

[41] Yu, Y.-y.; You, Y. Projectively flat exponentialFfinsler metric. Journal of Zhejiang University SCIENCE A, Zhejiang University Press, v. 7, n. 6, p. 1068-1076, 2006. ISSN 1009-3095. Disponível em: http://dx.doi .org/10.1631/jzus.2006. A1068.

[42] Zhou, L. Spherically symmetric Finsler metrics in $\mathbb{R}^{n}$. Publ. Math. Debrecen, v. 80, n. 1-2, p. 67-77, 2012.

[43] Zhou, L. The spherically symmetric Finsler metrics with isotropic S-curvature. arXiv:1409.2587 